\title{
Tailoring growth and local composition by oblique-incidence deposition: a review and new experimental data
}

\author{
Herma van Kranenburg and Cock Lodder \\ MESA Research Institute, University of Twente, P.O. Box 217, 7500 AE Enschede (Netherlands)
}

(Received March 8, 1993; accepted September 24, 1993)

\begin{abstract}
In this paper features are discussed that are related to the nucleation and growth of thin films deposited by physical vapour deposition. Although the paper is mainly focused on oblique-incidence deposition, a normally-impinging vapour flux will also be described. Two cases of oblique-incidence deposition will be discussed: one obliquely-impinging flux and two obliquely-impinging fluxes from opposite directions. With respect to the microstructure, both similarities and differences between these depositions will be described. Further, a special feature of two obliquely-impinging vapour fluxes is dealt with, which is the local composition in the lateral direction (parallel to the substrate). The oblique and opposing directions can induce an inhomogeneous chemical composition. This process is referred to as process-induced compositional separation. In addition to reviewing the literature, the paper summarizes experimental data on obliquely co-evaporated $\mathrm{Co}-\mathrm{Cr}, \mathrm{Co}-\mathrm{Ag}$ and Co-Ta films. It will be shown from these experimental data that the two vapour directions can be exploited to tailor compositional inhomogeneities.
\end{abstract}

\section{Introduction}

Thin films are generally made by deposition techniques such as physical vapour deposition (i.e. sputtering or evaporation), chemical vapour deposition, electroless deposition, electrodeposition, or indirect methods, for instance thermic growth and doping techniques. In the indirect methods a reaction with the already existing layer or substrate occurs: for example, in thermic growth processes, the substrate is heated in a gaseous environment, like oxygen, by which the oxide layer of the substrate material is formed. In doping techniques the dopant atoms are ionized, accelerated to high energies by an electric field and, after mass-separation of the unwanted ion species, focused on the (semiconductor) target, where they penetrate the surface. Electroless deposition and electrodeposition are based on chemical principles and can generally be carried out at room temperature and atmospheric pressure. In chemical vapour deposition (CVD) the desired film material, in the form of a vapour, is led along the substrate, mostly with the help of a carrier gas. At the intersection with the substrate/film surface, a chemical reaction takes place between the desired material and the substrate, so that a solid layer is formed. This process usually takes place under atmospheric pressure and a relatively high temperature. Physical vapour deposition (PVD) is carried out under (high) vacuum conditions (and is a non-equilibrium process). In sputtering, ions are accelerated towards the target, which consists of the material to be deposited. The ions can arise from a plasma between the substrate and target or from a special ion source (ion-beam deposition). The ion bombardment of the target knocks out (by impulse transfer phenomena) high-energy (clusters of) atoms that can condense on the substrate. In evaporation the desired film material is situated in a source that is heated 
above the material's melting or sublimation temperature. As a result the material evaporates or sublimates and thus a vapour flux arises. The substrate is placed in the vapour flux, and by condensation of the vapour a film will grow.

Each deposition technique has special characteristics which are reflected in the nucleation and growth of films. For instance, we have already mentioned the vacuum and atmospheric surroundings in physical and chemical vapour depositions, respectively. In this paper we shall only deal with physical vapour deposition (mostly evaporation). After the introduction in this section, nucleation will be reviewed (Section 2), followed by the growth of thin films deposited under normal incidence (Section 3). Then in Section 4 growth under obliqueincidence vapour flux is discussed. Here will be described how the shadowing and adatom mobility can be tailored. In addition, examples from experiments will be shown. In the next section, Section 5, the growth of films deposited under co-oblique vapour flux directions is discussed. As for the deposition method, in Section 5 we deal with evaporation. The reason is that our choice of deposition method was made in favour of evaporation, and in Section 5 we shall focus on our results. Besides the morphology on a macro scale, the micro scale of the distribution of the materials (compositional separation) is included in the discussion. Here too, a number of examples are given. These mainly concern films for magnetic recording applications. A point we would like to mention is that the major part of Sections $2-5$ is based on the Ph.D. work of the first author and, as such, published in ref. 1. Finally in Section 6 conclusions are drawn.

In the remaining part of this introduction we shall mention some features of obliqueincidence deposition. With respect to previous papers [2-6], new additional experimental data on $\mathrm{Co}-\mathrm{Cr}$ samples are presented in this paper. Further, the data on $\mathrm{Co}-\mathrm{Ag}$ and $\mathrm{Co}-$ Ta are new. Besides this, the additional value of the present paper is the overall interpretation and correlation of the numerous data.

In oblique-incidence deposition the incoming vapour flux makes an angle $\alpha_{i}$ with the film normal, see Fig. 1. A specific feature in such a deposition is that certain parts of the growing film are shadowed for direct impingement (the so-called shadowing effect). As a result, oblique-incidence deposition generates anisotropic characteristics in thin films. This applies to anisotropy in optical [7-10], magnetic $[9,11,12]$ and electrical $[12,13]$ properties. In magnetic recording this is mostly exploited for the magnetically "easy" direction, see for example ref. 14 .

In Fig. 2 an example is given for the dependence of $K_{\mathrm{u}}$ on the incidence angle for $\mathrm{Ni}-\mathrm{Fe}$ [12]. Here $K_{\mathrm{u}}$, the uniaxial magnetic anisotropy constant, indicates the preferential direction of the magnetization (the so-called easy axis). With $K_{u}$ positive, the easy axis is

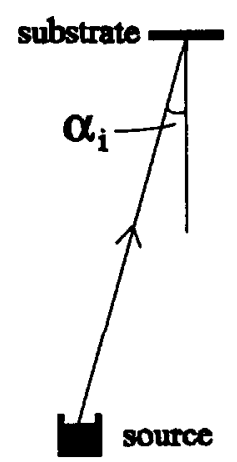

Fig. 1. Oblique-incidence evaporation from one source. 


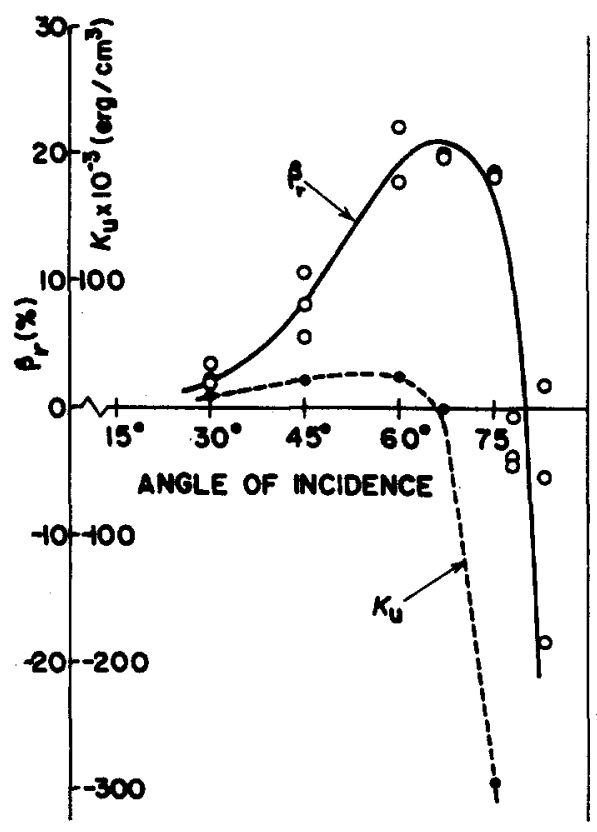

Fig. 2. Dependence of the uniaxial magnetic anisotropy constant $K_{\mathrm{u}}$ and the resistivity anisotropy constant $\beta_{\mathrm{r}}$ on the incidence angle in thin films of $84.5 \% \mathrm{Ni}-\mathrm{Fe}[12]$.

perpendicular to the incidence plane, whereas with $K_{\mathrm{u}}$ negative, it is parallel. Around $65^{\circ}$, $K_{\mathrm{u}}$ switches its sign. This switch is observed in many oblique-incidence deposited materials, mostly around $\alpha_{i}=60-80^{\circ}$. Its origin, being morphological, will be discussed more extensively in this paper. In applied optics, for example, the birefringence of obliquely deposited films can be used [15]. An absorptional property of the oblique columnar structure can result in polarization-dependent and angular-selective transmittance values [16].

As a third example, the electrical resistivity is taken. An anisotropy in structural defects can give rise to an anisotropy in the resistance associated with the imperfection scattering of the conduction electrons. Figure 2 shows the dependence of $\beta_{\mathrm{r}}$ on the angle of incidence [12]. Here $\beta_{\mathrm{r}}$ is the resistivity anisotropy constant equal to $\left(\rho_{\|}-\rho_{\perp}\right) /\left(\rho_{\|}+\rho_{\perp}\right)$ where $\rho_{\|}$and $\rho_{\perp}$ are the resistivities when the current is, respectively, parallel and perpendicular to the plane of incidence. As can be seen in Fig. 2, the dependencies of $\beta_{\mathrm{r}}$ and $K_{\mathrm{u}}$ on the incidence angle are similar. Thus analogy exists for the magnetic and electrical cases. The same holds for the magnetic and optical cases: see Fig. 3, which shows a combined figure of the differential absorptional constant and magnetic anisotropy as a function of the incidence angle for $\mathrm{Ni}-\mathrm{Fe}$ films [9]. The choice of material influences the anisotropic behaviour considerably. For instance, in obliquely evaporated $\mathrm{Cu}$ the dependence of $\beta_{\mathrm{r}}$ on the incidence angle is considerably different from that of Ni-Fe films, compare Figs. 2 and 4.

Summarizing, it can be said that the preparation technique itself is relevant for the material produced, and oblique deposition offers a possibility to create anisotropic behaviour. Such anisotropic behaviour is attributable to the anisotropic film microstructures that are formed due to the shadowing mechanism. Its combination with limited adatom mobility during growth allows different film packing densities and morphologies to arise in the directions parallel and perpendicular to the incidence plane. Factors contributing to the nucleation and growth processes will be reviewed and discussed in this paper. In the second half of the paper we will focus on obliquely co-evaporated films. It will be shown that, 


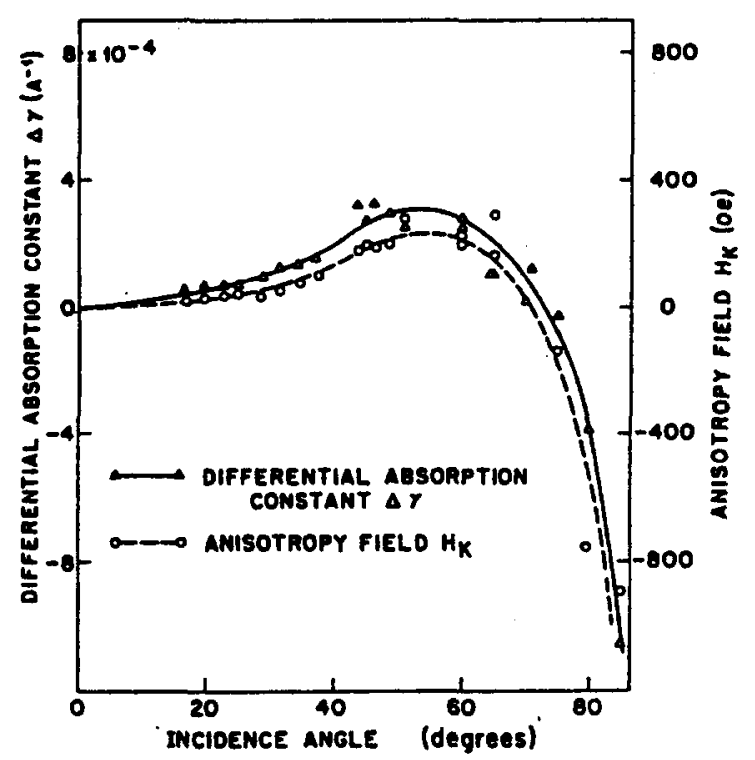

Fig. 3. Dependence of the differential absorption coefficient and anisotropy field on the incidence angle in obliquelyevaporated thin films of $83 \% \mathrm{Ni}-\mathrm{Fe}\left(T_{\mathrm{s}}=200^{\circ} \mathrm{C}\right)$ [9].

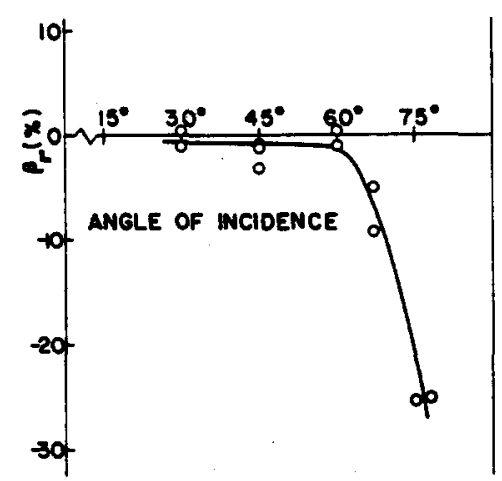

Fig. 4. Dependence of the resistivity anisotropy constant $\beta_{\mathrm{r}}$ on the incidence angle in thin films of $\mathrm{Cu}$ [12].

besides the possibility to tailor the morphology, oblique co-evaporation provides an additional tool to tailor the local composition.

Both the evaporation and sputtering processes can be used to induce anisotropic behaviour by oblique-incidence deposition. Since the degree of shadowing strongly depends on the angle of incidence, the mean free path of the incoming atoms is important. For instance, in sputter deposition, where usually a high residual gas pressure is used (around $1 \mathrm{~Pa}$ ), the mean free path of the sputtered atoms is very short and the atoms are spatially scattered. This results in a large distribution of incidence angles. Consequently, the anisotropy induced by the morphological differences is small; in fact smaller than in evaporation processes. However, even with sputtering, if a dominant atom direction of incidence is present then an anisotropy can be induced.

Compared with sputtering, where high-energy particles interact with the film surface, the growth mechanism can be more easily understood in evaporated films. The kinetic energy of the particles once they are near the film surface is, in practical situations, about ten times higher in sputtering than in evaporation [17]. As just explained, the absence of 
a plasma near the substrate allows better-directed vapour fluxes, with a small spread of incidence angles. Therefore, evaporation or ion-beam sputter deposition (where the plasma is not near the substrate) is more suitable for oblique deposition than the glow- discharge sputtering methods.

\section{Nucleation of thin films}

The overall process of thin-film formation can be roughly divided into the following stages: nucleation, island growth and coalescence stage, channel-filling stage, and growth of continuous films (see, for example, refs. 18 and 19). These stages will be described in a phenomenological way and are schematicaly shown in Fig. 5 [20]. The growth of the continuous films deposited by evaporation and sputtering will be described more extensively; first for normal incidence in Section 3, followed by oblique incidence in Sections 4 and 5 .

Nucleation starts with the impingement of a vapour particle (atom) on the substrate. The particle can either be reflected from the substrate or be (physically) adsorbed. A physically adsorbed particle (now called adatom) can diffuse over the substrate and then re-evaporate (desorption) or it can be pinned at a location where its energy is at a local minimum. More incoming particles reaching the substrate behave in a similar way. If two or more particles form a cluster (nucleate), the possibilities for migration or re-evaporation from the substrate become smaller. At a certain size of cluster, the position is fixed and the layer will grow from that cluster. During deposition several nuclei are formed. The position of the nucleation centres can be randomly distributed or according to the distribution of the energetic minima present owing to, for example, a crystalline structure of the substrate or a combination of surface roughness and oblique-incidence deposition.
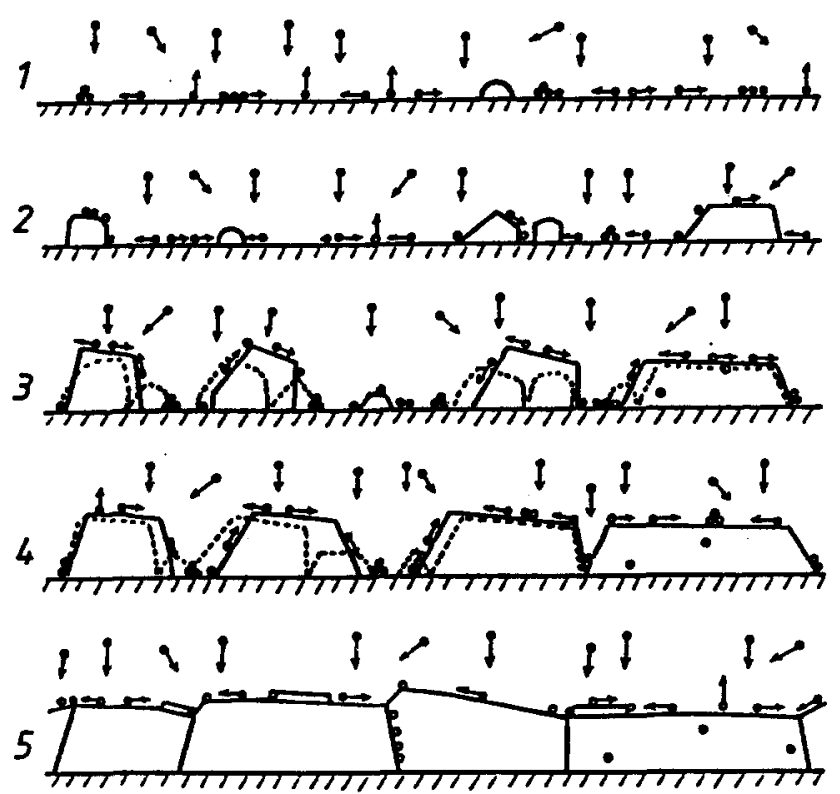

Fig. 5. Stages of structure evolution in polycrystalline thin films: (1) nucleation, (2) crystal (grain) growth, (3) coalescence, (4) filling the channels and (5) growth of continuous film. Crystals before coalescence are marked by broken lines; solid circles mark adatoms and open circles impurity species [20]. 
Critical sizes of nuclei are determined by the competition between their surface and volume free energy. First of all, because small particles display a higher vapour pressure than the bulk material under the same conditions, for condensation to occur a supersaturation ratio larger than unity is required. Thus the partial pressure of the film material in the gas phase must be larger than its vapour pressure in the condensed phase at that temperature. Below a certain critical size a nucleus is not stable, because of its large surface-to-volume ratio. With higher supersaturation the size of the critical nucleus decreases and nucleation takes place at more sites [21].

The nuclei grow by acquiring (mobile) adatoms. As they grow larger, they will merge into larger clusters, called islands. This is accompanied by a decrease of the area occupied by the separate nuclei. The surface energy is thus lowered, which can be experimentally observed as a decrease of stress [22]. The film at this stage is structurally discontinuous. With increasing adatom mobility, the density of the nuclei decreases and correspondingly the size and the separation of the nuclei increases. In ref. 21 , for example, it was shown by simulation that with increasing surface diffusion the (area of the) nuclei becomes larger and the surface smoother.

As the islands grow large enough to touch each other, coalescence takes place at the interface between them, hereby minimizing the surface free energy. With increasing size, a complete change of shape upon coalescence is less likely to occur. The main changes in shape occur near the junction between islands. The result is a network structure consisting of channels covered with material deposited later.

The channels are filled up during further deposition due to, for instance, secondary nucleation and volume diffusion. If the adatom mobility is high enough, the channels will disappear and a continuous film is formed. If not, a large concentration of defects, primarily vacancies or microscopic voids, is frozen in. The larger the adatom mobility, the smaller the number of frozen-in defects will be. A small adatom mobility also implies more disorder or limited short-range order in the islands or clusters of atoms.

Growth of the continuous film starts by the covering of the network structure of channels (which are filled to some degree) with material deposited later. It continues by subsequent deposition of material. The mode of thin-film growth is essentially a kinetic phenomenon depending on deposition conditions like temperature and deposition rate [18]. It was proposed that different growth modes like 3-D island growth (Volmer-Weber), layer-by-layer growth (Frank van der Merwe), and an intermediate mode, where first a continuous layer forms on the substrate before island growth becomes highly advanced (Stranski-Krastanov), could occur depending on the interrelation of the surface free energy of the substrate, the deposit and the substrate-deposit interface. However, depending on the kinetics, transitions between these modes are possible [18]. The eventual development of the continuous film of course, depends on the first nuclei formed and the growth mode. The layer-by-layer growth is essentially epitaxial and is not encountered in the films investigated here. Instead a porous columnar morphology develops. In Section 4 a description will be given of how the oblique deposition promotes such a morphology. First, in Section 3 a structure zone model for evaporated and sputtered thin films under normal incidence is reviewed, which describes columnar growth in a certain deposition region.

\section{Growth of evaporated and sputtered thin films: structure zone models}

The morphology (size and shape of the crystallites) and the texture (preferential orientation of the crystallites) are the two most important properties for describing the 
microstructure of the film. The morphology is strongly dependent on the deposition parameters such as the substrate temperature $T_{\mathrm{s}}$. We will first describe two well-known models, namely those of Movchan and Demchishin [23] and Thornton [24,25], which are both based on experimental observations. These models are often used as guidelines for the morphology. However, great care must be taken in doing so when thin films are considered. That is to say, these two models relate to relatively thick films (more than $25 \mu \mathrm{m}$ ) and therefore cannot be applied straightforwardly to thin films of, for example, the order of several hundreds of nanometres. In this section the description of these models is followed by several extensions (e.g. to thinner film thicknesses) and other features that play a role in the growth mechanism.

Movchan and Demchishin introduced a model for evaporated metals and oxides in which the dependence of the morphology on the ratios of $T_{\mathrm{s}}$ and $T_{\mathrm{m}}$ is shown. $\left(T_{\mathrm{m}}\right.$ is the melting temperature of the material used.) The model has been extended to include Ar sputtering conditions by Thornton, who added the pressure dependence of the morphology. In the model of Movchan and Demchishin three zones can be discerned (see Fig. 6(a)), of which a short description will be given. The transition between zones 1 and 2 appears at a ratio of $T_{\mathrm{s}}$ to $T_{\mathrm{m}}$ of 0.3 for metals and $0.22-0.26$ for oxides, and that between zones 2 and 3 at $0.45-0.5$. In Thornton's model an intermediate zone $T$ is present that lies between zones 1 and 2, see Fig. 6(b). In the review given below it should be kept in mind that zone $T$ is only defined for Thornton's model.

Zone 1. The first zone displays a porous, tapered columnar structure. The column boundaries are not very clear. Owing to the low ratio between $T_{\mathrm{s}}$ and $T_{\mathrm{m}}$, the surface and volume diffusions are small. The structure is determined by collision of growing islands and shadowing where parts of the substrate or film are shielded against direct impingement of particles because of the roughness of the substrate or film. The film surface is quite rough.

Zone $T$. This zone consists of a dense array of poorly defined fibrous grains. Compared to zone 1 , the surface mobility is larger, the shadowing effect is smaller, the density of the films is larger and the film surface is smoother.

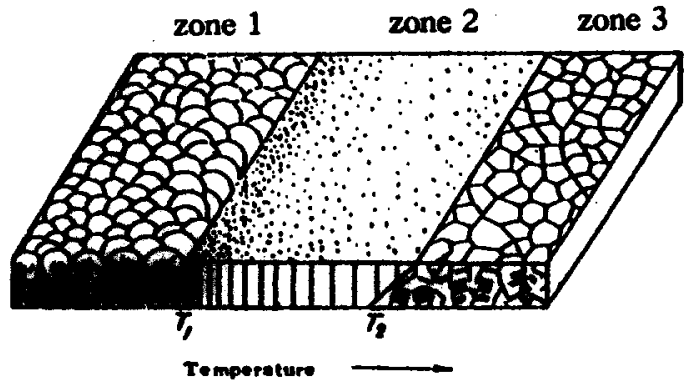

(a)

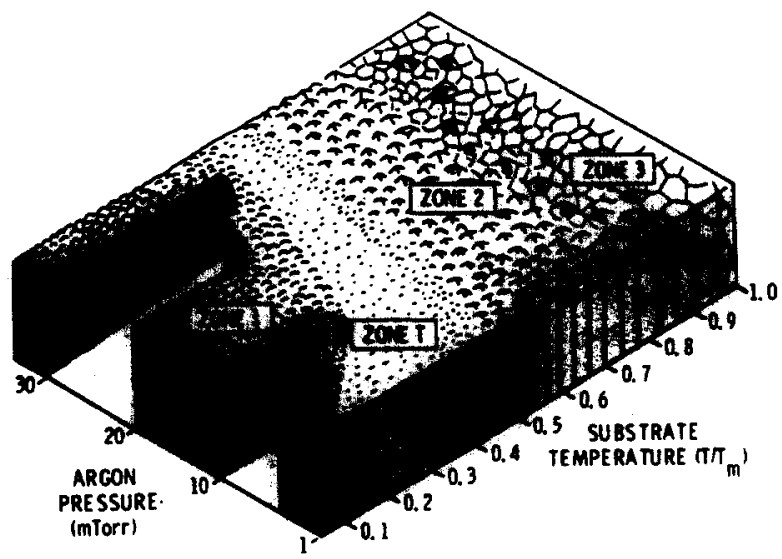

(b)

Fig. 6. Structure zone models: (a) evaporated films [23]; (b) sputtered films [25]. 
Zone 2. The substrate temperatures in zone 2 are high enough to let surface diffusion govern the formation of the layer. The greater adatom mobility decreases the effect of shadowing, and the density of the films becomes larger. Because of the greater mobility, the particles are able to spread more uniformly over the surface. Besides surface diffusion, migration of crystallite boundaries can be responsible for the crystal growth [26]. The resulting structure is straight columnar with well-defined column boundaries. The columns grow instantly from the substrate and are present up to the film surface. The surface is smooth and matt.

Zone 3. In this case the bulk diffusion is dominant owing to the high temperatures. Further recrystallization can occur [26]. The crystallites are rather large and their boundaries are randomly oriented with respect to the substrate. The surface is very smooth and bright.

The Movchan-Demchishin and Thornton models were originally developed for very thick films (300-2000 $\mu \mathrm{m}$ and 25-250 $\mu \mathrm{m}$ respectively) deposited at a high rate (30-300 $\mathrm{nm} \mathrm{s}{ }^{-1}$ and $0.08-333 \mathrm{~nm} \mathrm{~s}^{-1}$ respectively). Later they were improved by Messier et al. [27] for the regions with ratios of $T_{\mathrm{s}}$ to $T_{\mathrm{m}}$ below approximately 0.5 in order to accommodate both the evolution of morphology with increasing film thickness and the separate thermal and bombardment-induced (as, for example, in sputtering) mobility. (Note: the low $T_{\mathrm{s}} / T_{\mathrm{m}}$ region is of special interest in device fabrication because of the importance of the refractory coating materials and of reducing processing temperatures.) Film thicknesses of $10 \mathrm{~nm}$ up to $30 \mu \mathrm{m}$ were investigated. It was found that these films deposited under conditions of low adatom mobility were characterized by columnar structures with a wide range of systematically varying column and void sizes. The evolution of each structural level was not linear with the film thickness but appeared to have a logarithmic-linear dependence. Characteristic columnar sizes for film thicknesses around $100-200 \mathrm{~nm}$ ranged from $20-40$ $\mathrm{nm}$ for the lowest thermal-induced adatom mobility $\left(T_{\mathrm{s}} / T_{\mathrm{m}}=0\right)$ to approximately $20 \mathrm{~nm}$ for the highest $\left(T_{\mathrm{s}} / T_{\mathrm{m}}=0.5\right)$. This decrease in columnar size is probably due to a change from tapered columns to straighter ones. Figure 7 [27] is a schematic diagram showing the change

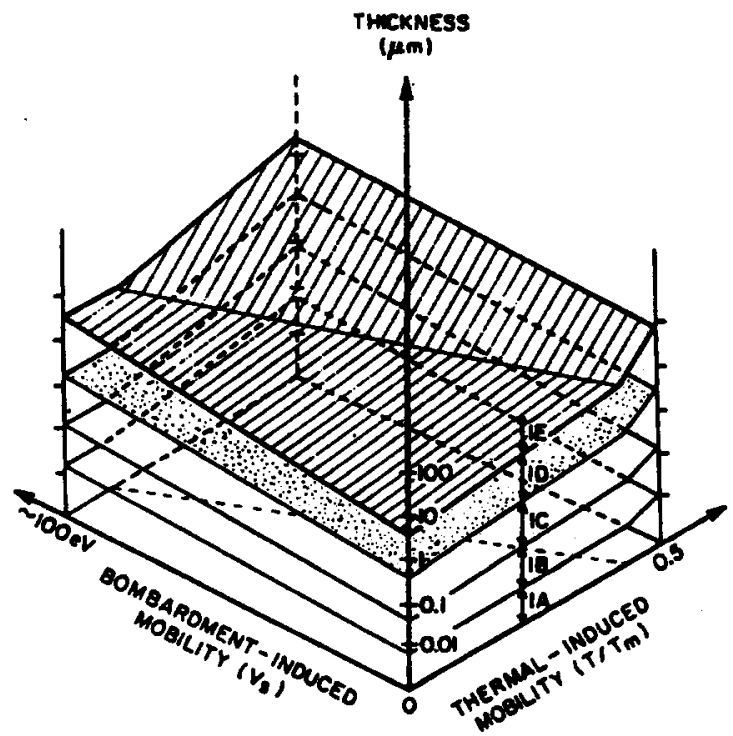

Fig. 7. Revised structure zone model for physical structures [27]. 
in columnar size with bombardment-induced mobility (measured by the substrate potential $V_{\mathrm{s}}$ ) and thermal-induced mobility $\left(T_{\mathrm{s}} / T_{\mathrm{m}}\right)$. In the diagram of Fig. 7 the thickness scale is logarithmic and the labels IA, IB, ..., IE correspond to five characteristic columnar sizes of 1-2, 5-20, 20-40, 50-200 and 200-400 nm.

Grovenor et al. [26] investigated the granular size for a number of evaporated metals with film thickness of $100 \mathrm{~nm}$ as a function of $T_{\mathrm{m}} / T_{\mathrm{s}}$, see Fig. 8(a). It was found that the activated processes that control the granular growth scale with $T_{\mathrm{m}}$. This is symbolized in the diagram of Fig. 8(b). The concept of athermal crystallization is proposed to account for the crystallinity of the films for the low-temperature region (zone 1). Small groups of atoms are assumed to crystallize athermally, by which the heat created by crystallization is released, which further stimulates the crystallization until neighbouring nuclei impinge. The resulting crystallite size is then governed by the density of the crystalline nuclei. In the case of most metals the precursor to crystalline transformation involves a volume decrease. Since such a volume decrease is constrained by the substrate, the existence of a tensile stress in films grown at a low ratio of $T_{\mathrm{s}}$ to $T_{\mathrm{m}}$ is consistent with the above concept of athermal crystallization [26]. Experimental high-resolution TEM observations on magnetronsputtered Co-Cr [28] confirm the concept of ref. 26.

The transition from zone 1 structures through zone $T$ to those of zone 2 occurs because different grain boundaries become mobile at different temperatures. In zone 1 almost all the boundaries are immobile (regime (a)), whilst in zone 2 all are mobile (regime (c)). In zone $\mathrm{T}$ an increasing number of the boundaries present become mobile. Consequently, the probability of any boundary moving across grains and reacting to form another mobile boundary is increased. Since larger grains have more neighbours, continued growth of particular grains, once they have established a size advantage, is preferable (regime (b)). The onset of surface diffusion in zone $T$ contributes to the decrease in porosity. In zone 2 all boundaries are mobile, and columnar structures with uniform grain diameter develop. In this regime (c), grain growth occurs together with granular epitaxy. For both mechanisms the activation energies (i.e. for surface diffusion and for grain-boundary migration) are similar. In zone 3, more lateral grain growth occurs and larger grains develop. In addition, it is expected that deposition of an alloy film will result in a finer grain size than that seen in a pure metal film deposited under the same conditions. The presence of a solute in the

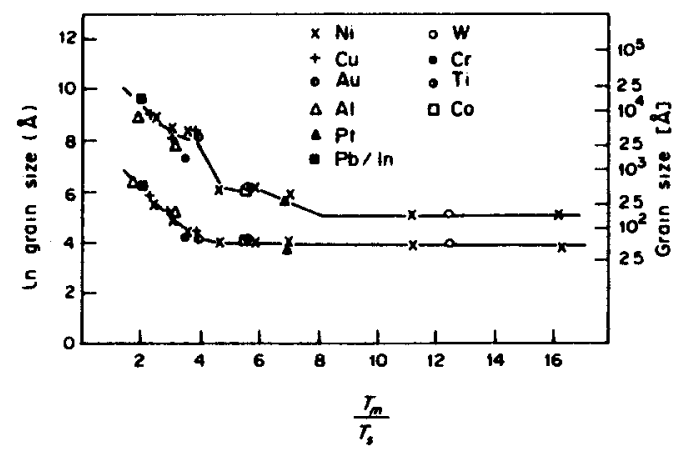

(a)

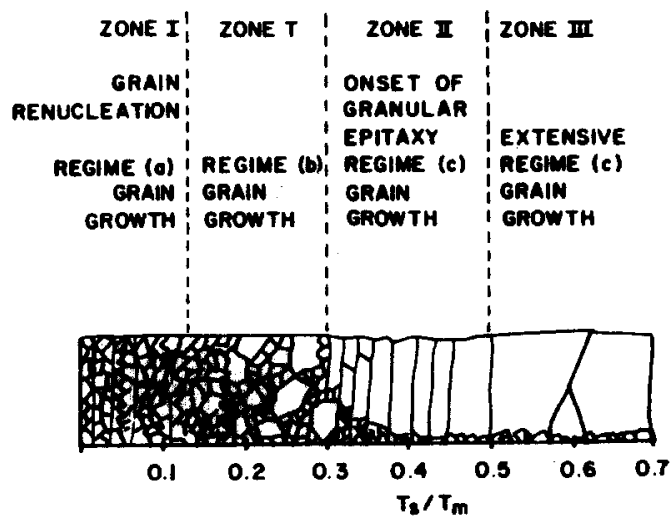

(b)

Fig. 8. (a) Plot of grain-size variation with substrate temperature for thin films of ten different metals [26]. (b) Zone model for the grain structure of vapour-deposited metal films, including the mechanisms that are proposed to control the grain structures [26]. 
deposit will limit the grain growth by reducing the number of boundaries that are mobile at any $T_{\mathrm{s}}$.

The structure zone models are found to be applicable to a wide range of materials. Reports on several kinds of films have been made that satisfy the structure zone model described above. Alloys are included, as are pure films, crystalline metallic films, amorphousand crystalline-ceramic films and amorphous semiconductor films [27,29,30].

Common features in thin-film morphology, particularly the similarity in shape and form which persisted over a large range of film thicknesses and investigated scales of magnification, led to the premiss that evolution of structure is continuous (similarity with fractals) and the result of a life-or-death competition for growth between structural units $[29,30]$. As the columnar film increases in thickness, both columns and voids of larger dimensions appear and eventually cluster to form larger similar structures. The voids are regarded as verylow-density regions, rather than completely empty spaces. As a function of the film thickness the so-called growth-death competition yields expanding cones, whereby the slopes of the cones depend on the deposition conditions [29]. Later a more realistic approach was used by adjusting the relation between the film thickness and column size [31]. Whereas in ref. 29 it was assumed to be linear, in ref. 31 simulations were carried out under the assumption of the film thickness being proportional to the square and the cube of the column diameter. Very realistic morphologies were predicted, see Fig. 9. In these simulated structures the initial nucleation sites were distributed randomly and the "growth-or-death" decision when two cones intersected was made at random. An arc of a circle having a radius equal to the film thickness was taken for the top surface curvature of each cone.

Van der Drift [32] made an attempt to consider crystalline aggregation. Selective evolution of the morphology of random and isolated crystals from a plane surface was considered to occur, i.e. each face of the crystallite was shifted parallel to itself by the same amount during film growth and the fastest growing crystallites survived ("survival of the fastest" model). Assumptions made by this model were that a faceted top featured one single crystallite, isotropic supply, and neglect of particle exchange between different faces. The larger the vertical growth rate of a crystallite, the greater was its probability of survival.

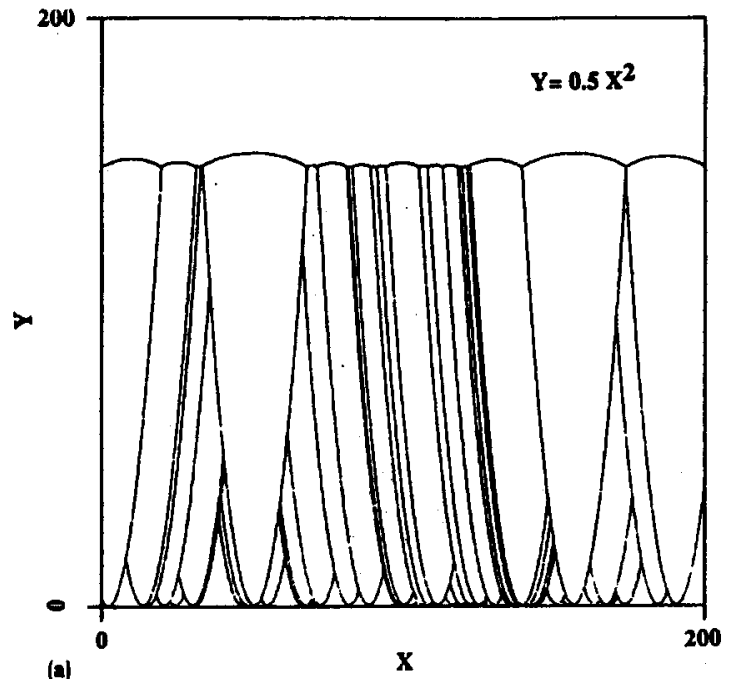

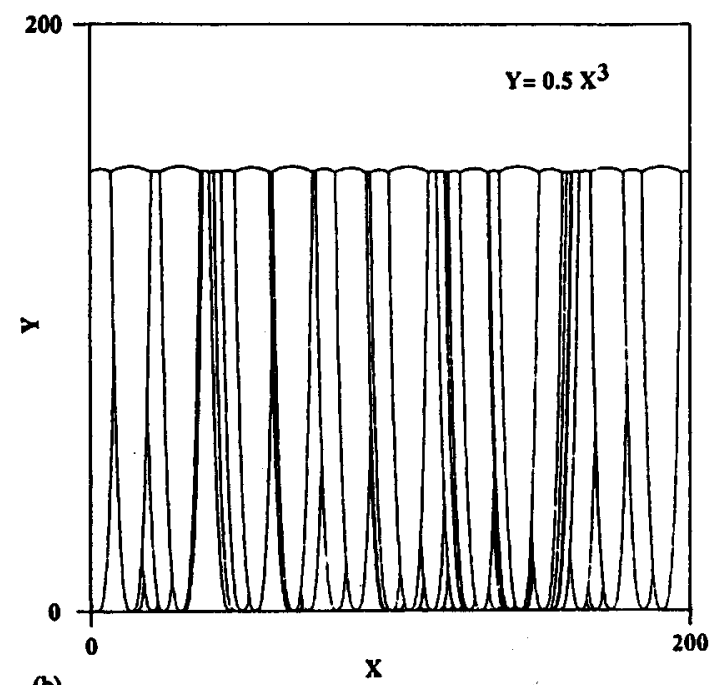

(b)

Fig. 9. Simulated structures for the growth-death competition. The film thickness was proportional to (a) the square and (b) the cube of the column diameter. The units are relative [31]. 
Hence, only a few favoured orientations (with almost maximum vertical growth rate) will survive in the final growth stage and all other orientations would gradually die out. Hergt and Pfeiffer [33] improved this model by allowing diffusional processes between neighbouring faces over corners and crystallite boundary grooves, favouring densely packed net planes. By doing so, the occurrence of multiple textures could be explained, e.g. $\{10 \overline{1} 0\},\{0001\}$ and $\{10 \overline{1} 1\}$ in hcp Co and Co-Cr thin films. (Another extension they made was directed particle supply, to include the influence of the vapour-beam direction. This could explain oblique textures in oblique-incidence deposition, since faces were found favourable that were perpendicular to the beam direction [33].)

Bales and Zangwill [34] formulated a (2-D) thin-film growth model that considered both shadowing effects and surface diffusion, which they called "survival of the fittest". As a result of geometric shadowing, parts of the surface were screened from receiving flux by other parts of the growing surface. Their model was developed for sputtering under high pressure and in the region of $T_{\mathrm{s}} / T_{\mathrm{m}}$ smaller than approximately 0.5 , and it included evaporation under low-mobility conditions. In the absence of surface diffusion, all initial perturbations were found to grow equally regardless of their mutual distance. This coincides with the experiments and model of Messier et al. [29], where repeating and growing morphological features were found with increasing film thickness. By allowing surface diffusion, smoothing occurred, resulting in the column tops becoming flattened. "Survival of the fittest" is derived from the fact that larger columns were preferable to smaller ones (by geometric selection, which was a direct result of the shadowing). With higher surface diffusion, larger columns developed and the vertical roughness decreased. Consequently, the columnar structure developed at a later stage of growth (thicker film) than during lower surface diffusion [34]. With evaporation the impinging particles have lower energy than with sputtering and thus the surface diffusion is less. Therefore evaporated films are expected to have a greater surface roughness than sputtered films. This was observed for evaporated multilayer films of $\mathrm{Cr} / \mathrm{Ta}$ [35]. Furthermore, for these films a close agreement was found with the "survival of the fittest" model.

\section{Growth under oblique-incidence deposition with one source}

With an oblique-incidence vapour flux the shadowing effect plays a substantial role in the film formation; together with the surface mobility, it determines the microstructure of the thin films. The shadowing effect implies that certain parts of the growing film are shadowed for direct impingement. This effect can be quite large in films deposited at oblique incidence. Therefore, the previously-mentioned growth considerations need modifications. It will be shown that an oblique-incidence direction promotes the growth of a columnar morphology and that columnar boundaries are clearer than in normal-incidence films deposited under comparable conditions. Unoccupied shadowed areas may only be filled if diffusion occurs. The discussed and investigated temperature region is such that bulk diffusion can be neglected (the ratio of substrate temperature to melting temperature is below 0.5 ). The surface mobility may have a randomly oriented direction owing to, for example, an elevated film surface temperature, and a pronounced direction due to the oblique-incidence deposition. With two (oblique) vapour directions a two-fold shadowing occurs. Besides this, with nonzero mobility the alloying behaviour of the different materials that are used will also have an influence on the growth. These and other effects will be discussed in this section for 
deposition with a single source. The situation with two sources will be described in Section 5.

\subsection{Shadowing mechanism}

The angle of incidence $\alpha_{\mathrm{i}}$ is defined between the film normal and the arriving vapour flux, see Fig. 1. The simplest case to describe is the one where there is no adatom mobility and where the sticking probability equals one. This means effectively that an incoming atom is captured as soon as it touches a substrate or surface atom. Once it is captured it will then stay at the place where it initially impinged. These conditions are assumed to be valid from here on. Figure 10 illustrates the shadowing effect $[10,36]$ in the two-dimensional case with atoms represented as round hard spheres [37].

In Fig. 10(a) the vapour flux is perpendicular to the substrate $\left(\alpha_{\mathrm{i}}=0^{\circ}\right)$. If a single adsorbed atom is fully exposed to the beam, its capture length $l=4 r$ (with $r$ the radius of the atoms). This means that any atom that passes within a disk diameter of the origin is captured. The parallel vapour-beam trajectories are randomly positioned. Therefore equally probable places for the next captured atom are symmetrically distributed around the origin. With the pair-orientation angle $\psi$ defined as the angular deviation of the original atom plus one captured from the vapour-beam direction (see Fig. 11), it can be easily seen that the mean pair-orientation angle for normal-incidence deposition is $0^{\circ}$. This results in an average chain and columnar inclination angle of $0^{\circ}$. Once there is a situation with the (actual) pair-orientation angle $\psi \neq 0$ (see Fig. 12), the capture length increases $(l>4 r)$. This

a) $\quad \alpha_{1}=0^{\circ}$

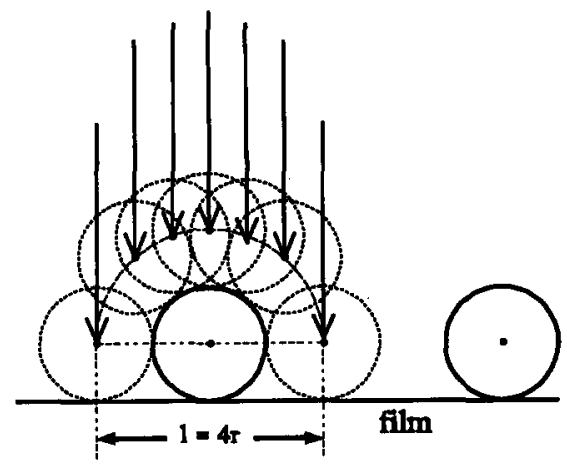

b)

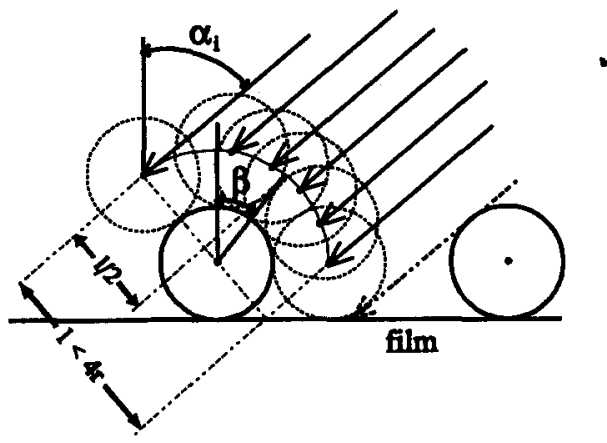

Fig. 10. Illustration of the shadowing mechanism in (a) normal and (b) oblique-incidence deposition. The arrows indicate possible impingement trajectories. The dotted circles are equally probable places for the next captured atom. In (a) the capture length is $4 r$ and the mean pair-orientation angle is perpendicular. In (b) the capture length is shortened by self-shadowing, and consequently the mean pair-orientation angle is shifted away from the incidence angle towards the film normal. (After ref. 37.)

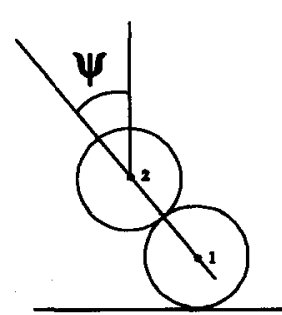

Fig. 11. Definition of pair orientation angle $\psi$. the angular deviation of two captured atoms from the vapour-beam direction. 

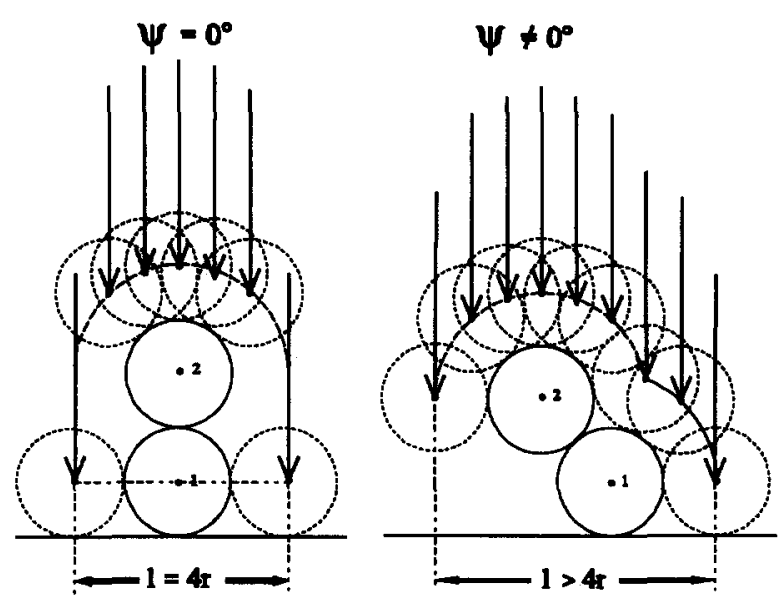

Fig. 12. Capture of the third atom in the normal-incidence deposition. In the left-hand figure the pair-orientation angle is $0^{\circ}$, which makes the capture length $l=4 r$. In the right-hand figure the pair-orientation angle is not equal to $0^{\circ}$, which results in an increase in the capture length: $l>4 r$. This eventually results in chain broadening.

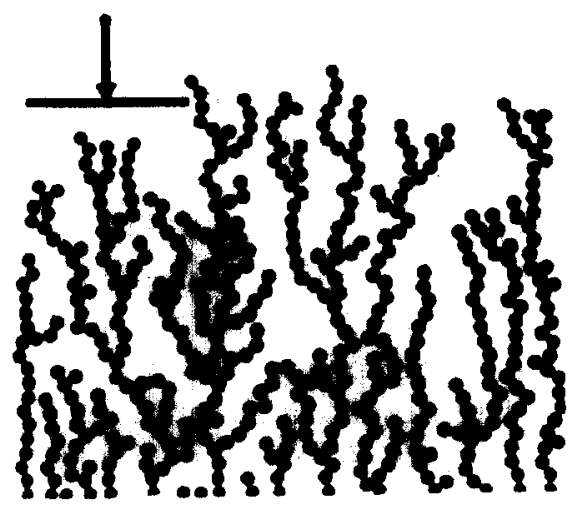

(a)

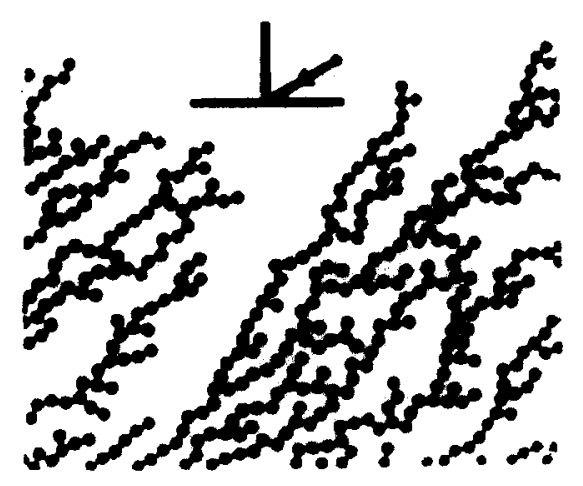

(b)

Fig. 13. 2-D simulation of growth under (a) normal and (b) oblique incidence angles. No relaxation was allowed. In (a) the chain-like structure is nominally perpendicular to the substrate, whereas in (b) it is inclined at an angle between the film normal and the incidence angle [37].

results in broadening of the chain of particles. When the chain gets larger (i.e. longer), frequent splitting and merging of adjacent chains occurs. Figure 13(a) shows a simulation of the structure for normal-incidence deposition under the previously mentioned conditions [37]. Splitting and merging of the chains can be clearly seen.

In the case of a uniform oblique-incidence vapour flux, as can be seen in Fig. 10(b), the capture length is smaller than $4 r$ and is not symmetrical with respect to the two sides of the shadowing atom. Atoms already deposited and surface irregularities throw a shadow, and in these shadowed areas no direct impingement is possible. If we consider the distribution of possible places where a second atom can be captured (dotted circles in Fig. 10(b)), it can be seen that the mean pair-orientation angle is somewhat smaller than the incidence angle. If the number of deposited atoms increases, chains are finally formed, with the chain axis making an angle $\beta$ (less than $\alpha_{\mathrm{i}}$ ) with the film normal. In Fig. 13(b) the outcome of a simulation with oblique-incidence flux $\left(\alpha_{i}=60^{\circ}\right)$ is given. As in Fig. 13(a), chain-splitting 
and merging occur here too. With increasing $\alpha_{\mathrm{i}}$, chain-splitting diminishes and chain branching decreases.

\subsection{Adatom mobility and shape of the nuclei}

With non-zero adatom mobility, it becomes possible for adatoms to move to energetically favourable positions, including the shadowed areas. Therefore an increasing mobility will (partly) annul the shadowing effect. The degree of shadowing and mobility is determined by the deposition conditions (e.g. temperature and contamination of the surface) and the deposited material. Both for normal and oblique-incidence deposition the activation energy for surface diffusion depends on the melting temperature of the material [38]. If the melting temperature is lower, so is the activation energy, and consequently the surface diffusion can be higher.

The direction of adatom movement is considered to have two contributors. The first is (surface) diffusion (e.g. due to a high substrate temperature), which results in a movement in all directions (random) on the surface. The second is the oblique-incidence direction of the vapour atoms $[39,40]$. This causes the atoms to have a momentum component parallel to the incident plane. The atoms will be trapped in a region where their (thermal) energy has become low, e.g. at a kink position, at the edge of a nucleus or due to oxide formation.

Figure 14 illustrates the case of oxide formation for non-noble metals [39]. The $\mathrm{O}_{2}$ supply is assumed to be isotropic over the substrate. Near point $\mathbf{P}$ the metal concentration is at its maximum, and thus the ratio of the oxygen and metal concentration is at its minimum. The distribution of this ratio around the nucleus is given by the dashed line in Fig. 14. As driving forces for the displacement of adatoms, both a gradient of mobility and loss of energy along the contour can be mentioned. In Fig. 14 the critical concentration above which trapping occurs is denoted by the dotted lines $\mathrm{OA}^{\prime}$ and $\mathrm{OB}^{\prime}$. The intersection of the dashed and dotted lines gives the so-called critical trapping points. Beyond these points $\left(A^{\prime}\right.$ and $\left.B^{\prime}\right)$ trapping of the adatoms occurs. Figure 14 represents a situation at the intermediate inclination angle. The location of the critical points drawn here leads to elongated nuclei with the long axis perpendicular to the incidence plane. In cases where the two points $A^{\prime}$ and $B^{\prime}$ are closer to $P$, it is possible to get elongated nuclei with their long axes parallel to the incidence plane. Very low and very high $\alpha_{i}$, lower substrate temperature, higher affinity for oxygen, a higher melting point and a higher partial oxygen pressure are all factors that cause the critical trapping points to move nearer to the point P.

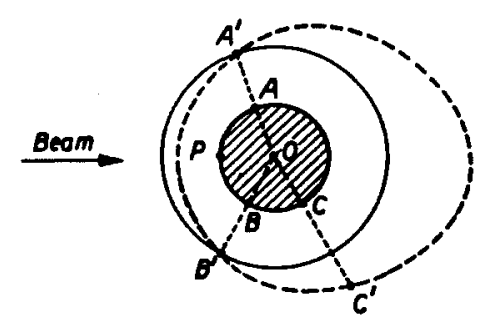

Fig. 14. Adatom trapping due to oxide formation for non-noble metals. A view on top of a nucleus (hatched) is depicted. At point $\mathbf{P}$ there is a maximum in metal concentration. The oxygen concentration is assumed to be isotropic and is represented by the solid line. The dashed line represents the ratio of the oxygen to the metal concentration: e.g. the length of $O C^{\prime}$ represents the oxygen/metal ratio for atoms arriving at $C$. The critical concentration ratio above which trapping occurs is beyond the critical points $A^{\prime}$ and $B^{\prime}[39]$. 
In the literature, reports are made on elongated nuclei both with their long axes perpendicular ( $\mathrm{Sn}, \mathrm{Pt}, \mathrm{Pd}, \mathrm{Ni}-\mathrm{Fe}$ and $\mathrm{Cu}$ [39]) and parallel ( $\mathrm{Ni}-\mathrm{Fe}, \mathrm{Cu}$ and $\mathrm{Sb}$ [39-41]) to the incidence plane. With little adatom mobility and very large directed adatom mobility, an elongated shape with its long axis parallel to the incidence plane is likely to develop. On the other hand, with intermediate mobility the long axis is likely to develop perpendicular to the incidence plane. If the surface diffusion in the random direction increases, then the nuclei will have a greater chance of being symmetrical. As in normally deposited films, with increasing substrate temperature (surface diffusion in the random direction) the density of the nuclei decreases and the crystallite size increases [10].

If the $\mathrm{O}_{2}$ concentration exceeds the $\mathrm{O}_{2} / \mathrm{M}$ concentration (with $\mathrm{M}=$ metal), then a metal atom is trapped by the oxygen atoms. With the $\mathrm{O}_{2}$ concentration assumed to be isotropic over the substrate, the position of the critical trapping points depends on the metal distribution over the substrate/film surface. With an obliquely arriving flux of metal atoms, the metal concentration is at its maximum near point $P$ (see Fig. 14). The position of the critical trapping points thus depends on the displacement of the metal adatoms: with little displacement ("little adatom mobility") and very large displacement ("very large adatom mobility") the metal atoms will be trapped near and very far, respectively, from the point of "supply", i.e. point P. With such conditions, the clustering of metal atoms to the nucleus will result in an elongated shape with its long axis parallel to the incidence plane.

From the above we conclude the following.

As criteria for elongated shape and position of the critical trapping points, we have mentioned deposition parameters that influence the displacement (mobility) and trapping of metal adatoms. The relation between the deposition parameters and adatom mobility is further explained in Section 4.2.1.

Based on the random walk theory (see for example, ref. 42), the diffusion length $L_{\mathrm{s}}$ is given by $L_{\mathrm{s}}{ }^{2}=D_{\mathrm{s}} \times t$, with $t$ the time to deposit one monolayer, $D_{\mathrm{s}}$ the diffusion parameter and $L_{\mathrm{s}}$ the diffusion length, which is the distance that an atom can diffuse before being trapped under successive layers.

\subsubsection{Dependency of the mobility on deposition parameters}

One expects the adatom mobility to be larger for [43]

- smoother crystalline and chemically inert substrates,

- increased substrate temperature during deposition (also refs. 38,44),

- lower deposition rate (also ref. 44),

- higher angle of incidence, and

- lower partial pressure of the ambient gases during deposition.

For example, with lower partial pressures of the ambient gases the mean free path increases and thus the collision chance decreases, thereby imparting more energy to the impinging particles. Also the incorporation of rest-gas particles in the growing film is less likely, as is the probability of oxide formation. (For instance the "impinging rate" of oxygen on the film surface in evaporation with a background pressure of $1.3 \times 10^{-6}\left(1.3 \times 10^{-7}\right)$ mbar is $0.44(0.044)$ monolayer $\mathrm{O}_{2} \mathrm{~s}^{-1}$ ) Both effects mentioned lead to larger adatom mobility. However, it should be noted that some ambiguity may exist. For example, with a higher substrate temperature, on the one hand more energy for surface diffusion is available and adatoms can migrate on the deposit surface before being covered by the arrival of further material. On the other hand, oxidation effects are more likely to occur 
at higher substrate temperatures. This means that for the adatom mobility both increasing and decreasing factors are present. This is also the case with the deposition rate.

\subsection{Distribution of the film thickness}

According to ref. 45 the film thickness of the deposit is given by (surface source; see also Fig. 15):

$h=\frac{m t}{\pi \rho} \frac{\cos \phi \cos \theta}{r^{2}}$

where $m$ is the emitting vapour rate from the source (in $\mathrm{g} \mathrm{s}^{-1}$ ), $\rho$ the material density (in $\left.\mathrm{g} \mathrm{cm}^{-3}\right), \phi$ the direction of the vapour flux, $\theta$ the angle between vapour flux and normal to the receiving surface and $t$ a time unit. Consequently, with varying incidence angles the film thickness varies. In the in-plane direction parallel to the incidence plane this leads to significant thickness variations in obliquely deposited films. For one source in the geometry of Fig. 16, the film thickness variance can be found from:

$h=\frac{m t}{\pi \rho} \frac{\cos ^{2}\left(\alpha_{\mathrm{i}}(b)\right)}{(r(b))^{2}}$

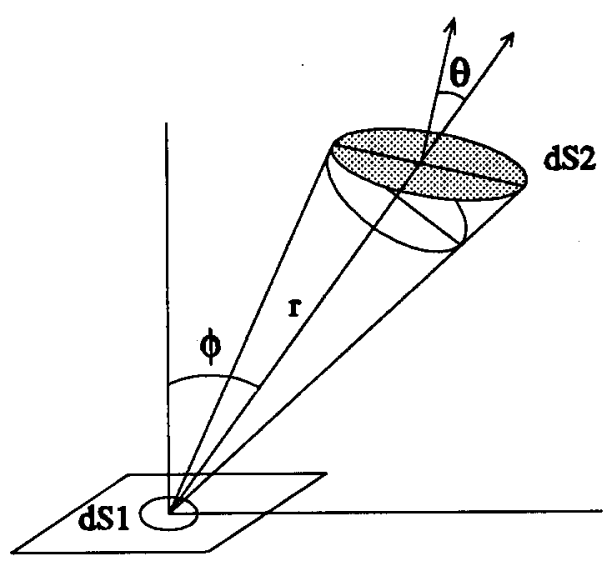

Fig. 15. Evaporation: the normal of the receiving surface dS2 makes an angle $\theta$ with the direction of the vapour beam.

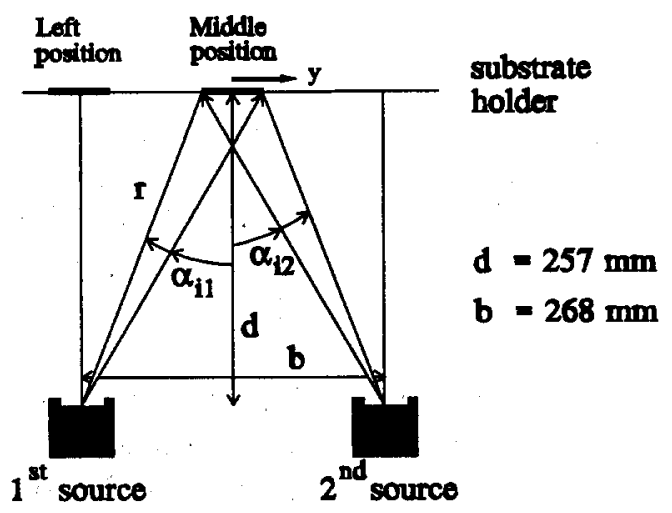

Fig. 16. Basic geometry of the evaporation process [1]. 
$\alpha_{\mathrm{i}}(b)=\tan ^{-1}\left(\frac{0.5 b}{d}\right)$

$r(b)=\sqrt{(0.5 b)^{2}+d^{2}}$

In these expressions $h$ is the film thickness, $\alpha_{\mathrm{i}}$ the angle of incidence and $b, d$ and $r$ geometry parameters (see Fig. 16). The thickness distribution for one source is drawn in Fig. 17 as a function of $y$, the distance measured from the centre of the middle position. The figure displays both calculated and experimental values for Co films. The film thickness for co-evaporated films with two sources can be found by adding up the separate distributions of each source. For such films the film thickness is in agreement with a weighted value of two cosine distributions of two vapour-flux directions. Perpendicular to the $y$ direction the thickness variance can be neglected (here only the distance $r$ changes).

\subsection{Simulation programs for the microstructure}

In order to investigate the structures of thin films and their dependence on the deposition process, various computer simulations have been published in the literature. The simulations of the growth of evaporated films are related to the structure zone models, zones $1, T$ and 2. In terms of the fractal similarities in the structure zone model of Messier et al. [29,30], scaling the dimensions in the simulations to the dimensions in real experiments is no problem [46].

The equilibrium alloy structure of a thin film can be computed by using Monte Carlo (MC) simulation techniques. Monte Carlo simulations are a method for computing averages over a statistical ensemble. They result in the computation of the thermodynamic equilibrium. Contrary to this, molecular dynamics (MD) simulations follow the physical dynamics of the system. However, a problem with MD simulations is that physical processes take a long time with respect to the possible MD computation time. For example, changes in the compositional arrangement of the atoms are extremely slow on the scale of $\mathrm{MD}$ simulations. A clear advantage of MD is that information about the kinetics of the process is obtained, and local compositions can be simulated. Such information is lacking in an MC simulation, since it is only concerned with the equilibrium configurations. When comparing MC simulation results with experiments it is crucial to realize this. Therefore MC simulations of thin-film

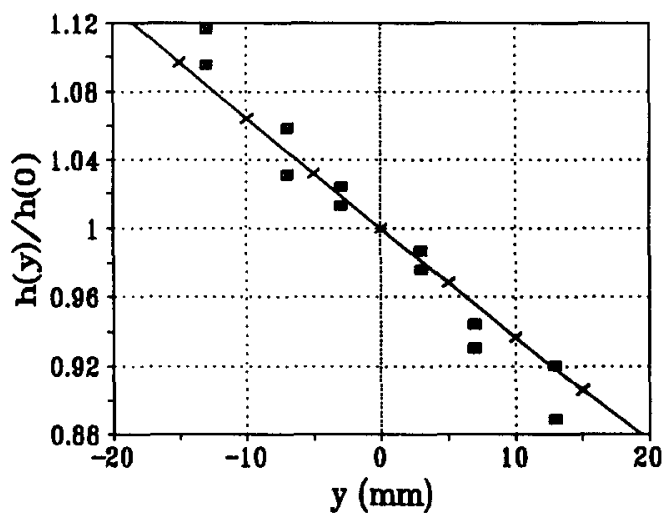

Fig. 17. Distribution of the film thickness due to one source, with $a_{i}=27.5^{\circ}$. The crosses are calculated; the squares are experimental values of a Co thin film. 
growth are only interesting in the low-diffusion region (in terms of the structure zone models, e.g. Movchan-Demchishin model: $T_{\mathrm{s}} / T_{\mathrm{m}}<0.5$ ). A clear advantage of $\mathrm{MC}$ is the possibility of calculating large ensembles on relatively slow and low-capacity computers.

In the case of zone 1 , the particles have limited mobility (or none at all). Shadowing is the governing force of the formation of the film, and ballistic simulations are carried out (the trajectories of incoming particles are nearer to being ballistic than a random course). The particles are considered to be spheres or round disks in the case of threedimensional or two-dimensional simulations, respectively. A particle that is impinging on another one sticks to it or only relaxes to a minor extent, for example into the nearest cradle.

In order to diminish the effects of the edges of the substrate, without needing an enormous amount of calculation time, periodic boundary conditions are presumed. Otherwise only a small part that is away from the edges is suitable for examination.

\subsubsection{Relaxation}

If a captured atom is allowed to move instead of being fixed to the impingement spot, then chains turn into columns. Figure 18 gives a (Monte Carlo simulation) example [37] for relaxation into the nearest cradle (stable position formed by two atoms in the 2-D case). Such a relaxation scheme was first introduced by Henderson et al. [47]. Müller implemented the relaxation scheme by using molecular dynamics simulations, see for instance ref. 48. In this way he could include the impact of ion particles as, for example, occurs in sputtering processes.

\subsubsection{Capture radius}

In an evaporation process, the particles move in straight parallel trajectories towards the film surface (straight because the mean free path is large in comparison to typical dimensions of thin-film structures) until they collide with an already deposited atom or a substrate atom. However, immediately before reaching the film surface, the trajectory of the particle in real evaporation does not remain straight because of the finite interatomic potential (e.g. Van der Waals forces) between the approaching atom and the surface atoms. The deviation from the straight trajectory, which will be a few Ångströms, depends on the particle's kinetic energy and the interaction energy between the surface atoms and the incoming particle. In order to include, to a certain extent, the influence of this effect in

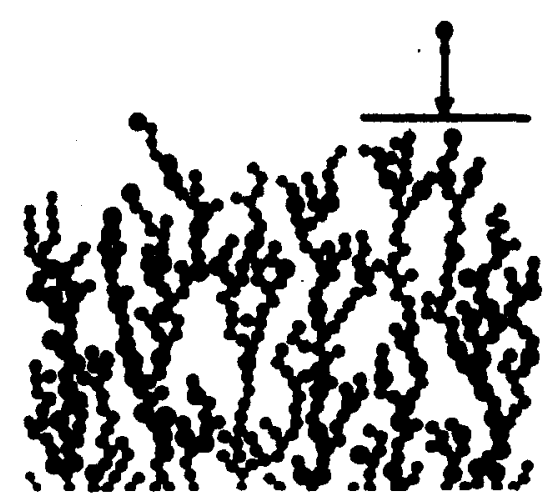

(a)

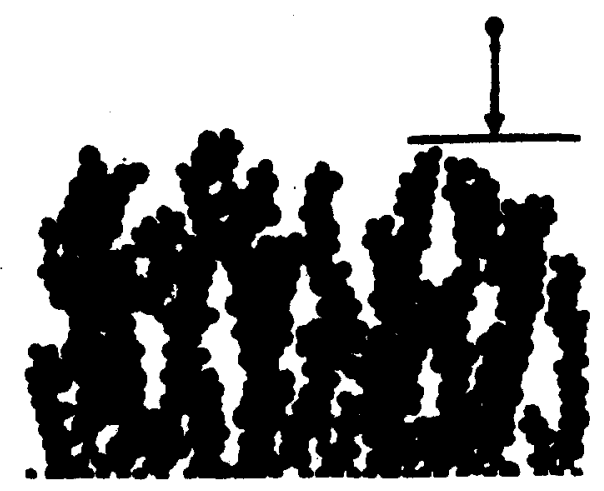

(b)

Fig. 18. 2-D simulation of the growth of a hard-disk alloy $A_{0.2} B_{0.8}, r_{B}=0.8 r_{A}$ under normal incidence. In (a) no relaxation was allowed, whereas in (b) relaxations were included. This resulted in a change from a chain-like structure (a) into a columnar structure (b). The chains and columns are nominally perpendicular to the substrate [37]. 
a simulation model, and taking into consideration the kinetic energy of the incoming particles on the growing structure, the hard-sphere sticking must be replaced [6]. Therefore, we used a hard-sphere sticking model with a capture radius $\left(r_{\mathrm{c}}\right)$ larger than the particle radius $\left(r_{\mathrm{p}}\right)$. In ref. 6 it is derived that a reasonable value for $r_{\mathrm{c}} / r_{\mathrm{p}}$ is between 1.17 and 2.68 . Figure 19 gives an example of simulations with $r_{\mathrm{c}} / r_{\mathrm{p}}=1.25$ and $r_{\mathrm{c}} / r_{\mathrm{p}}=2.0$. Clearly the structure is denser at lower ratios of $r_{\mathrm{c}}$ to $r_{\mathrm{p}}$.

\subsubsection{Substrate}

At the start of a simulation, different (mono-)layers of atoms can be generated which, after the generation is finished, serve as a substrate. For instance, in ref. 49 amorphous and crystalline substrates were used. Also perturbations can be generated, by which hillocks and nodules can be simulated, e.g. ref. 49.

\subsubsection{2-D vs. 3-D and hard-sphere packing}

A 2-D simulation in comparison with the experimental three-dimensional situation yields differences in the average distances between the nuclei, which are too low in the 2$\mathrm{D}$ case, and in the possible directions of diffusion, which are very limited in this case. Secondly, a hard-sphere packing in Monte Carlo does not allow a collective motion of

$$
99
$$

a)

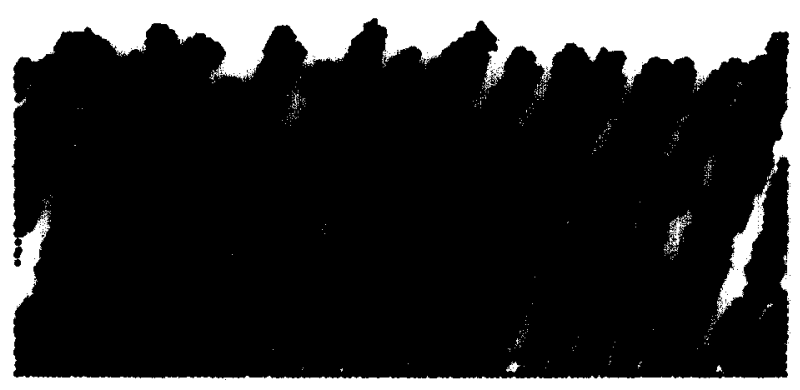

$$
19
$$

b)

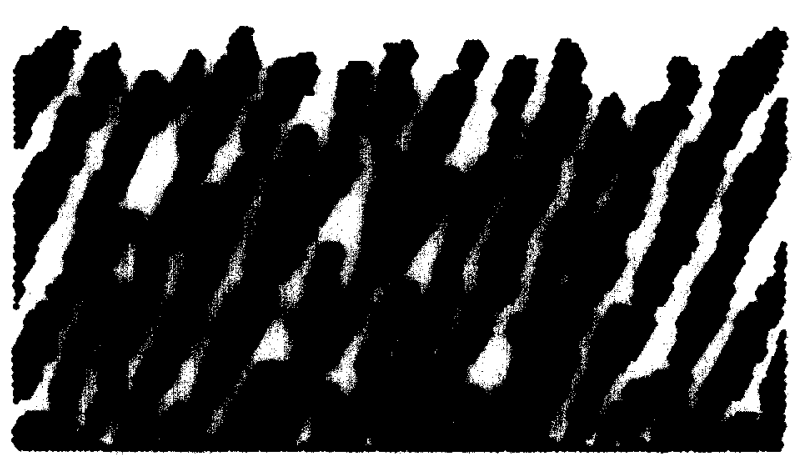

Fig. 19. 2-D Monte Carlo simulation of $\mathrm{Co}_{78} \mathrm{Cr}_{22}$ with relaxation at an incidence angle of $27.5^{\circ}$. In (a) the ratio of the capture radius and particle radius was 1.25 ; in (b) it was 2 [1]. 
atoms. Such a motion would result in the collapse of many open regions, and thus larger columnar sizes could arise. Both effects mentioned lead to smaller dimensions of the simulated 2-D structures as compared to real films. In addition, the limitation of 2-D also prohibits the possibility of simulation of textural features.

\subsubsection{Examples (2-D)}

As examples of 2-D simulations of the growth of Co-Cr films, see Figs. 20 and 21, where $r_{\mathrm{c}} / r_{\mathrm{p}}=1.25$ is taken. Our whole simulated series consisted of the incidence angles $0^{\circ}, 15^{\circ}, 27.5^{\circ}$ and $45^{\circ}$ and variable surface diffusion parameters. The surface diffusion was modelled by thermally induced hopping and is assumed to follow the Boltzmann statistics. Only surface atoms with less than four nearest neighbours were allowed to diffuse, where

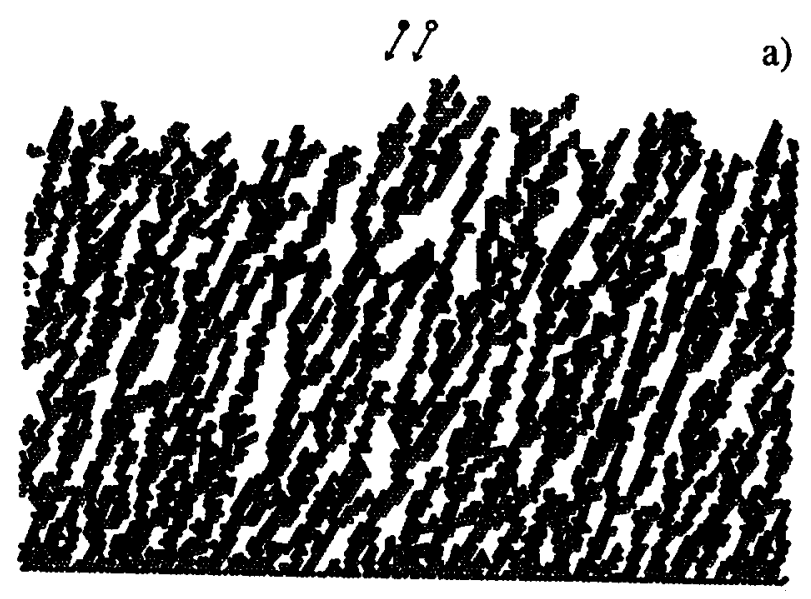

b)

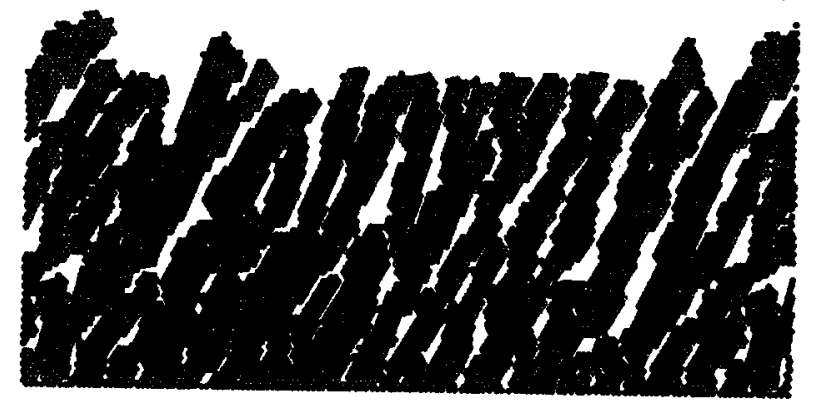

c)

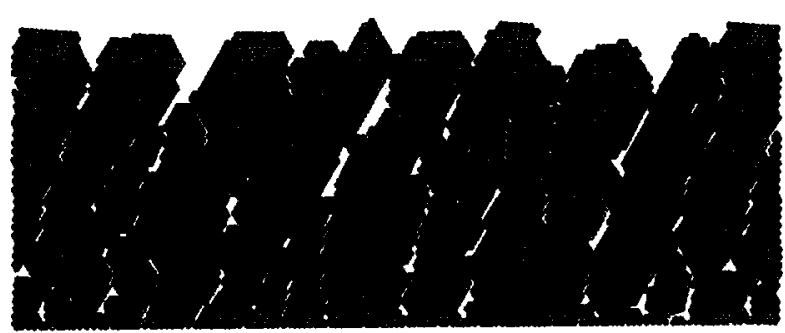

Fig. 20. Simulated $\mathrm{CO}_{78} \mathrm{Cr}_{22}$ structures from a single source with an incidence angle of $27.5^{\circ}$. The solid circles represent the $\mathrm{Cr}$ atoms, the open ones the $\mathrm{Co}$ atoms. From (a) to (c) the degree of surface diffusion increases (the surface diffusion parameter $d_{1}$ for (a), (b) and (c) was 0,73 and 2336, respectively) [1]. 


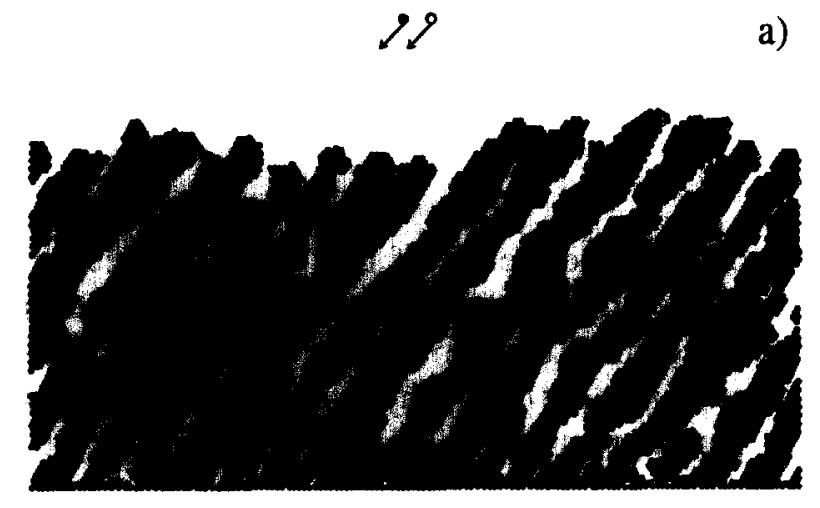

a)

b)

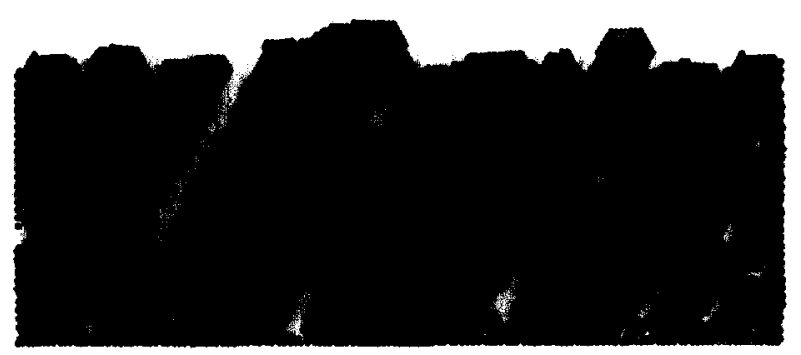

Fig. 21. Simulated $\mathrm{CO}_{78} \mathrm{Cr}_{22}$ structures from a single source with an incidence angle of $45^{\circ}$. The solid circles represent the $\mathrm{Cr}$ atoms, the open ones the Co atoms. In (a) a low diffusion $\left(d_{1}=73\right)$ and in (b) a high diffusion $\left(d_{1}=2336\right)$ are given [6].

nearest neighbours were considered as discs which are at a distance $a$, where $a$ is in the range $2 r_{\mathrm{p}}<\mathrm{a}<\sqrt{ } 5 r_{\mathrm{p}}$. By using the factor $\sqrt{ } 5 r_{\mathrm{p}}$ it is guaranteed that an atom always has less than seven neighbours. The diffusion barrier energy is taken as equal to the sum of the energies of the bonds to be broken for a particular jump. Thus for a particular atom it will depend on the jumping direction, since the sort and number of bonds to be broken can differ for jumps in different directions. The degree of diffusion is indicated by the parameter $d_{1}$, which gives the mean number of diffusional jumps for the most mobile atoms during the time needed for deposition of one monolayer. To give an idea, in a one-component system that is deposited with 1.6 monolayer $\mathrm{s}^{-1}, d_{1}$ values that correspond to $T / T_{\mathrm{m}}$ values (given in brackets) are: $2.5 \times 10^{-5}(0.15), 0.11(0.20), 16(0.25), 460(0.30)$ and $4970(0.35)$.

It was found that the inclination angle of the column-like structures increased with increasing incidence angle for every fixed surface diffusion. Further, as can also be seen in the Figs. 20 and 21, with increasing surface diffusion the inclination angle of the columnlike structures decreased. Both features are in agreement with what can be expected for growth according to the competition between the shadowing mechanism and surface diffusion. The width of the column-like structures increases with increasing surface diffusion, see for instance Fig. 20. The simulated film structures clearly become denser with increasing diffusion, but with an increasing incidence angle the open regions between the column-like structures increase and so the film density decreases. In comparison with evaporated thin films grown under similar mobility conditions, the width of the column-like structures is much too small. Columnar diameters in the real $\mathrm{Co}-\mathrm{Cr}$ films are some $10-50 \mathrm{~nm}$, i.e. some 40-200 atomic diameters. In the simulated structures much smaller dimensions are present. Consequently 
the coherent aggregates of particles are referred to as column-like structures instead of columns. Reasons for this difference of diameter are described above under the sub-section "2-D vs. 3-D and hard sphere packing".

\subsection{Columnar inclination angle}

Nieuwenhuizen and Haanstra [50] measured the dependence of the column direction on the angle of incidence of the evaporation flux. They found that the columnar inclination angle $\beta$ is smaller than the incidence angle $\alpha_{i}$ according to $2 \tan \beta=\tan \alpha_{i}$, the so-called tangent rule (which is based on experimental data only, with no physical background). In experiments it rarely occurs that all deposition parameters match to give compliance with the tangent rule. An example of this is shown in Fig. 22 for obliquely evaporated Fe (single source) [51]. Our own experimental results were not in accordance with the tangent rule, which could most clearly be seen in the dependence of the columnar tilt angle on the substrate temperature (for quantitative values of the columnar tilt angle, the reader is referred to Section 5.2). Our conclusion is that agreement with this rule is an exception. In some simulations of the growth of evaporated films that are governed by self-shadowing, confirmation of the tangent rule was found (e.g. in ref. 52). Other numerical simulations have shown that the tangent rule is only qualitatively, and not quantitatively, correct [53]. In our own simulations no agreement was found.

\subsection{Three-dimensional approach: aggregation of columns and slanting of column tops}

As explained above, an oblique-incidence vapour flux gives rise to the shadowing mechanism. In the incidence plane there is competition between the shadowing mechanism and adatom mobility, which leads to the growth of inclined chains of particles or columns. The columns are separated by open regions, which are incorporated during growth due to the shadowing effect. If the migration rate of the adatoms to the shadowed regions is relatively large, then the open regions will be small; otherwise the resulting film will be porous.

Perpendicular to the incidence plane, i.e. in the transverse plane (for the definition, see Fig. 23), the situation is the same as for normal-incidence deposition. This means no shadowing and a homogeneous supply of vapour. Coalescence is thus likely to proceed perpendicular to the incidence plane. Therefore the columns are likely to be more closely

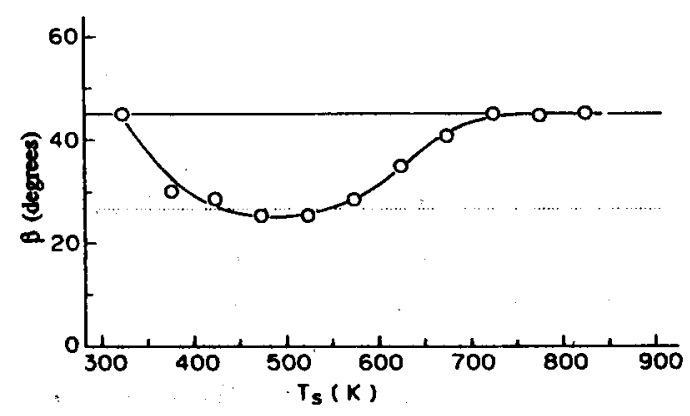

a)

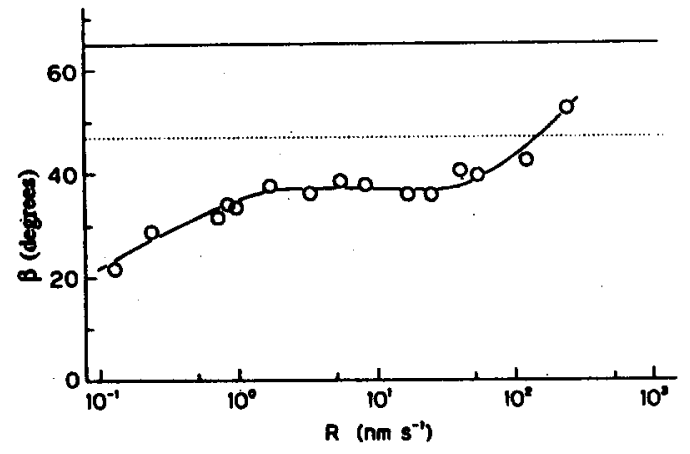

b)

Fig. 22. Columnar-inclination angle for obliquely evaporated $\mathrm{Fe}$ as a function of (a) substrate temperature and (b) deposition rate. The dotted line denotes the columnar inclination angle as depicted by the tangent rule. The solid line denotes the angle of incidence [51]. 


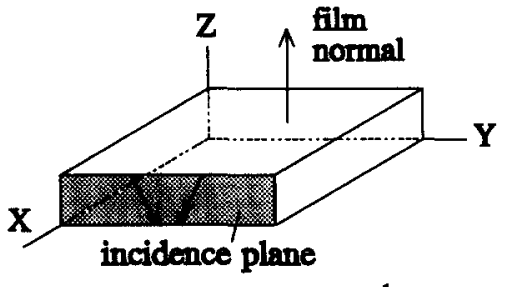

vapour-beam directions: $2^{\text {nd }} \downarrow \downarrow 1^{\text {st }}$

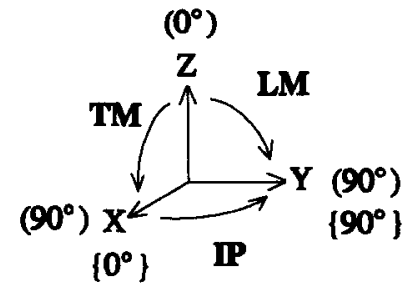

Fig. 23. Definition of the measurement planes: LM longitudinal, TM transverse and IP in-plane measurement. The angles between brackets are valid for TM and LM, those between parentheses for IP. In the case of our co-evaporated films described in Section 5 , the first direction is the direction of the Co vapour $\left(0^{\circ}<\alpha_{i 1}<30^{\circ}\right)$. In the case of our single-source samples, the vapour direction is given by the second direction (and the first direction is absent) [1].

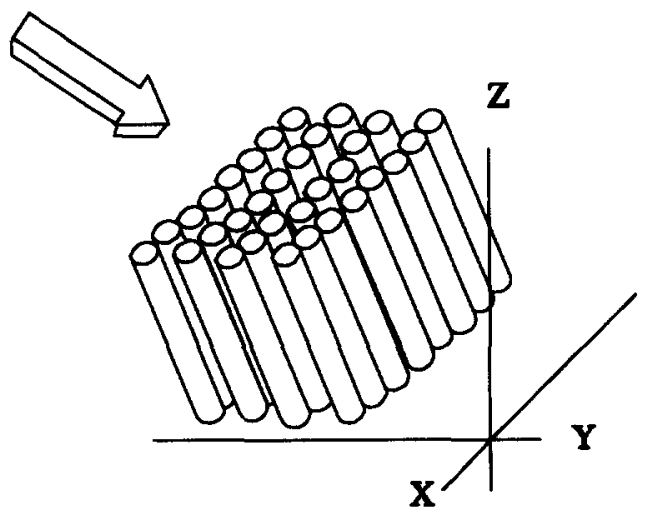

Fig. 24. Anisotropic-column aggregation perpendicular to the incidence plane. In the incidence plane (YZ plane) the shadowing effect causes the columns to be separated by open regions. In the transverse direction ( $X$ direction) there is a homogeneously distributed arrival of vapour atoms. This results in a higher packing density in the $\mathrm{X}$ direction than in the $\mathrm{Y}$ direction. The arrow indicates the vapour-flux direction [1].

packed in the transverse than in the longitudinal direction. This is referred to as anisotropic column aggregation perpendicular to the incidence plane, and it is often called bundling. Figure 24 exemplifies the above. As the angle of incidence increases, the separation between columns in the plane of incidence becomes more pronounced, whereas the separation of the columns measured perpendicular to the plane of incidence is independent of $\alpha_{i}$ [37].

As an experimental example, Fig. 25 shows a replica electron-microscopical observation of obliquely evaporated $\mathrm{Fe}$ from one source [54]. The upper part shows the surface of the film, the lower part the substrate and the middle part shows a cross-section parallel to the incidence plane. The features discussed above, such as column aggregation and "open regions" are displayed in this figure. The morphological differences in the transverse and incident (which is parallel to the arrow in Fig. 25) planes can be clearly seen. A quantitative example of film density is obliquely evaporated $\mathrm{Co}$ (incidence angle, $27.5^{\circ}$ and evaporated on $\mathrm{Si}$ at room temperature). By measuring the geometric film thicknesses (obtained by DEKTAK step profiling measurements) and the volume saturation magnetizations, an indication about the ferromagnetic volume packing is obtained. The ratio of the saturation magnetizations of the obliquely evaporated Co and that of pure hcp Co $\left(=1422 \mathrm{kA} \mathrm{m}^{-1}\right)$ is $0.89-0.94$. Thus a large number of open regions plus possibly oxidized or hydroxidized non-ferromagnetic Co are present in these obliquely evaporated Co films. 


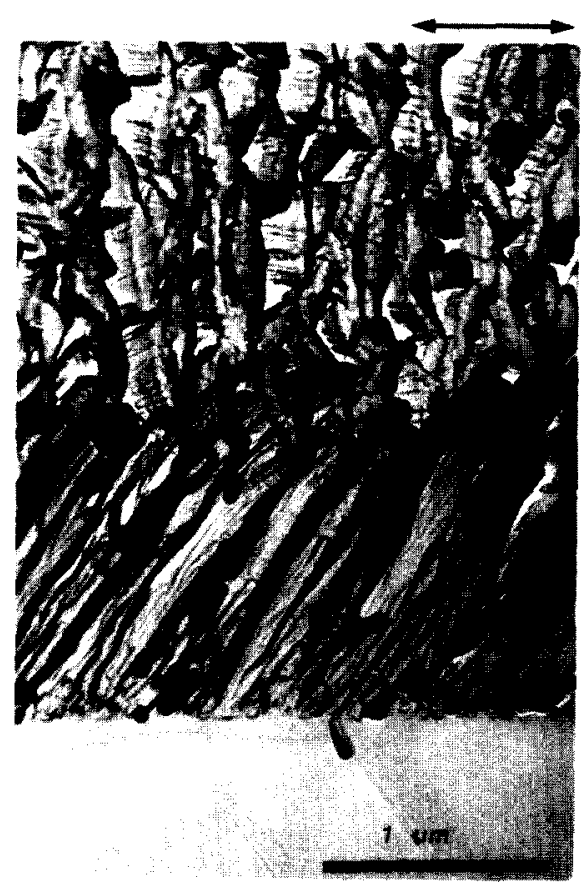

Fig. 25. Replica electron micrograph of an obliquely-evaporated Fe film $\left(\alpha_{\mathrm{i}}=65^{\circ}, T_{\mathrm{s}}=473 \mathrm{~K}\right.$, evaporation rate $=0.83$ $\mathrm{nm} \mathrm{s} \mathrm{s}^{-1}$, background pressure $=0.13 \mathrm{~Pa}$ ). (Taken with permission from ref. 54).

In Section 5 more experimental data will be presented on morphological aspects of obliquely co-evaporated thin films. In the following listing, already one example of a coevaporated film is given.

The appearance of anisotropic column aggregation strongly depends on the deposition conditions. Examples of some experimental results taken from literature are listed below, for pure metal films as well as for alloy films. The metal films involve $\mathrm{Fe}$ and $\mathrm{Co}$ under a wide range of different deposition conditions.

- Obliquely sputtered Fe, $\alpha_{\mathrm{i}}=45^{\circ}$ [55]: $T_{\mathrm{s}}=460-530 \mathrm{~K}$, bundles; $T_{\mathrm{s}}=410-460 \mathrm{~K}$, no bundles.

- Obliquely evaporated $\mathrm{Fe}, \alpha_{\mathrm{i}}=80^{\circ}$ [56]: at a background pressure of $2.7 \times 10^{-3} \mathrm{~Pa}$, no bundles were present, and the highest packing density of the columns was parallel to the incidence plane.

- Obliquely evaporated Fe, $\alpha_{\mathrm{i}}=60^{\circ}, 4 \times 10^{-3} \mathrm{~Pa}$ [57]: $T_{\mathrm{s}}<240 \mathrm{~K}$ and $T_{\mathrm{s}}>320 \mathrm{~K}$, bundles, $240 \mathrm{~K}<T_{\mathrm{s}}<320 \mathrm{~K}$, densely packed structure.

- Obliquely evaporated Fe, $\alpha_{\mathrm{i}}=60^{\circ}, 4 \times 10^{-3} \mathrm{~Pa}, h>0.8 \mu \mathrm{m}$ [58]: $T_{\mathrm{s}}<650 \mathrm{~K}$, bundles; $T_{\mathrm{s}} \geqslant 650$ $K$, dense and isotropic packing density.

- Obliquely evaporated Fe, $R=0.33 \mathrm{~nm} \mathrm{~s}{ }^{-1}, P=6.7 \times 10^{-3}-6.7 \times 10^{-5} \mathrm{~Pa}, h=100 \mathrm{~nm}$ [59]: at $\alpha_{i}=75^{\circ}$ elongated particles parallel to the incidence plane are observed. Based on the in-plane coercivity, a similar elongated structure was expected to be present at lower incidence angles $\left(0^{\circ}-75^{\circ}\right)$.

- Obliquely evaporated $\mathrm{Co}, \alpha_{\mathrm{i}}=80^{\circ}$ [60]: elongated structure parallel to the incidence plane.

- Obliquely evaporated $\mathrm{Co}, \alpha_{\mathrm{i}}=70^{\circ}, T_{\mathrm{s}}=200^{\circ} \mathrm{C}$ [61]: $R=3.83 \mathrm{~nm} \mathrm{~s}^{-1}$, bundles; $R=0.12 \mathrm{~nm}$ $\mathrm{s}^{-1}, R=1.83 \mathrm{~nm} \mathrm{~s}^{-1}$, no bundles.

- Obliquely evaporated $\mathrm{Co}, \alpha_{\mathrm{i}}=60^{\circ}, 4 \times 10^{-3} \mathrm{~Pa}$ [62]: $T_{\mathrm{s}}=213 \mathrm{~K}$ and relatively high $R$ (" $R_{\mathrm{v}}$ " $=91 \mathrm{~Hz} \mathrm{~s}^{-1}$ with $R_{\mathrm{v}}$ the rate of change in the resonant frequency of a quartz crystal 
oscillator and $R_{\mathrm{v}}=270 \mathrm{~Hz} \mathrm{~s}^{-1}$ corresponds to $R=8 \mathrm{~nm} \mathrm{~s}^{-1}$ ), bundles; $T_{\mathrm{s}}=213 \mathrm{~K}$ and relatively low $R\left(R_{\mathrm{v}}=17 \mathrm{~Hz} \mathrm{~s}{ }^{-1}\right)$, no bundles; $T_{\mathrm{s}}=273 \mathrm{~K}$, hardly any bundles present, independent of the deposition rate $R$.

- Obliquely evaporated $\mathrm{Cu}, \alpha_{\mathrm{i}}=0^{\circ}, 30^{\circ}, 60^{\circ}, 85^{\circ}, h=50 \mathrm{~nm}, R=2 \AA \mathrm{s}^{-1}, 4 \times 10^{-3} \mathrm{~Pa}$ [63]: no shape elongation nor column aggregation.

- Obliquely evaporated $\mathrm{Co}_{70} \mathrm{Fe}_{30}, \alpha_{\mathrm{i}}=70^{\circ}-85^{\circ}, T_{\mathrm{s}}<100^{\circ} \mathrm{C}, h=40 \mathrm{~nm}$ [64]: elongated particles parallel and bundling perpendicular to the incidence plane occurred simultaneously.

- Obliquely evaporated $\mathrm{Co}_{1-x} \mathrm{Ni}_{x}, \alpha_{\mathrm{i}}=60^{\circ}, T_{\mathrm{s}}=213 \mathrm{~K}[65]: x<0.6$, bundling occurred. With increasing $x$ the degree of bundling became less and the surface became smoother and more isotropic.

- Obliquely evaporated $\mathrm{Co}-\mathrm{Cr}, \alpha_{\mathrm{i}}=20^{\circ}-70^{\circ}, R=1 \mathrm{~nm} \mathrm{~s} \mathrm{~s}^{-1}$ and $10 \mathrm{~nm} \mathrm{~s} \mathrm{~s}^{-1}$ [66]: bundles occurred, but also an elongated shape of the columns with the long axis of elongated particles parallel to the incidence plane. The bundles were more strongly present at higher incidence angles. A heat treatment changed the anisotropy in the low-rate samples (1 $\mathrm{nm} \mathrm{s}^{-1}$ ) into a stronger anisotropy parallel to the incidence plane, which was attributed to irreversible changes of the microstructure.

- Obliquely evaporated $\mathrm{Ni}-\mathrm{Fe}, \alpha_{\mathrm{i}}=85^{\circ}$ [9]: elongated crystallites parallel to the incidence plane.

- Obliquely evaporated $\mathrm{Cd}-\mathrm{Te},\left(\alpha_{\mathrm{i}}, \mathrm{T}_{\mathrm{s}}\right)=\left(70^{\circ}, 250^{\circ} \mathrm{C}\right),\left(80^{\circ}, 150^{\circ} \mathrm{C}\right),(4-5) \times 10^{-3} \mathrm{~Pa}[67]$ : bundles.

- Obliquely co-evaporated $\mathrm{Fe}_{80} \mathrm{~B}_{20}, \alpha_{\mathrm{i}}=50^{\circ}$ [68]: bundles.

The main point from these results from the literature is that bundles only occur in a limited region of deposition parameters. This is also true for the appearance of elongated particles parallel to the incidence plane. Non-bundling may be most easily attained under conditions of higher adatom mobility, since in this case the density of the nuclei is less and their dimensions and separation are larger. The most favourable conditions for an increased separation between columnar units are thus given by a higher ratio of substrate and melting temperatures, a lower deposition rate, lower partial pressures and higher incidence angles.

Formation of surface roughness is inherent to the shadowing mechanism. The growth of nuclei surfaces proceeds with tilted surfaces (see solid lines in Fig. 26 that represent subsequent surface topography). Once a nucleus reaches the geometric shadow of a second one, the lateral growth of the first will stop through lack of supply. The growth of the first

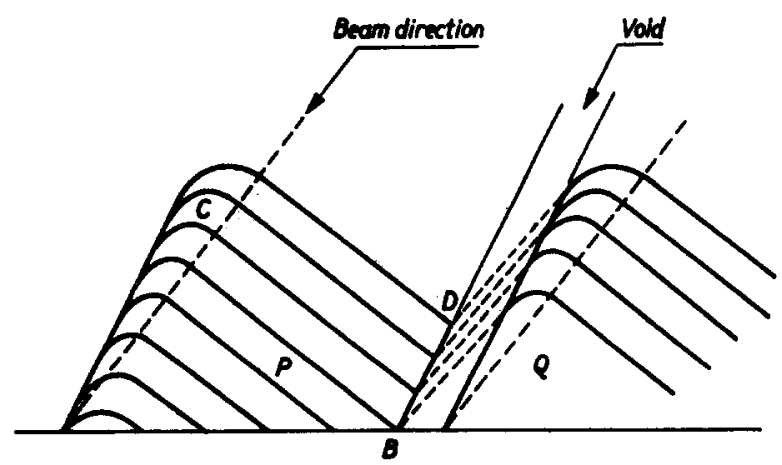

Fig. 26. Illustration of surface roughness and open regions between columns that grow obliquely owing to the shadowing effect. When nucleus $P$ reaches the shadow of nucleus $Q$, which is at front $B$, the lateral growth of nucleus $P$ stops and its surface front grows parallel to itself [39]. 
nucleus then proceeds roughly parallel to the shadow side of the second one [39]. Slanting of the column tops thus occurs towards the vapour beam direction. In Section 5 an experimental observation of this is given for co-evaporated $\mathrm{Co}-\mathrm{Cr}$.

\subsection{Texture}

The texture can influence the morphology, and the object of this sub-section is to give some examples. There are uniaxial and biaxial textures, which are also called fibre and sheet textures. In the case of a fibre texture, only one crystallographic axis (of most crystals) points in a given direction (i.e. $\|$ fibre axis). In the case of a sheet texture, two axes of most crystals point in preferred directions. The notation is then two-fold; the square brackets and parentheses indicate the crystallographic direction parallel to the texture axis and the crystallographic plane parallel to the incidence plane, respectively [69]. Owing to the texture properties, different structures can grow. Fe evaporated obliquely at $65^{\circ}$ at $473 \mathrm{~K}$ by Fujiwara's group [70] is taken as an example. At intermediate deposition rates $\left(1.5<R<30 \mathrm{~nm} \mathrm{~s}^{-1}\right)$ a type $I$ [111](110) texture is present. In this case (most of) the top surfaces of the columns turn away from the vapour source. The $\{100\}$ planes cause the newly arrived adatoms to move downwards, and the structure that grows has a higher packing density of the columns parallel to the incidence plane. However, Fe evaporated at low and high deposition rates has type II [111](110) and [100](010) textures. This coincides with habit planes on the top of a column in which bundles are formed that face towards the vapour source. The adatom movement could thus be in planar directions. The highest packing density, in this case, was perpendicular to the incidence plane and bundling was observed.

A second example is obliquely deposited $\mathrm{Co}$, also from the Fujiwara group [71,72]. In sputtered Co $\left(\alpha_{\mathrm{i}}=45^{\circ}, h=0.8 \mu \mathrm{m}, R=0.4 \mathrm{~nm} \mathrm{~s}^{-1}\right)$ at an argon pressure of $20 \mathrm{~Pa}$ and a substrate temperature below $360 \mathrm{~K}$, a coexistence of an hcp and an fcc structure was present. The fcc structure was [110](111): the [110] axis inclined from the film normal towards the beam direction by $10^{\circ}$ and the (1ii) plane was parallel to the incidence plane. The $c$ axis for the hcp structure was found to lie in-plane in the direction perpendicular to the incidence plane (transverse direction). As for the corresponding morphology, crystallites were elongated parallel to the incidence plane. There was an hcp structure in the evaporated Co $\left(\alpha_{\mathrm{i}}=60^{\circ}\right.$, $h=0.5 \mu \mathrm{m}, R=8 \mathrm{~nm} \mathrm{~s}^{-1}$, background pressure $4 \times 10^{-3} \mathrm{~Pa}$ ) with substrate temperatures below $280 \mathrm{~K}$. The morphology showed bundles, being clustered crystallites perpendicular to the incidence plane.

A controversial subject is the dependence of the condensation coefficient on the angle of incidence. The condensation coefficient represents the chance of an atom becoming adsorbed or condensed on the crystal surface. When the angle between the vapour beam and the normal to the crystal surface is small, the proposed coefficient is also small. According to Bauer [69] this causes preferential growth of certain crystal planes, but according to the Fujiwara group this is not the case for $\mathrm{Fe}$ [58]. In their investigation it appeared that the adatom movement taking place after the condensation determined the growth and texture evolution.\}

\subsection{Switch of the effective in-plane anisotropy direction}

The anisotropic morphological dimensions in-plane yield an in-plane shape anisotropy. In Section 4.6 it was stated that aggregates of columns can form in the transverse (inplane) direction, the so-called bundles. In the longitudinal direction the columns are separated by open regions. Hence, an in-plane shape anisotropy develops in the transverse direction which is referred to as $K_{\mathrm{t}}$. 
With increasing incidence angle $\alpha_{\mathrm{i}}$, the columnar morphology develops more pronouncedly. Open regions between columns get wider. Consequently the columnar-shape anisotropy, which is directed along the columnar axis, becomes stronger. This anisotropy contributes to the total out-of-plane anisotropy, possibly together with a textural anisotropy along, for instance, a fibre axis. Therefore with the columnar-shape anisotropy getting stronger, the out-of-plane anisotropy increases. Because in most obliquely deposited films the out-ofplane anisotropy is tilted with respect to the film normal, there is an in-plane anisotropy energy component which is parallel to the incidence plane. Hence, with increasing incidence angle, the component of the in-plane anisotropy energy becomes larger.

At a certain critical incidence angle, it is possible that the in-plane anisotropy energy component of the tilted out-of-plane anisotropy becomes stronger than the transverse inplane anisotropy $K_{\mathrm{t}}$. When this happens, the effective in-plane anisotropy direction changes from the transverse direction for $\alpha_{\mathrm{i}}<\alpha_{\mathrm{i} \text { CRITICAL }}$ into the longitudinal direction for $\alpha_{\mathrm{i}}>\alpha_{\mathrm{i} \text { CRITICAL }}$. This switch is, for instance, visualized for Ni-Fe in Fig. 2 by the zero crossing. It is also measured for other metals, for instance Co [73] and Fe [74], and it is even calculated by Alameda et al. [75], who made use of a phenomenological packing parameter for the bundles.

\subsection{Oblique evaporation with continuously varying incidence angle: $M E$ tape}

An interesting applied deposition technique in industry for preparing thin-film media on flexible substrates is the use of a so-called roll coater, see Fig. 27. The substrate is continuously supplied with coating material and is transported by a rotating drum [76]. The deposition of the thin film takes place by evaporation of the source material. The evaporation is not done at a fixed angle, since the incidence angle changes as the substrate passes along the vapour flux. Therefore this deposition process is often called CVI: continuously varying incidence. Evaporation is chosen in preference to sputtering because of the much higher deposition rates that can be achieved in evaporation. An evaporation rate of $1 \mu \mathrm{m}$ $\mathrm{s}^{-1}$ is realized [77], and a high throughput is therefore possible. The vapour flux is oblique

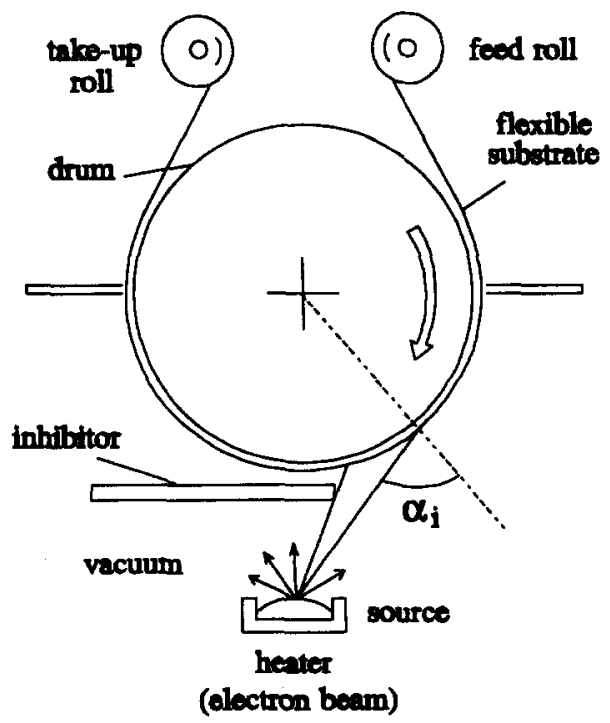

Fig. 27. Industrial application of oblique-incidence evaporation (ME tape mass-production equipment). 
and the shield limits the incidence angles of vapour arriving on the growing film surface. The incidence-angle region must be chosen largely from the point of view of low material loss. However, the desired film properties can only tolerate small deviations, which puts a limit on the incidence-angle variation.

In 1989 metal evaporated tape (ME tape) appeared on the market, being a thin-film medium on a flexible substrate for application in the analogue high-band $8 \mathrm{~mm}$ (Hi8) video system. This medium is $\mathrm{Co}-\mathrm{Ni}$ evaporated obliquely in an oxygen environment using a roll coater. The average composition is $\left(\mathrm{Co}_{80} \mathrm{Ni}_{20}\right)_{80} \mathrm{O}_{20}$, and typical film thicknesses are $200 \mathrm{~nm}$ with a $4 \mathrm{~nm}$ rust protection layer on the surface. The production process of ME tape is modified with respect to oblique evaporation with a stationary substrate in order to obtain an efficient use of the evaporated material. In addition it allows for continuous evaporation of hundreds of metres of tape in a single run at a very high production speed. The Co$\mathrm{Ni}-\mathrm{O}$ particles grow in inclined columns, varying their tilt angle with the changing incidence angle. The presence of non-ferromagnetic Co oxides assures the lowering of the exchange coupling between the ferromagnetic (Co-Ni) particles, which is necessary for obtaining a particulate behaviour. The easy direction of magnetization is inclined out of the film plane $\left(20^{\circ}\right.$ if the shape anisotropy of the film plane is included, and about $35^{\circ}$ if it is excluded [78]).

\subsection{Summary}

It can be concluded from Section 4 that the growth of the obliquely-deposited films of interest $\left(T_{\mathrm{s}}<0.5 T_{\mathrm{m}}\right)$ is predominantly determined by the competition of the shadowing mechanism and surface diffusion. The surface diffusion consists of adatom mobility and can have a directed component parallel to the in-plane projection of the obliquely incoming vapour flux direction. It can further have a random direction and depends on the melting temperature $T_{\mathrm{m}}$ of the material [38]. With lower $T_{\mathrm{m}}$ the activation energy for surface diffusion is less and consequently the surface diffusion can be higher.

Being able to control the shadowing mechanism and surface diffusion means that the growth of the thin film can be more or less tailored. It is therefore important to realize how these factors can be manipulated. The shadowing is caused by obliquely (i.e. nonperpendicular to the receiving surface) arriving particles. Hence the angle of incidence, its distribution and surface irregularities are the tools by which the shadowing is triggered. The degree of surface diffusion first of all depends greatly on the material because of its melting temperature and its affinity to partial gases (like oxygen). In addition, all deposition parameters influence the mobility. The incidence angle mainly contributes to the directed momentum in the longitudinal direction. Further there are: the substrate temperature (or better the ratio of $T_{\mathrm{s}}$ and $T_{\mathrm{m}}$ ), the deposition rate, the background pressure, the partial gases and their pressures, and (especially important for the nucleation) the crystallinity, cleanness and reactivity of the substrate.

Finally in Section 4.9, commercially used ME tape for magnetic recording has been described briefly.

\section{Growth under oblique-incidence deposition with two sources}

In the case of two separate vapour-flux directions, instead of one, the resulting morphology is determined by the two incidence angles, the partial deposition rates, the ratio of the radii and the atomic weight of the two materials used [2,3]. A weighted average of these factors yields an effective incoming vapour direction. On a macroscopical scale, all char- 
acteristics mentioned for single-source evaporated films can be transferred to the multiplesource case if the effective incoming vapour-beam direction is taken into account $[2,52,68,79,80]$. Therefore the specific features that can occur in obliquely-deposited films are not repeated in this section, and the reader is referred to Section 4. Naturally, results on these features will be discussed.

If the effective incoming vapour-beam direction is not parallel to the film normal, then the resultant oblique flux promotes the growth of a tilted columnar structure where the columns are inclined towards the effective incoming direction. As for the single-source case (see Section 4) growth proceeds according to the competition between the shadowing mechanism and surface diffusion. Besides a thermally induced random direction, the surface diffusion may include a pronounced direction parallel to the incidence plane due to the oblique incoming direction [81]. Hence, elongated shapes of the columns can occur.

In this section we will focus our analysis and discussion on obliquely co-evaporated thin-film media for magnetic recording. Since we will use (new) experimental data for samples prepared in our laboratory, first our evaporation parameters will be given in Section 5.1. Then in Section 5.2 the morphology of co-obliquely deposited films is presented. Differences for single- and dual-source oblique deposition will be discussed in this section. Section 5.3 deals with another aspect that can be exploited when using two opposing oblique directions, namely (tailoring) the local composition. This is even possible at room temperature, which is the main advantage for application of deposition with two sources instead of one. However, at present, a disadvantage is that the co-deposition takes place at low deposition rates. We investigated the aspect of tailoring the local composition because, in thin films for magnetic recording, local regions with less- or non-ferromagnetic regions are desirable for a good magnetic performance. The anisotropic morphology (Section 5.2) and the anisotropic local composition (Section 5.3) will be shown to exist in our obliquely co-evaporated thin films which were prepared for recording applications. In order to explain this, we will also use some magnetic parameters. Since the anisotropic magnetic effects are, in fact, based on anisotropic microstructural effects, the exploitation of the co-oblique vapour fluxes can also be used to tailor desired anisotropic optical or, for example, electrical parameters. Section 5.4 summarizes our ideas on this.

\subsection{Evaporation parameters}

Our experimental data concern obliquely (co-)evaporated films. The basic geometry of the process is illustrated in Fig. 16. Two E-beam sources are available to heat two separate sources. The two vapour beams come from different directions. At least one of them arrives at the substrate and film surface under a non-zero angle (all angles are defined with respect to the film normal), the so-called oblique deposition. The incidence plane is given by the substrate normal and the vapour direction(s). The angle of incidence $\alpha_{\mathrm{i}}$ is defined between the film normal and the vapour-flux direction. The substrate is located in the incidence plane (for our samples also called the evaporation plane) and can be placed above either one of the sources and at all positions in between, thus enabling symmetric $\left(\alpha_{i 1}=\alpha_{i 2}\right)$ and asymmetric $\left(\alpha_{i 1} \neq \alpha_{i 2}\right)$ depositions. In the former case the incoming vapour directions are opposite. We used two different substrate positions: precisely between the two sources and exactly above the Co source. The first series are the co-evaporated symmetrically deposited ones, with $\alpha_{\mathrm{i} 1} \approx \alpha_{\mathrm{i} 2} \neq 0^{\circ}$. These have a code name starting with $\mathrm{M}$ (Co-Cr on Si substrates), F (Co-Cr on polyimide substrates), A (Co-Ag) or T (Co-Ta): see Table 1 . In order to make a comparison, there are samples deposited from a single source, which have a code name 
TABLE 1. Illustration of the code names of the samples

\begin{tabular}{|c|c|c|c|c|c|c|}
\hline $\begin{array}{l}\text { First letter } \\
\text { of code name }\end{array}$ & $\begin{array}{l}\text { Thin film } \\
\text { material }\end{array}$ & $\begin{array}{l}\text { Specification of } \\
\text { evaporation geometry }\end{array}$ & $\alpha_{\mathrm{iCo}}$ & $\alpha_{\mathrm{ix}}{ }^{*}$ & $\alpha_{i \mathrm{Ca}-\mathrm{Cr}}$ & Substrate \\
\hline $\mathbf{M}$ & $\mathrm{Co}-\mathrm{Cr}$ & Dual source; $\alpha_{i 1} \approx \alpha_{i 2}$ & 27.5 & 27.5 & - & $(100) \mathrm{Si}$ \\
\hline $\mathbf{F}$ & $\mathrm{Co}-\mathrm{Cr}$ & Dual source; $\alpha_{i 1} \approx \alpha_{i 2}$ & 27.5 & 27.5 & - & Polyimide \\
\hline $\mathbf{A}$ & $\mathrm{Co}-\mathrm{Ag}$ & Dual source; $\alpha_{i 1} \approx \alpha_{i 2}$ & 27.5 & 27.5 & - & $(100) \mathrm{Si}$ \\
\hline $\mathbf{T}$ & $\mathrm{Co}-\mathrm{Ta}$ & Dual source; $\alpha_{i 1} \approx \alpha_{i z}$ & 27.5 & 27.5 & - & $(100) \mathrm{Si}$ \\
\hline $\mathbf{S}$ & $\mathrm{Co}-\mathrm{Cr}$ & Single source & - & - & 27.5 & $(100) \mathrm{Si}$ \\
\hline $\mathbf{L}$ & $\mathrm{Co}-\mathrm{Cr}$ & Non-rotating substrate: $\alpha_{i 1}=0^{\circ}$ & 0 & 46 & - & $(100) \mathrm{Si}$ \\
\hline LR & $\mathrm{Co}-\mathrm{Cr}$ & Rotating substrate; $\alpha_{i 1}=0^{\circ}$ & 0 & 46 & - & $(100) \mathrm{Si}$ \\
\hline
\end{tabular}

$\mathrm{X}$ is used for $\mathrm{Cr}, \mathrm{Ag}$ and $\mathrm{Ta}$.

TABLE 2. Quotients of substrate temperature $T_{s}$ and melting temperature $T_{m}$ of four different metals

\begin{tabular}{lllllll}
\hline Element & $\begin{array}{l}T_{\mathrm{m}} \\
(\mathbf{K})\end{array}$ & $T_{\mathrm{s}}=25^{\circ} \mathrm{C}$ & $T_{\mathrm{s}}=50^{\circ} \mathrm{C}$ & $T_{\mathrm{s}}=100^{\circ} \mathrm{C}$ & $T_{\mathrm{s}}=150{ }^{\circ} \mathrm{C}$ & $T_{\mathrm{s}}=200{ }^{\circ} \mathrm{C}$ \\
\hline $\mathrm{Co}$ & 1768 & 0.17 & 0.18 & 0.21 & 0.24 & 0.27 \\
$\mathrm{Cr}$ & 2130 & 0.14 & 0.15 & 0.18 & 0.20 & 0.22 \\
$\mathrm{Ag}$ & 1235 & 0.24 & 0.26 & 0.30 & 0.34 & 0.38 \\
$\mathrm{Ta}$ & 3269 & 0.09 & 0.10 & 0.11 & 0.13 & 0.14 \\
\hline
\end{tabular}

starting with $\mathrm{S}$ (all $\mathrm{Co}-\mathrm{Cr}$ on $\mathrm{Si}$ substrates). Finally there are samples deposited above the Co source, all being $\mathrm{Co}-\mathrm{Cr}$ films on $\mathrm{Si}$ substrates. These are either made on a non-rotating substrate (code names starting with $\mathrm{L}$ ), or a rotating substrate (code names starting with $\mathrm{LR}$, rotation speeds 30 and $82 \mathrm{rev} \mathrm{min}^{-1}$ ). The Si substrates are all (100) $\mathrm{Si}$; the thickness of the polyimide substrates is $50 \mu \mathrm{m}$ or $12.5 \mu \mathrm{m}$.

The total deposition rate was varied between 2 and $13 \AA \mathrm{s}^{-1}$. The separate deposition rates of both sources during deposition were monitored by calibrated quartz crystal oscillators.

The deposition temperature was either ambient (room) temperature (referred to as $T_{\mathrm{R}}$ samples) or high (referred to as $T_{\mathrm{H}}$ samples). In the latter case the temperature near the heater, $T_{\mathrm{p}}$, was 300 or $400{ }^{\circ} \mathrm{C}$. The substrate temperature, measured by means of a thermocouple in contact with the back of the Si substrates, was lower than this heater temperature. After heating for $1 \mathrm{~h}$, which was the minimum heating time used, it had increased to approximately $140{ }^{\circ} \mathrm{C}$. An additional heating time of approximately $15 \mathrm{~min}$ increased the substrate temperature by another $10^{\circ} \mathrm{C}$. During a typical deposition of a CoCr film $200 \mathrm{~nm}$ thick it increased by $10^{\circ} \mathrm{C}$. At the start of deposition of the $T_{\mathrm{R}}$ samples the substrate temperature was approximately $25^{\circ} \mathrm{C}$. Considering these values with respect to a pure Co film, it follows that the ratio of substrate temperature to melting temperature of Co was low: 0.17 for the $T_{R}$ samples and below 0.27 for the $T_{H}$ samples. In Table 2 a number of $T_{\mathrm{s}} / T_{\mathrm{m}}$ quotients are given for the metals used.

The film thickness of our investigated films varied between 50 and $1580 \mathrm{~nm}$; the thicker films were mainly used as an aid to understanding the growth mechanism. The end pressure of the system was better than $10^{-4} \mathrm{~Pa}$. Typical pressures during deposition were $5 \times 10^{-6}-1 \times 10^{-4} \mathrm{~Pa}$ (depending on the substrate temperature). Before deposition most of the Si substrates were cleaned by boiling them for approximately $5 \mathrm{~min}$ in 2-propanol ("mosselectpur" grade). This was done in order to improve the adhesion of the layers to the substrate. These standard IC wafers are known to have a Si-oxide layer on the $\mathrm{Si}$ 
surface (see, for example, ref. 82). The polyimide substrates were mounted in a metal ring and put under tension on the substrate holder. The compositions of both the co-evaporated and single source samples were measured by X-ray fluorescence (XRF).

As compared to the single-source obliquely evaporated films, in the obliquely coevaporated films the $\mathrm{Co}$ and $\mathrm{X}$ adatoms have a directed component in two opposing directions. At the relatively low-mobility situations in our samples, this will more easily lead to elongated shapes with their long axes parallel to the incidence plane than in single-source obliquely deposited films with similar mobility conditions: compare Section 4.2. (Based on the random walk theory in ref. 83, values for the diffusion length in $\mathrm{Co}-\mathrm{Cr}$ are calculated ranging from $10 \mathrm{~nm}$ at $T_{\mathrm{s}}=200{ }^{\circ} \mathrm{C}$ to $170 \mathrm{~nm}$ at $T_{\mathrm{s}}=400{ }^{\circ} \mathrm{C}$.)

\subsection{Morphology}

It was discussed in Section 4 how an oblique-incident flux induces anisotropic morphological characteristics. By using two (or more) oblique-incidence fluxes the morphology can also become anisotropic. In fact the second (or further added) flux direction(s) are additional parameters by which the morphology is influenced and can be tailored. In this section we will show the existence of an anisotropic morphology in obliquely co-evaporated thin films for magnetic recording applications. Experimental data for several samples will be discussed. These include different metals as well as different deposition geometries (different angles of incidence).

Shadowing effects and surface diffusion greatly influence the growth of the films. The shadowing mechanism effectively means that atoms in the growing film shadow unoccupied sites from the direct path of incident atoms. Owing to the limited mobility, most of the unoccupied sites are left empty. The resulting morphology is columnar with the columns tilted towards the effective incoming direction: see, for instance, co-evaporated $\mathrm{Co}-\mathrm{Cr}$ films [2-5]. With the effective incoming direction getting closer to perpendicular incidence and/ or with increasing substrate temperature (and hence higher surface temperature and therefore more diffusion), the columns become more perpendicular.

In the case of the $M$ samples the direction of column inclination is the Co-source direction and for the $\mathrm{L}$ samples this is the $\mathrm{Cr}$ direction. The $\mathrm{S}$ samples have their columns inclined towards the (only present) source direction. Typical columnar tilt angles $(\beta$, measured with respect to the film normal) for the low-temperature samples $\left(T_{\mathrm{R}}\right)$ with $18-30$ at.\% $\mathrm{Cr}$ are: $12-15^{\circ}(\mathrm{M}), 10-11^{\circ}(\mathrm{L}), 0^{\circ}(\mathrm{LR})$ and $16^{\circ}(\mathrm{S})$. The single-source samples (S series) have the largest columnar tilt, which is not surprising since their effective incidence angle is the largest one, i.e. $27.5^{\circ}$. For the $M$ and $L$ series, the effective incidence angle is a weighted average of the two separate incidence angles: e.g. $75 \%$ of $\alpha_{i \text { co }}$ less $25 \%$ of $\alpha_{i \text { Cr}}$. Thus for the $M$ series an effective incidence angle smaller than $27.5^{\circ}$ results, and for the $L$ series an even smaller one is present because of the perpendicular incoming Co vapour (roughly speaking: $25 \%$ of $46^{\circ}$, being $11.5^{\circ}(\mathrm{L})$, is smaller than $(75 \%-25 \%)$ of $27.5^{\circ}$, being $13.75^{\circ}(\mathrm{M})$ ). Thus $\beta$ was expected to be smaller for the $\mathrm{L}$ series than for the $M$ series, which in turn was expected to be smaller than for the $S$ series. In the LR samples the rotation of the substrate during deposition averages out the incidence angle to zero and thus a zero tilt angle of the columns results.

The columnar-tilt angle decreases with the deposition temperature. Typical values for $\beta$ of the $T_{H}$ samples are: $7-10^{\circ}(\mathrm{M})$ and $2-3^{\circ}(\mathrm{S})$. The free energy of the columnar boundaries is proportional to the surface area of the boundaries, and is thus minimal if the columns 
grow perpendicular to the substrate. A higher deposition temperature provides more surface diffusion (partly cancelling the shadowing effect) and thus enables an energetically more favourable tilt angle of the columns, which is more perpendicular.

A number of the above mentioned features can be seen in Fig. 28. Here, a crosssectional SEM observation is given. Clearly observable is the tilted columnar structure. This sample easily broke along the column boundaries and we got the impression that its morphology was rather porous. In addition, in this SEM observation the slanting of the column tops can be seen.

In the incidence plane the columns are separated by open regions. In the transverse direction, where the vapour supply is homogeneous without shadowing effects, clustering of columns (bundling) can occur under certain deposition conditions. In the investigated $\mathrm{Co}-\mathrm{Cr}$ films, bundles are made most pronounced by the combination of opposite oblique $\left(27.5^{\circ}\right)$ directions of $\mathrm{Co}$ and $\mathrm{Cr}$, ambient substrate temperature and relatively low evaporation rate $\left(4 \AA \mathrm{s}^{-1}\right)$. At higher substrate temperatures more hexagon-like columns developed. At a higher deposition rate $\left(8-9 \AA \mathrm{s}^{-1}\right)$ the symmetrically co-evaporated Co-Cr films have elongated columns in the longitudinal direction. In the latter case the elongated crystallites have their long axis parallel and their short axis perpendicular to the incidence plane. Apparently the rate of newly arriving atoms with respect to the mean free path and the chance of trapping at the film surface (by, among other things, oxidation) was thus optimal for enhancing the particle growth in the longitudinal direction (pronounced adatom momentum parallel to the incidence plane). The combination of a relatively low adatom mobility in these samples and the opposing sides where the metals arrive yields, more easily in our opinion, elongated shapes with their long axes parallel to the incidence plane than is the case in single-source oblique deposition (compare Section 4.2).

A clear example of elongated crystallites with their long axis parallel to the incidence plane is given in the TEM planar-view observations in Fig. 29. This Co-Cr sample was coevaporated at a rate of $8.0 \AA \mathrm{s}^{-1}$. Co-Cr samples evaporated at the lower rates of approximately $4 \AA \mathrm{s}^{-1}$ showed bundles. This anisotropic morphological feature can be seen in Fig. 30, which shows SEM micrographs of the film surfaces of co-evaporated $\mathrm{Co}-\mathrm{Cr}$ films [4]. In Fig. 30(a) the tops of the columns are the rounded shapes, several of which cluster together in the transverse direction and form the bundles. In the direction parallel to the incidence plane the columns are separated by open regions (darkest parts in Fig. 30(a)) which are

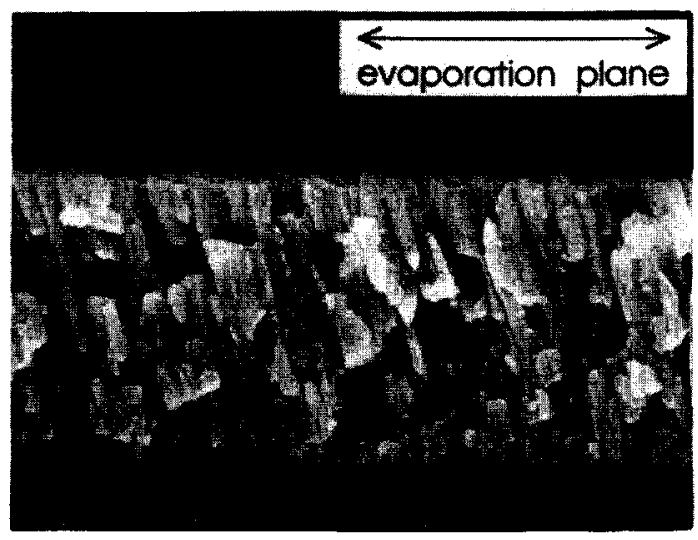

Fig. 28. SEM observation of a co-evaporated high-temperature $\mathrm{Co}_{80} \mathrm{Cr}_{20}$ sample $1.58 \mu \mathrm{m}$ thick. The cross-section is parallel to the incidence plane [1]. 
a)

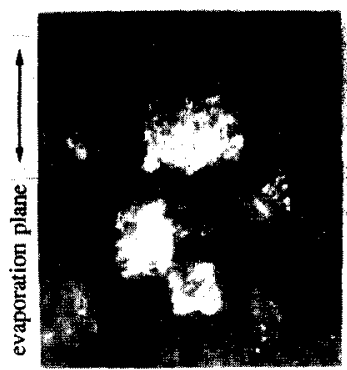

b)

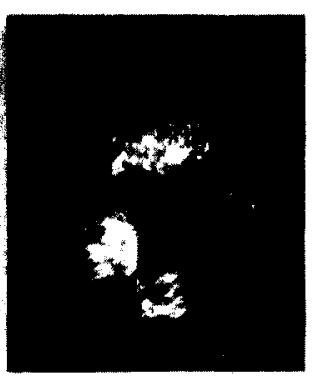

Fig. 29. TEM planar-view observations of a co-evaporated high-temperature $\mathrm{CO}_{79} \mathrm{Cr}_{21}$ sample $1.35 \mu \mathrm{m}$ thick: (a) brightfield image, (b) dark-field image. The sample was slightly etched, by which the Co-rich parts were preferentially dissolved and the $\mathrm{Cr}$-rich parts were passivated. The incidence plane is approximately parallel to the arrow [1].

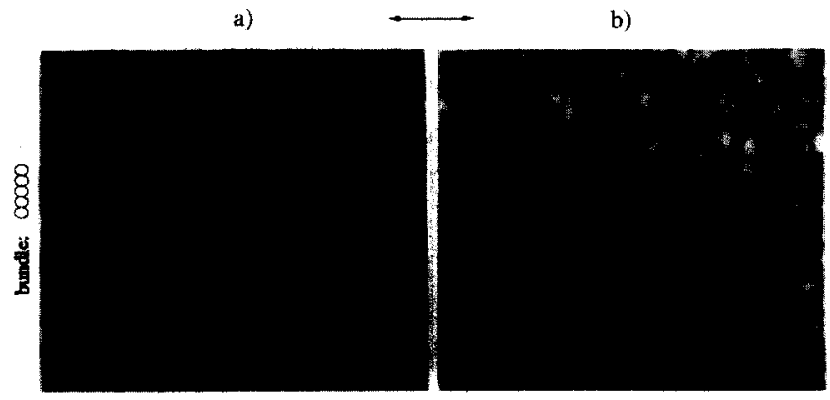

Fig. 30. Surface SEM observations of co-evaporated Co-Cr films approximately $700 \mathrm{~nm}$ thick, deposited at different substrate temperatures [4]. (a) $T_{\mathrm{R}}$ sample with clearly visible bundles (columnar aggregates perpendicular to the incidence plane). (b) $T_{\mathrm{H}}$ sample having both elongated shapes and hexagonal shapes (e.g. at the arrow) present.

caused by the shadowing mechanism. Perpendicular to the incidence plane this shadowing is not present. In this direction there is a homogeneous arrival of vapour material and thus there are no (large) separations between the columns. All our co-evaporated $\mathrm{Co}-\mathrm{Cr} T_{\mathrm{R}}$ samples on Si deposited at $4 \AA \mathrm{s}^{-1}$ exhibit these features; in thinner samples the sizes are smaller, which is related to the smaller columnar diameter in the thinner films. The packing density for these samples therefore is highest in the transverse direction. Our $T_{\mathbf{H}}$ samples (film thicknesses less than $800 \mathrm{~nm}$ ) show different surface structures with the development of hexagon-like columns (see Fig. 30(b), e.g. at arrow), and bundles cannot be distinguished clearly. The largest columnar sizes belong to hexagon-shaped columns. These shapes were found not only in our $T_{\mathrm{H}}$ samples deposited at $4 \AA \mathrm{s}^{-1}$ but also in those deposited at 9 $\AA \mathrm{s}$ found (and $h<800 \mathrm{~nm}$ ). Compared with the $T_{\mathrm{R}}$ samples, the $T_{\mathrm{H}}$ samples have a higher density, compare Figs. 30(a) and 30(b).

The density difference also follows from the comparison of measured film thicknesses with X-ray fluorescence (XRF) and a step-profilometer (DEKTAK). The film thicknesses as determined by XRF and DEKTAK step-profiling measurements were in good agreement with each other for the high-temperature samples $\mathrm{Co}-\mathrm{Cr}\left(\mathrm{M}-T_{\mathrm{H}}\right)$ and for the Co-Cr samples that were co-evaporated with rotating substrates (referred to as LR samples). This indicates that in these films the mass thickness approximately equals the geometric thickness, and that the volume packing of $\mathrm{Co}$ and $\mathrm{Cr}$ material is high. Since in the XRF calculations a weighted average of the densities of pure $\mathrm{Co}$ and pure $\mathrm{Cr}$ is used, this comparison is hampered by not knowing the densities of $\mathrm{Co}-\mathrm{Cr}$ samples which are in a compositionally separated state. Another inaccuracy arises with the open regions between columns and the 
surface roughness. Both increase the measured XRF intensities by which the XRF film thicknesses are determined to be too large. The $\mathrm{Co}-\mathrm{Cr}$ samples co-evaporated at low temperature $\left(\mathrm{M}-T_{\mathrm{R}}\right)$ have a larger geometric thickness than mass thickness. This indicates that these samples have a relatively low packing density, which no doubt will be caused by open regions that exist between the columns due to the shadowing effect. A higher deposition temperature (and thus higher surface diffusion), as well as rotation of the substrate during evaporation, partly cancels the shadowing effect and can thus lead to denser structures. The ratio of mass to geometric film thicknesses (XRF to DEKTAK) is 0.93-0.94 in the $\mathrm{M}-T_{\mathrm{R}}$ samples with film thicknesses between 240 and $700 \mathrm{~nm}(22-26$ at.\% $\mathrm{Cr}$, average) and in the $\mathrm{L}-T_{\mathrm{R}}$ samples it increases with decreasing $\mathrm{Cr}$ content from around 0.95 for 30 at.\% $\mathrm{Cr}$ to around 0.98 for 15 at.\% $\mathrm{Cr}$. Its dependence on the $\mathrm{Cr}$ content in the $\mathrm{L}$ samples was expected since the $\mathrm{Cr}$ vapour is the only obliquely arriving vapour flux in these samples. With less $\mathrm{Cr}$ the amount of normally arriving (Co) vapour is larger, which yields fewer and/or narrower open regions between the columns (less shadowing during growth).

The porous columnar structure was also present in co-evaporated $\mathrm{CO}_{45} \mathrm{Ag}_{55}$ films (Fig. 31(a)). The columns are tilted through $10-11^{\circ}$ towards the Ag source direction. This direction is obviously the result of the larger atomic percentage of $\mathrm{Ag}$ than of $\mathrm{Co}$. In co-evaporated $\mathrm{Co}_{77} \mathrm{Ta}_{23}$ (Fig. 31(b)) a very fine columnar structure exists with the columns tilted $6^{\circ}$ towards the Co-source direction. In neither the $\mathrm{Co}-\mathrm{Ag}$ nor the $\mathrm{Co}-\mathrm{Ta}$ films was aggregation or elongation of the columns found. However, the small columnar dimensions (and low contrasts) will not easily enable the observation of these scrutinized features. The packing density in the low-temperature samples is low. This of course is a result of the shadowing mechanism during film growth.

Obliquely co-evaporated $\mathrm{Fe}\left(\alpha_{\mathrm{i}}=60^{\circ}\right)$ also consists of a porous columnar structure with aggregates of columns in the transverse direction (bundles) [84]. Here the model is presented as reproduced in Fig. 32. With normal incidence deposition a perpendicular columnar morphology develops. With single-source oblique-incidence deposition the columnar morphology is tilted towards the incoming vapour-beam direction, and columns aggregate in

a)

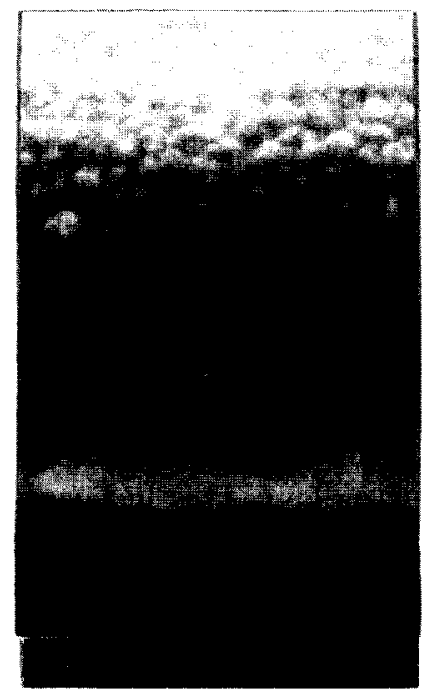

b)

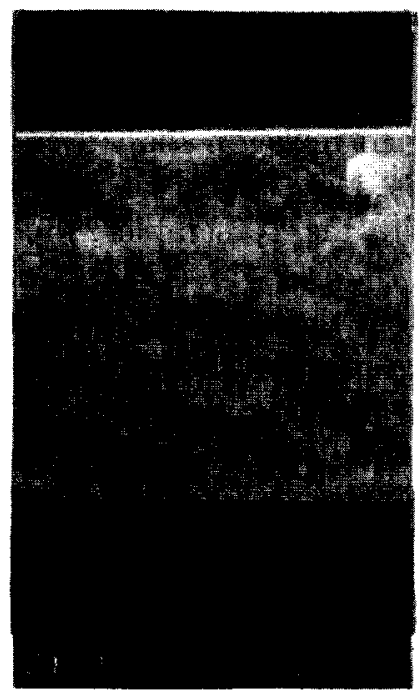

Fig. 31. Cross-sections parallel to incidence plane of co-evaporated (a) $\mathrm{Co}-\mathrm{Ag}$ and (b) $\mathrm{Co}-\mathrm{Ta}$ [1]. 


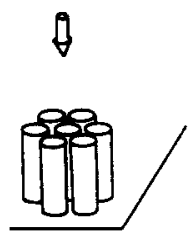

(a)

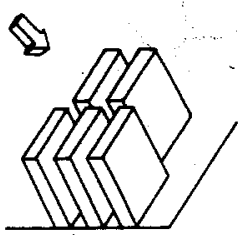

(b)

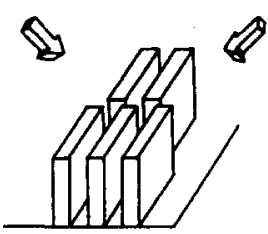

(c)

Fig. 32. Models for columnar morphologies: (a) normal-incidence films; (b) oblique-incidence film from one source; (c) oblique-incidence film from two opposite sources [84].

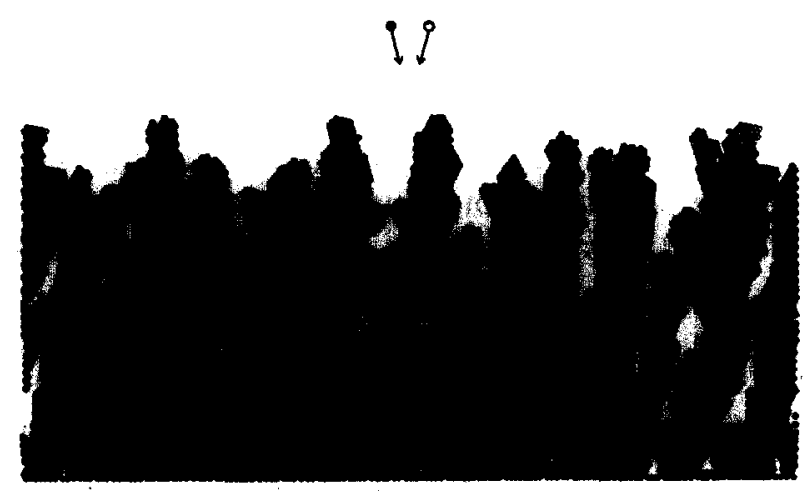

(a)

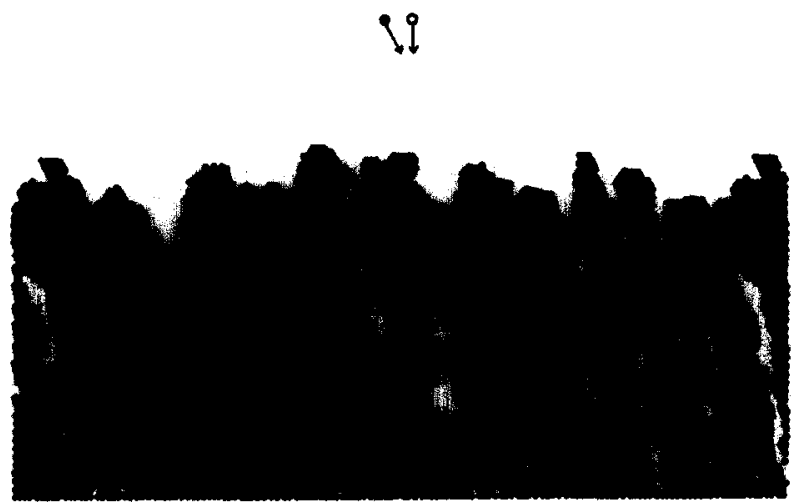

(b)

Fig. 33. Simulated $\mathrm{Co}_{78} \mathrm{Cr}_{22}$ structures from two sources at low diffusion $\left(d_{1}=73\right.$ ): (a) $\alpha_{\mathrm{iCo}}=\alpha_{\mathrm{iCr}}=15^{\circ}$ and (b) $\alpha_{\mathrm{iCo}}=0^{\circ}$ and $\alpha_{i} c_{r}=27.5^{\circ}$. The solid circles represent the $\mathrm{Cr}$ atoms, the open ones the Co atoms [1].

the transverse direction. With dual-source oblique-incidence deposition where the two incidence angles are equal (and opposite in sign) a perpendicular columnar structure develops that also exhibits bundles.

Our simulations of film growth confirm the development of a columnar structure that inclines towards the effective incoming vapour direction. Figure 33 shows some examples of this. Since the simulated structures involve $\mathrm{Co}_{78} \mathrm{Cr}_{22}$, in the geometry with symmetric vapour fluxes (Fig. 33(a)) the effective incoming direction is tilted towards the incoming Co direction. In the geometry with perpendicular incoming Co vapour (Fig. 33(b)) the effective incoming direction is tilted towards the incoming $\mathrm{Cr}$ vapour. The inclination angle of the columnar-like features in the simulated structures increased with the incidence angle for every fixed surface diffusion. Further, with increasing surface diffusion the inclination 
angle of the column-like structures decreased and their widths increased. At the same time the film structures became denser (the porosity of the morphology could clearly be observed in the simulated figures).

The comparison of columnar-tilt angles for the single- and dual-source geometries is also in agreement with the experimental results. Compared to the single-source simulations, the average inclination angle of the column-like structures is smaller than in the dualsource case. This behaviour can be explained by an average angle of incoming atoms that is less than the incidence angle of the single-source simulations. Since the simulations were only two-dimensional, the anisotropic column aggregation was not seen in the simulated morphologies.

The adatom mobility in the $\mathrm{Co}-\mathrm{Cr}$ and $\mathrm{Co}-\mathrm{Ag}$ films deposited at ambient substrate temperatures ( $T_{\mathrm{R}}$ films) was probably of limited value, because a crystalline structure had formed. If the adatoms had no mobility at all, the structure could have become a "frozen state" resulting in an amorphous structure [85]. It has also been shown $[26,28]$ that a crystalline structure can result from athermal crystallization processes, see Section 3 . In contrast to the $\mathrm{Co}-\mathrm{Cr}$ and $\mathrm{Co}-\mathrm{Ag}$ films, the higher melting temperature of $\mathrm{Ta}$ is expected to largely prohibit the adatom mobility in the $\mathrm{Co}$-Ta films. Almost no crystallinity could be measured for most Co-Ta films, and the films were close to amorphous. A poorly developed fcc polycrystalline structure could be measured in the thickest films.

\subsection{Local composition in the lateral direction}

A second feature, besides the morphology, that can be tailored by using two (or more) oblique vapour fluxes is the local chemical composition in the films. As in Section 5.2, this will be shown in examples of thin films for magnetic recording (and, incidentally, confirmed by films for optical applications). In thin films for magnetic recording, local regions with less- or non-ferromagnetic regions are desirable for a good magnetic performance. Tailoring the local composition in the lateral direction is thus a necessity, and if it can be done at low substrate temperatures there is an advantage, because in that case relatively cheap polyester substrates can be used for flexible media instead of the more expensive polyimide foils.

In our investigation we restricted ourselves to two vapour-flux directions. If one of the two vapours is dominant, it can determine, to a large extent, the growth of the thin film. As an example, obliquely co-evaporated $\mathrm{Co}-\mathrm{Cr}$ films have a dominating Co vapour $[2,4,6]$. In these films the Co vapour is responsible for most of the deposited atoms. Because of its oblique-incidence arrival at the film surface, atoms already deposited will shadow adjacent regions from direct impingement of more Co atoms, which leads, under low-mobility conditions, to inclined columnar growth towards the Co source. The columns will be separated from each other by open regions. This shadowing model of growth was already postulated by König [36] and Smith [10] and is described in more detail in Section 4. The Cr atoms, which form the minor evaporation flux, come from the opposite direction and thus have the shadowed area in the opposite direction to that of the Co. They are most likely to impinge on the Co shadowed-area side at every stage of the growth $[2,4]$. With limited mobility (especially with low-temperature deposition) the adatoms will not have sufficient energy to move around to find the energetically most favourable places. This results in socalled process-induced compositional separation $[2,4,6,86,87]$, in which one side of the columns is Cr-rich. Process-induced compositional separation is thus a geometry-induced chemical inhomogeneity. The growth mechanism just described is illustrated in Fig. 34 [2]. 


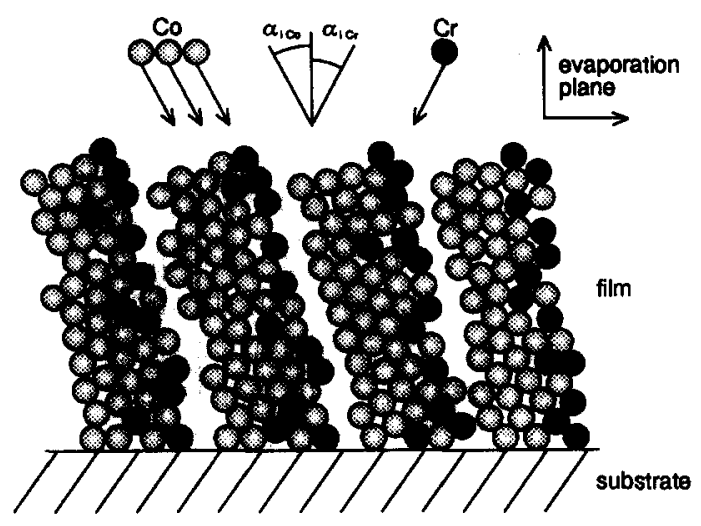

Fig. 34. Cross-sectional model for the morphology parallel to the incidence plane [2].

At higher substrate temperatures, more surface diffusion will take place and transition to the thermally enhanced compositional separation can occur. This thermally enhanced compositional separation is not restricted to the field of oblique co-deposition. In $\mathrm{Co}-\mathrm{Cr}$, phase separation [88] is predicted to occur $[89,90]$, possibly driven by magnetic (exchange) energy [83,91].

Since our main interest in this section is tailoring the local composition by using oblique incidence deposition, we will concentrate on results concerning process-induced compositional separation. Where necessary, this will be extended to thermally enhanced compositional separation, which is, as mentioned above, not a specific feature of oblique deposition.

In general, as indicator for the alloying behaviour of two metals, the so-called heat of formation can be used, which is formulated in the Miedema model [92]. Although Miedema's approach seems to be of limited value, we use it here as a rough indicator. The heat of formation is considered to be the dominant factor explaining why $\mathrm{Co}$ and $\mathrm{Ag}$ are nonalloying metals (Co and $\mathrm{Cr}$ are predicted to alloy). The Miedema model is a semi-empirical model based on calculation of the heat of formation for the alloying behaviour of two metals. Two parameters are essential: the difference in chemical potential of the two metals and the difference in density of electrons at the boundary of the atomic cells of the metals. The difference in chemical potential describes the energy transfer from cells with a relatively high chemical potential to cells with a lower chemical potential. This transfer reduces the energy of the system and therefore decreases the heat of formation. A discontinuity in the density of electrons in the alloy is considered to be disadvantageous and is therefore considered to increase the heat of formation. If the heat of formation is positive, then energy is needed to form the alloy, which makes it unlikely that many compounds exist. If the heat of formation is negative, then energy is freed upon alloying.

Miedema's calculations resulted in a fast graphical method (alloying diagram) to see if two metals will alloy or not. It appeared that $\mathrm{Co}$ will not alloy with $\mathrm{Ag}$ (nor with $\mathrm{Cu}$ or $\mathrm{Au}$ ). In the case of $\mathrm{Co}-\mathrm{Ta}$ the large differences in atomic radius and electronegativity are assumed to make alloying difficult [92]. Three different metal combinations are thus investigated, being $\mathrm{Co}$ and $\mathrm{Cr}$ (same radii, partly alloying, $\mathrm{CS}$ possible, promising and extensively studied material for high-density recording), $\mathrm{Co}$ and $\mathrm{Ag}$ (different radii, nonalloying, no expected electron sharing), and $\mathrm{Co}$ and $\mathrm{Ta}$ (different radii, partly alloying, electron sharing expected to be comparable to $\mathrm{Co}$ and $\mathrm{Cr}$, large melting point of $\mathrm{Ta}-$ 
less adatom mobility during growth). Comparison of these films may yield more understanding of the relation between growth, microstructure and magnetic behaviour.

If we extend our 2-D model of process-induced compositional separation (Fig. 34) to three dimensions, Fig. 35 results. The geometric effect yields two different shadowed directions, one for each of the materials of each direction. The minor material flux is most likely to impinge on the shadowed-area side of the major material flux at every stage of film growth. As already explained with Fig. 34, the combination of shadowing, low adatom mobility and impingement sites can cause the enrichment of one component at one side of the columns. In this mechanism, the slanting of the tops of the columns plays a role because of the possible impingement sites. When the columns are tilted towards the direction of the major material, the most favourable situation arises. That is to say, in this case the column tops are slanted towards the direction of the major material. This enhances the probability that the minor material merely impinges on one side of the columns.

Thus, for instance, in the concrete cases of the co-evaporated $\mathrm{Co}-\mathrm{Cr}$ samples with symmetric geometry $\left(\mathrm{M}\right.$ series, $\alpha_{\mathrm{i} \mathrm{Co}_{0}} \approx \alpha_{\mathrm{i} \mathrm{Cr}} \approx 27.5^{\circ}$ ) and asymmetric geometry (L series, $\alpha_{\mathrm{i} \mathrm{Co}_{0}} \approx 0^{\circ}$, $\alpha_{\mathrm{i} \mathrm{C}_{\mathrm{r}}} \approx 46^{\circ}$ ) the slanting of the column tops is more favourable in the $M$ series than in the $\mathrm{L}$ series to provide enhanced impingement of $\mathrm{Cr}$ on the Co-shadowed side. With limited mobility (especially with the $T_{\mathrm{R}}$ samples evaporated at ambient substrate temperature), the adatoms will not have sufficient energy to move to the energetically most favourable places. The geometric effect will then dominate the distribution of the two materials and the socalled process-induced compositional separation is the result, which will be shown to exist in $\mathrm{Co}-\mathrm{Cr}$ with several characterization methods in this section. Of course with processinduced compositional separation there is not a very strict separation of the two components. There will be a gradual change since the minor component (in Figs. 34 and 35 represented by the solid circles) will also impinge at sites that are not located at the column boundaries. Besides this, the minor component will also diffuse to some extent inside the column. The more slanted the column tops are, the larger is the probability of the occurrence of processinduced compositional separation. We used several tools to check the state of compositional separation. We shall discuss them one by one in the following text.

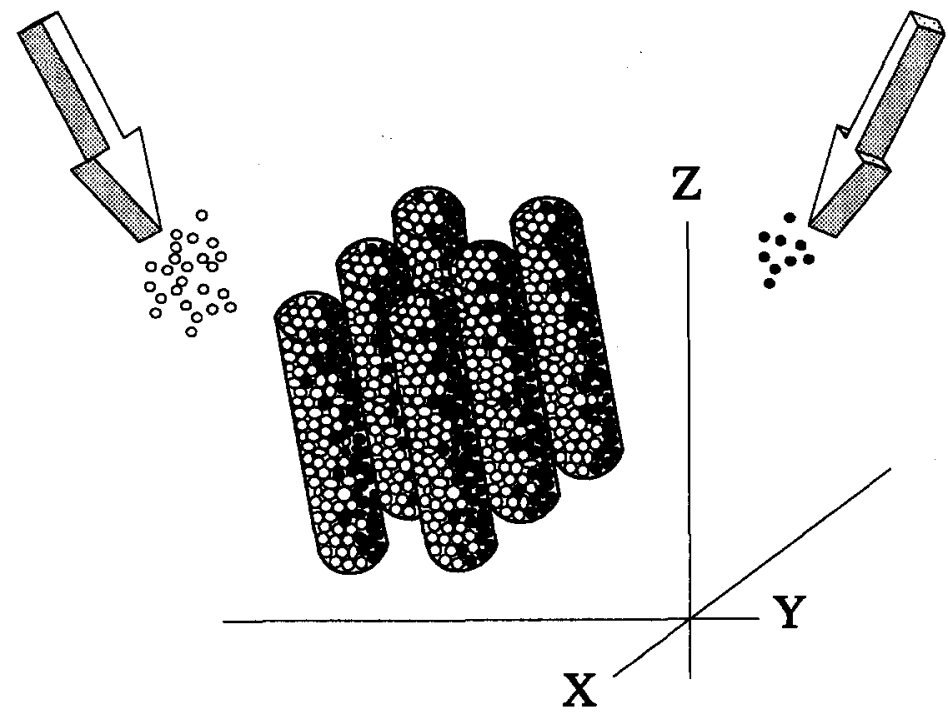

Fig. 35. 3-D model of process-induced compositional separation [1]. 


\subsubsection{Computer simulation of the microstructure}

By simulating the film growth we qualitatively predicted the process-induced compositional separation; see, for example, Fig. 36. In the simulation we used bonding energies for Co and $\mathrm{Cr}$ [6]. In the figure the $\mathrm{Co}$ is represented by the open symbols and $\mathrm{Cr}$ by the solid symbols. Clearly observable in the simulated figure is the enrichment of $\mathrm{Cr}$ at the Coshaded side of the columns. This separation of the two components is, in the case of Co and $\mathrm{Cr}$, stronger at low diffusion and is due to the fact that from energetically favourable aspects $\mathrm{Co}$ and $\mathrm{Cr}$ like to (partly) alloy. At higher (surface) diffusion more intermixing of Co and Cr occurs, as can be seen when comparing Figs. 36(a) and 36(b). With decreasing dual-incidence angles the degree of compositional separation decreased. In the case of process-induced compositional separation, which is, in fact, a geometry-induced effect that occurs, it is not necessary to have equal opposing incidence angles. Figure 37 shows two examples where the incidence angles of $\mathrm{Co}$ and $\mathrm{Cr}$ are unequal. With a greater difference between the two incoming directions, the degree of separation is larger. It follows from Fig. 37(a) that with the Co atoms impinging normally $\left(\alpha_{\mathrm{i} \mathrm{Co}}=0^{\circ}\right)$ and the $\mathrm{Cr}$ atoms obliquely $\left(\alpha_{\mathrm{i} C \mathrm{r}} \neq 0^{\circ}\right)$ the column-like structures are slightly tilted towards the $\mathrm{Cr}$ source direction. This is in agreement with the experimental findings for the samples of the $\mathrm{L}$ series. If the Co incidence flux is slightly tilted in the opposite direction to the $\mathrm{Cr}$ incidence flux, it acts in a compensating manner for the resulting tilt angle of the column-like structures; compare Figs. 37(a) and 37(b). Obviously the combination of many Co atoms (in this case, 78 at.\%) impinging under a slight angle $\left(10^{\circ}\right)$ and fewer $\mathrm{Cr}$ atoms (22 at.\%) impinging under a larger but opposing angle $\left(27.5^{\circ}\right)$, counterbalance each other so that the column-like structures are oriented (almost) perpendicular. As may be expected, the degree of surface diffusion also plays a role in this.

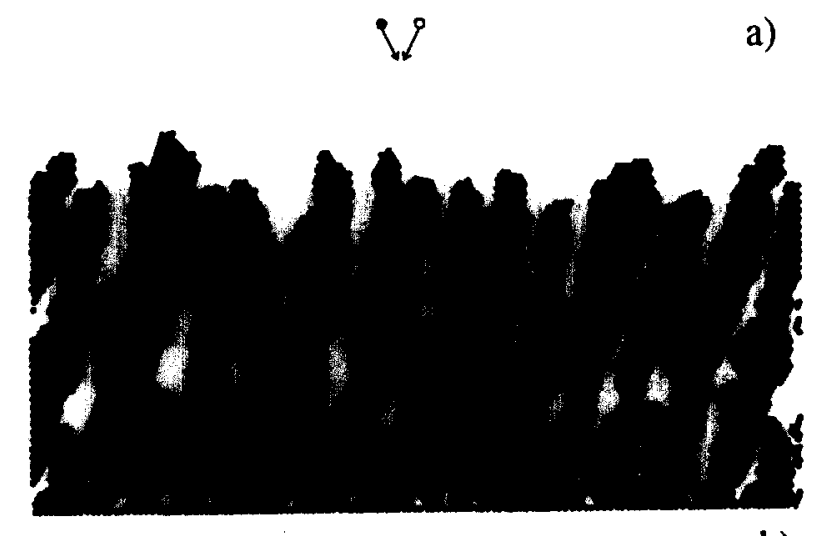

a)

b)

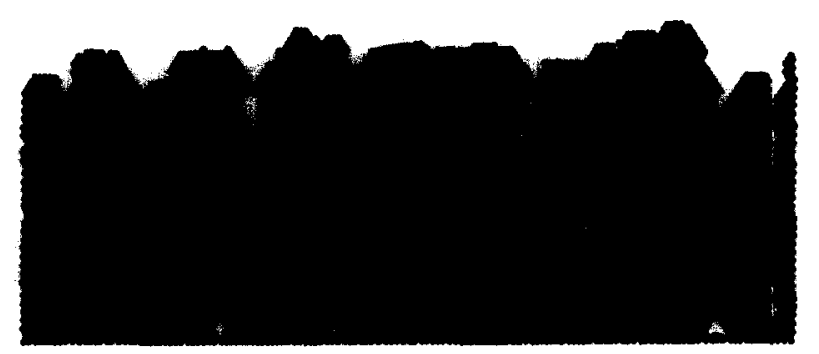

Fig. 36. Simulated $\mathrm{Co}_{78} \mathrm{Cr}_{22}$ structures from two sources with $\alpha_{i c_{0}}=\alpha_{i c r}=27.5^{\circ}$ at low ((a) $\left.d_{1}=73\right)$ and high ((b), $d_{1}=2336$ ) surface diffusion. The solid circles represent the $\mathrm{Cr}$ atoms, the open ones the Co atoms [4]. 
li

a)

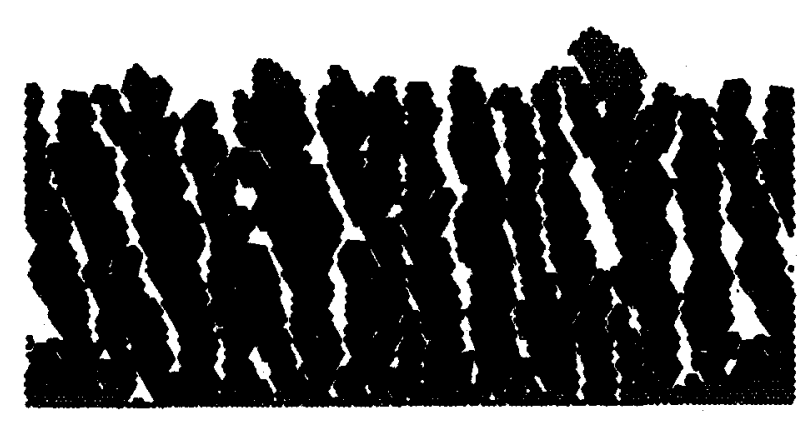

iv

b)

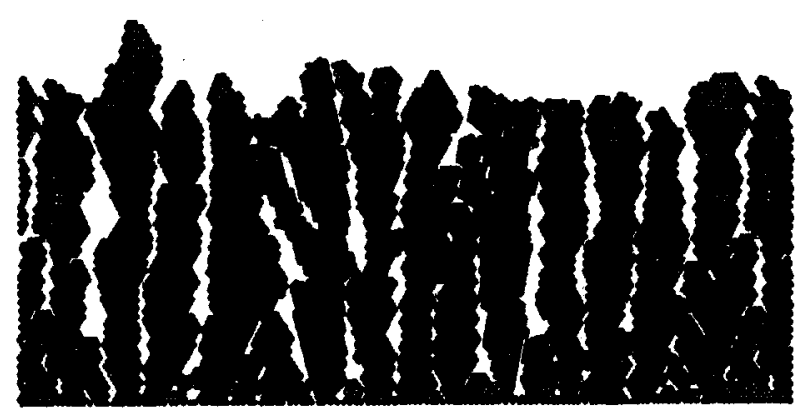

Fig. 37. Simulated $\mathrm{CO}_{78} \mathrm{Cr}_{22}$ structures from two sources at low surface diffusion $\left(d_{1}=73\right)$. The solid circles represent the $\mathrm{Cr}$ atoms, the open ones the Co atoms. (a) $\alpha_{i C_{0}}=0^{\circ}, \alpha_{i C_{r}}=45^{\circ}$; (b) $\alpha_{i C_{0}}=-10^{\circ}, \alpha_{i C_{r}}=27.5^{\circ}[1]$.

Other reports made (including simulations of film growth) of (expected) inhomogeneous distributions in low temperature co-evaporated materials concern $\mathrm{Fe}-\mathrm{Cu}$ [93], $\mathrm{Fe}-\mathrm{Al}$ and $\mathrm{Fe}-\mathrm{Cr}$ [94] and Ag-SiO2 [95-97]. Also an earlier publication by Leamy and Dirks [98] reported on a simulation result concerning oblique deposition from two separate sources that had one species heavily concentrated on one side of each column.

\subsubsection{AES analysis of the fractured surface}

By the use of Auger electron spectroscopy (AES), direct proof was established of the existence of process-induced compositional separation in co-evaporated $\mathrm{Co}-\mathrm{Cr}$ [6]. In order to do this, a fractured room-temperature sample was observed by AES in a plane perpendicular to the evaporation plane, see Fig. 38. On both (as-broken) surfaces the $\mathrm{Co}, \mathrm{Cr}$ and $\mathrm{O}$ Auger signals were measured, in total at five different areas. Figure 38 gives the measured (original) signals for one area of each surface. On one surface (" $A$ ") a clear Cr signal was measured, while on the other surface (" $B$ ") only a slight signal was detected, as can be seen in Fig. 38. Further, the Co signal was less strong on the A side than on the $B$ side. Hence, direct proof established with AES can be added to the indirect proof of the existence of process-induced compositional separation, as discussed earlier and later on again in this section. It can therefore be concluded that compositional separation can be induced by two opposing directions of ferromagnetic and non-ferromagnetic vapour. 

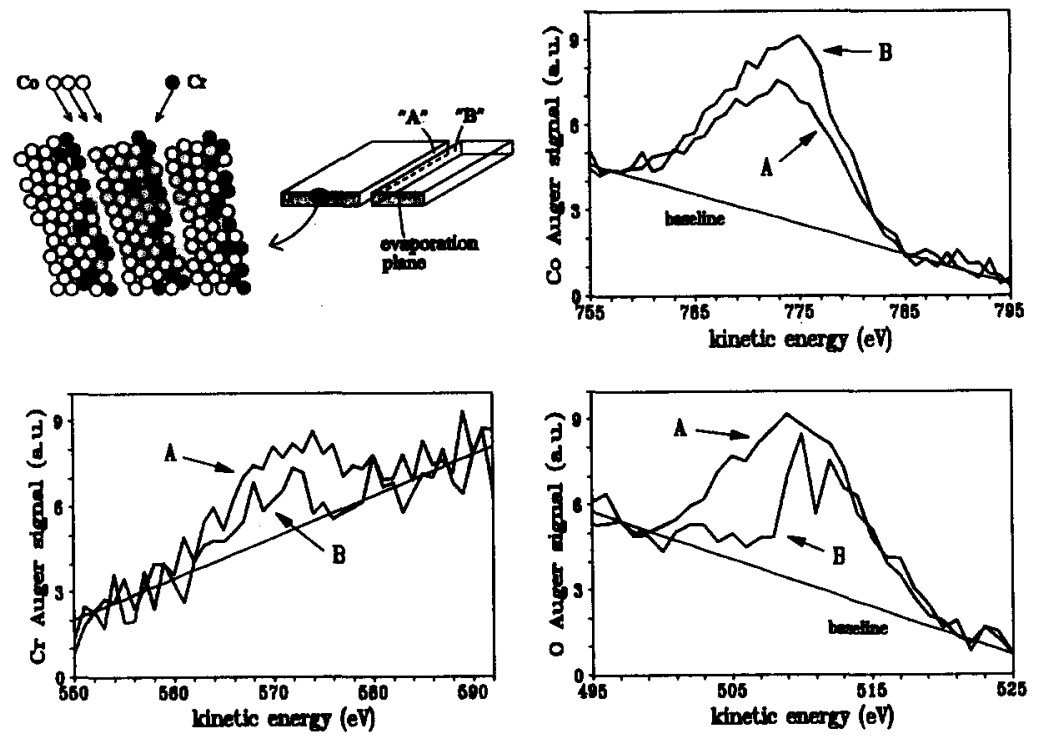

Fig. 38. $\mathrm{Co}, \mathrm{Cr}$ and $\mathrm{O}$ Auger signals measured on the Cr-rich ("A") and Co-rich ("B") sides of a $\mathrm{CO}_{79} \mathrm{Cr}_{21} \mathrm{sample}$ of $367 \mathrm{~nm}$ thickness co-evaporated at low temperature [6].

Quantitization of these Auger spectra is difficult, because the $\mathrm{Cr}$ peak is extremely low. Smoothing and differentiating can falsely raise the calculated amount of $\mathrm{Cr}$, so caution is necessary and the original data, as presented in Fig. 38, are the most reliable measure. However, an attempt was still made to quantitize, both by directly taking an 11-point and a five-point differential of the energy spectrum $(N(E) \times E)$. Standard factors are taken for $10 \mathrm{kV}$ as sensitivity factors: $\mathrm{Cr}, 0.1476$; $\mathrm{Co}, 0.19 ; \mathrm{O}, 0.350$. The $\mathrm{Cr}$ peak around $570-575$ $\mathrm{eV}$ was scanned, because with this peak less overlap occurs with the oxygen peak than is the case with the main $\mathrm{Cr}$ peak. The Co percentage was normalized to the total of $\mathrm{Co}+\mathrm{Cr}+\mathrm{O}$. On the A side of the sample (two different measurement areas) the Co (relative) percentage was around $41-44 \%$. On the B side of the sample (three different measurement areas) the Co (relative) percentage was around $50-56 \%$. Since oxygen mainly alloys with $\mathrm{Cr}$ and not with $\mathrm{Co}$, and because the amount of $\mathrm{Cr}$ is almost unmeasurable, the $\mathrm{Co}$ percentage is thought to be the most reliable quantitative measure. Again the conclusion is that the A side is the Co-poor (and thus the Cr-rich) surface and that the B side is the Co-rich (and thus the Cr-poor) surface.

\subsubsection{NMR measurements}

We have established proof, also by indirect measurements, of the occurrence of compositional separation. The temperature for the low-temperature samples ( $T_{\mathrm{R}}$ samples) was far too low to produce thermally enhanced compositional separation, and thus another mechanism must have induced the separation, being what we called process-induced compositional separation. For indirect measurement results, we will discuss the spin-echo spectra from nuclear magnetic resonance (NMR) measurements and the energy product as measured with the vibrating-sample magnetometer (VSM). We will not describe (results of) other methods that can be used to determine the state of compositional separation, such as, for instance, selective chemical etching in combination with TEM observations and energy dispersive X-ray analysis (EDX) measurements [2,99], EDX high-spatial-resolution 
X-ray micro-analysis (using the scanning mode of TEM) [100,101] or atom-probe field-ion microscopy (FIM), which has only recently been applied in the investigations on compositional separation $[102,103]$.

NMR analysis was performed on the following samples: co-evaporated $\mathrm{Co}-\mathrm{Cr}$ (both on $\mathrm{Si}-\mathrm{M}$ and $\mathrm{L}$ series - and polyimide substrates), single source $\mathrm{Co}-\mathrm{Cr}$, obliquely evaporated Co and co-evaporated Co-Ag and Co-Ta. The NMR spectrum of the pure Co film, Fig. 39 , shows a large resonance peak around $220 \mathrm{MHz}$. This resonance originates from pure Co regions. Figure 40 shows the spin-echo spectrum of an $\mathrm{L}$ film $200 \mathrm{~nm}$ thick with 8 at.\% Cr. Besides the main line around $220 \mathrm{MHz}$, this spectrum contains a clear (first) satellite peak around $175 \mathrm{MHz}$. Again the main line is due to a highly pure Co component. The first satellite peak is due to an amount of Co surrounded by one $\mathrm{Cr}$ nearest neighbour [104-106]. The appearance of a strong main line and the first satellite peak justify the conclusion that in this sample compositional separation has obviously occurred, and that highly pure Co regions are present. This can only be caused by the process geometry, because the deposition temperature, being room temperature, is far too low to cause thermally enhanced compositional separation. Also for samples from the other series, NMR analysis supports the existence of highly separated regions. Figs. 41-44 show measured spin-echo spectra for low and high temperature $M$ and $S$ samples. It follows from the NMR measurements (including comparison of the spectra in Figs. 41-44) that:

- All these samples contain separated regions of highly pure Co components. They have, however, various stages of compositional separation.

- With increasing average $\mathrm{Cr}$ content, the resonance frequency of the main line decreases and the width of the peak increases. Thus the amount of $\mathrm{Cr}$ in the highly pure Co

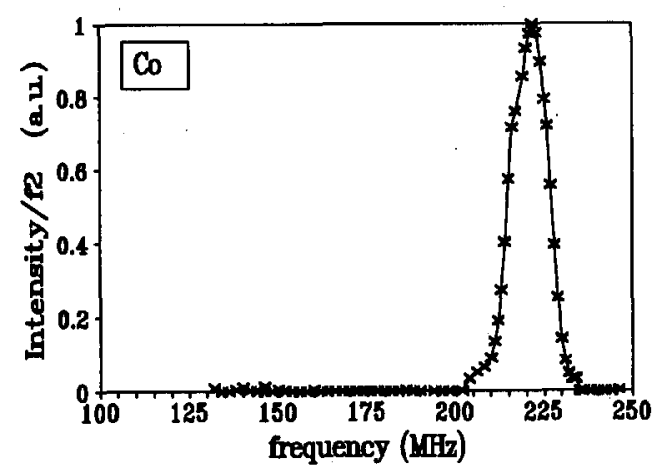

Fig. 39. Spin-echo spectrum of obliquely evaporated Co $\left(\alpha_{i}=27.5^{\circ}, T_{\mathrm{R}}, h=180 \mathrm{~nm}\right)[1]$.

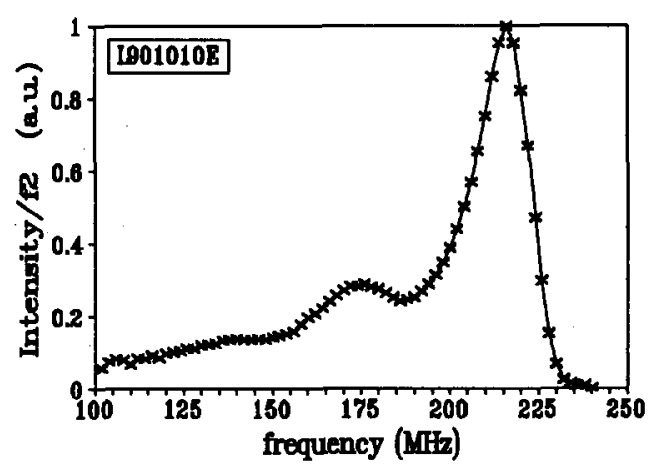

Fig. 40. Spin-echo spectrum of obliquely co-evaporated $\mathrm{CO}_{92} \mathrm{Cr}_{8}$ from the $\mathrm{L}$ series $\left(T_{\mathrm{R}}, h=180 \mathrm{~nm}\right)$ [1]. 


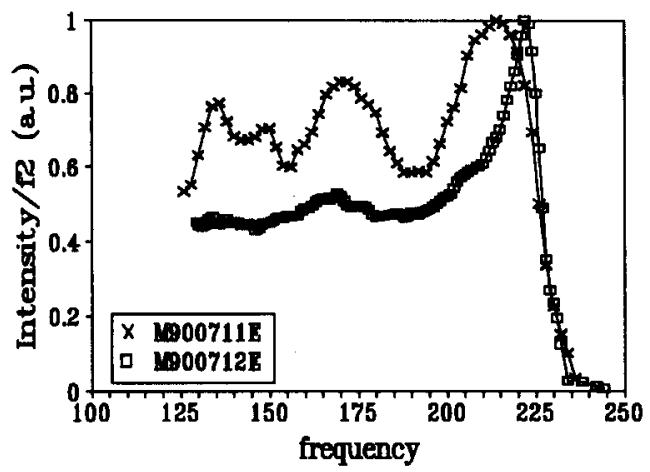

Fig. 41. Spin-echo spectrum of obliquely co-evaporated $T_{\mathrm{R}}$ films from the $\mathrm{M}$ series: $\mathrm{M} 900711 \mathrm{E}\left(\mathrm{Co}_{76} \mathrm{Cr}_{24} ; h=231 \mathrm{~nm}\right)$ and $\mathrm{M} 900712 \mathrm{E}\left(\mathrm{CO}_{75} \mathrm{Cr}_{25}, h=356 \mathrm{~nm}\right)[1]$.

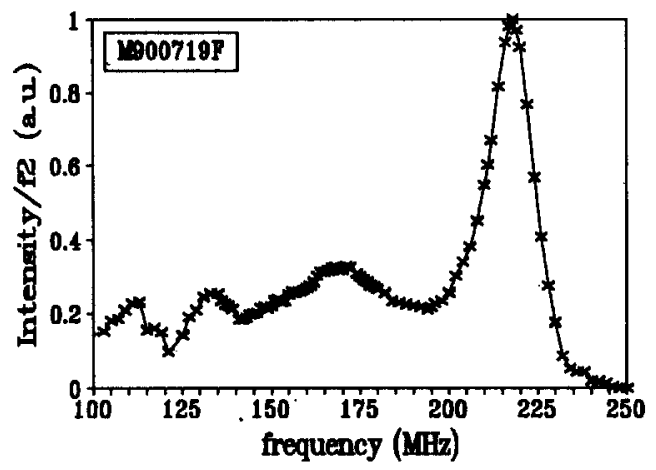

Fig. 42. Spin-echo spectrum of obliquely co-evaporated $T_{\mathrm{H}}$ films from the $\mathrm{M}$ series: $\mathrm{M900719F}\left(\mathrm{Co}_{75} \mathrm{Cr}_{25}, h=267 \mathrm{~nm}\right)$ [1].

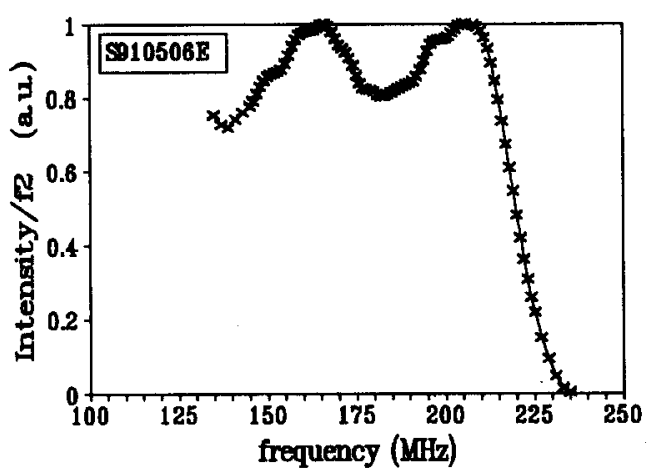

Fig. 43. Spin-echo spectrum of obliquely co-evaporated $T_{\mathrm{R}}$ films from the $\mathrm{S}$ series: $\mathrm{S910506 \textrm {E }}\left(\mathrm{CO}_{82} \mathrm{Cr}_{18}, h=227 \mathrm{~nm}\right)$ [106].

component increases with the average $\mathrm{Cr}$ content. Further, with increasing average $\mathrm{Cr}$ content, the first satellite peak increases its intensity with respect to the main line. This means that the regions where $\mathrm{Co}$ is surrounded by one or more $\mathrm{Cr}$ nearest neighbours increase.

- With increasing film thickness the main line becomes relatively stronger and the satellite lines become relatively weaker. This indicates that the volume part of highly pure Co regions increases with film thickness. 


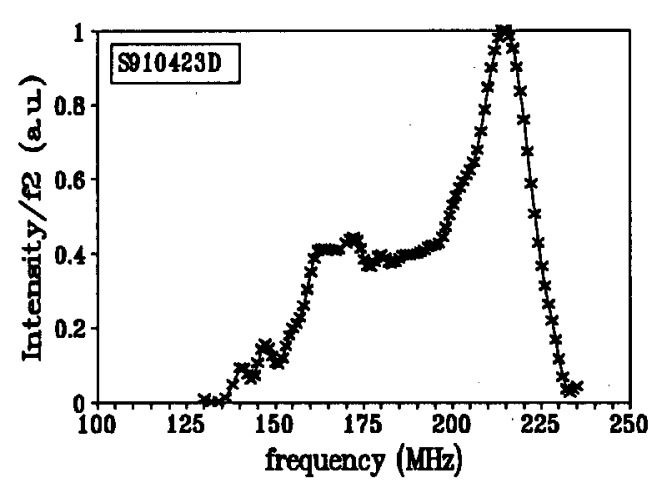

Fig. 44. Spin-echo spectrum of obliquely co-evaporated $T_{\mathrm{H}}$ films from the S series: $\$ 910423 \mathrm{D}\left(\mathrm{Co}_{75} \mathrm{Cr}_{25}, h=207 \mathrm{~nm}\right)$ [106].

- The high-temperature samples have relatively stronger main lines and relatively weaker satellite lines than the low-temperature samples (Figs. 41-44). Hence, the compositionally separated state of the $T_{\mathrm{H}}$ samples consists of relatively more highly pure Co regions and fewer regions where $\mathrm{Co}$ is surrounded by one or more $\mathrm{Cr}$ nearest neighbours.

- The resonance frequency of the main line for the single-source samples is higher for the $T_{\mathrm{H}}$ films than for the $T_{\mathrm{R}}$ films (e.g. Figs. 43-44). Therefore the amount of $\mathrm{Cr}$ in the highly pure Co component is lowest in the $T_{H}$ films. The same holds true for the comparison between the $T_{\mathrm{R}}$ and $T_{\mathrm{H}}$ films for the 250-nm-thick co-evaporated samples (Figs. 41-42). The reverse is true for the co-evaporated Co-Cr films of 400 and $700 \mathrm{~nm}$ thickness, i.e. the highly pure Co regions of these $T_{\mathrm{R}}$ samples contain a smaller $\mathrm{Cr}$ content.

- The $\mathrm{Cr}$ content in the highly pure Co regions is smaller (higher resonance frequency of the main line) for the co-evaporated Co-Cr samples than for the single-source evaporated Co-Cr samples. This indicates a higher compositionally separated state in the co-evaporated films, which can be attributed to process-induced compositional separation.

- The co-evaporated samples have relatively stronger main and weaker satellite lines than the single-source samples. (Thus the volume part of the highly pure Co components is largest for the co-evaporated samples.)

Drawing conclusions from the intensity of the main line with respect to that of the satellite lines, we can say that with increasing film thicknesses, with increasing deposition temperatures and with evaporation from two sources instead of one the volume part of highly pure Co regions in the Co-Cr films increases. Drawing conclusions from the resonance frequencies of the main lines, we can say that the co-evaporated films have a higher compositionally separated state than the single-source samples.

Clearly the NMR measurement results have proved that highly pure Co regions are present in the co-evaporated $T_{R}$ samples. Since the deposition of these films was carried out with a substrate temperature below $50^{\circ} \mathrm{C}$, this indirectly proves the presence of processinduced compositional separation. The substrate temperature is, in other words, far too low for the occurrence of thermally enhanced compositional separation. In addition, the higher compositionally separated state in the co-evaporated films with respect to the singlesource samples is also attributed to process-induced compositional separation.

The NMR spectrum of a $\mathrm{Co}_{42} \mathrm{Ag}_{58}$ film approximately $200 \mathrm{~nm}$ thick contained a resonance peak around $215 \mathrm{MHz}$. Thus compositional separation has occurred in the sample. The spin-echo signal was weak, and a large amount of noise was present. This was probably the result of the small Co volume in the sample. It seemed there was also a small satellite 
peak, which must have arisen from regions in which the hyperfine field of Co is lowered. This would imply that there is not a complete and full separation of Co and Ag. After annealing at successive steps of 225,325 and $425{ }^{\circ} \mathrm{C}$, each for two and a half hours, the compositionally separated state developed even more, with only a resonance peak from a highly pure Co component being present. This peak seemed to consist of an hcp domain signal (at $220.5 \mathrm{MHz}$ ) and an hep wall signal (at $225.5 \mathrm{MHz}$ ).

The NMR spin-echo intensity for a $\mathrm{Co}_{77} \mathrm{Ta}_{23}$ film (350 nm thick) monotonically decreased as the frequency increased from 140 to $240 \mathrm{MHz}$. This very much resembled the spectrum of a homogeneous $\mathrm{Co}-\mathrm{Cr}$ alloy; see, for example, ref. 104. The Co-Ta film thus appeared to be quite homogeneous.

\subsection{4. (Magnetic) energy product}

The second indirect-measurement result used as indication for the occurrence of processinduced compositional separation is the (magnetic) energy product (product of coercivity and saturation magnetization) as obtained from hysteresis loops that are measured with a VSM. Figure 45 shows coercivity values as a function of saturation magnetization for Co$\mathrm{Cr}$ samples. The perpendicular coercivities obey the previously measured relation of reaching a maximum at a certain saturation magnetization [107], see Fig. 45. In the lower $M_{\mathrm{s}}$ region an increase in $H_{\mathrm{c} \perp}$ with increasing $M_{\mathrm{s}}$ is possibly the result of a local spread in magnetization. In the higher $M_{\mathrm{s}}$ region the decrease in $H_{\mathrm{c} \perp}$ with increasing $M_{\mathrm{s}}$ can be caused by a larger exchange (narrower non-ferromagnetic regions) between adjacent columns. Other researchers have also found such a behaviour of $H_{c \perp}$ as a function of $M_{\mathrm{s}}$ [108-110]. Striking are the enhanced coercivity values for the (symmetric) co-evaporated samples (M, F series) with respect to the single-source samples (S series). The discontinuities in the obliquely evaporated films, i.e. the open (and low density) regions between the columns that are formed owing to shadowing and the $\mathrm{Cr}$ enhancement at the columnar boundaries that arises due to process-induced compositional separation (i.e. in the co-evaporated films) hinder domainwall movement by acting as pinning centres, thereby leading to enhanced perpendicular coercivity. The smaller expected discontinuities in the rotated $\mathrm{Co}-\mathrm{Cr}$ samples (LR series)

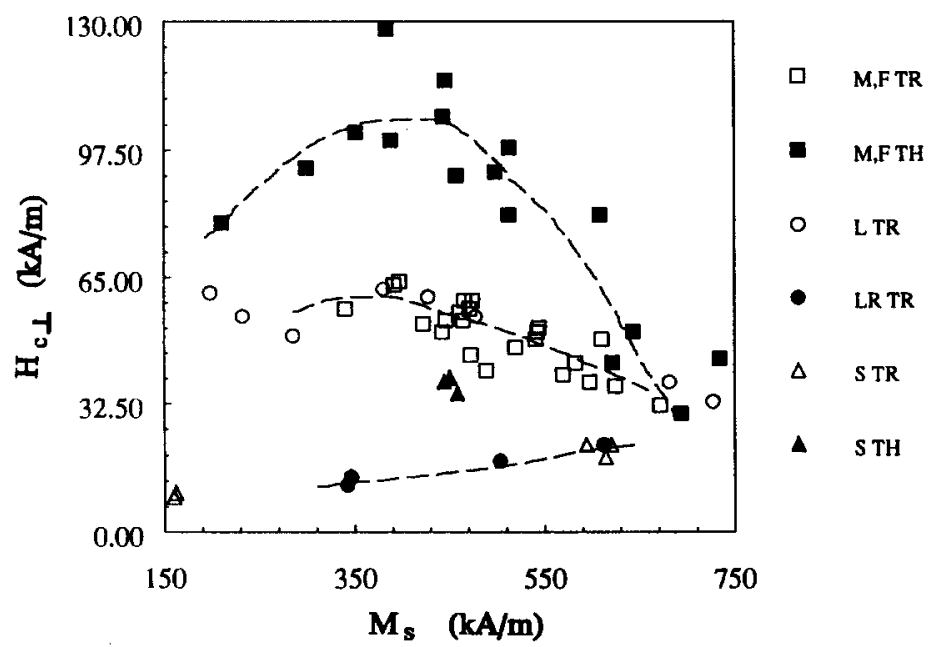

Fig. 45. Perpendicular coercivity as a function of the saturation magnetization for our (co-)evaporated Co-Cr films [1]. 
are one of the origins of its lower $H_{\mathrm{c} \perp}$ as compared to the other $\mathrm{Co}-\mathrm{Cr}$ samples. Rotating at a higher speed will decrease the size of the discontinuities (open regions) even more, and thus further lower the coercivity. A second probable origin is a possibly more homogeneous state in the LR samples (see text below).

The influence of the columnar size on the large perpendicular coercivity is limited in the (co-evaporated $T_{R}$ ) Co-Cr films. The dimensions in the 200-250 nm-thick films are close to each other. In samples of the $\mathbf{M}$ and $\mathbf{F}$ series, columnar sizes of approximately $12.5-25$ $\mathrm{nm}$ were measured. The sizes are smaller for both the $\mathrm{L}$ and $\mathrm{LR}$ samples, namely less than $10 \mathrm{~nm}$. However, they are, on the average, smaller in the LR than in the L films, but the difference is only small. Since the perpendicular coercivities are comparable in the $\mathrm{M}$ and $\mathrm{L}$ films, while in the LR films $H_{\mathrm{c} \perp}$ is much smaller, this definitely rules out the columnar size as predominant for a large $H_{\mathrm{c} \perp}$. The $\mathrm{Co}-\mathrm{Ag}$ and $\mathrm{Co}-\mathrm{Ta}$ films have very low perpendicular coercivities. The main reason for this is believed to be the small effective (uniaxial) anisotropy constant. Further, small columnar sizes are not advantageous (because of this the discontinuities are also small and thus the reduction in magnetostatic interaction between neighbouring columns is less effective). It is known from the literature that Co$\mathrm{Cr}-\mathrm{Ta}$ has a low coercivity. For instance, in sputtered films an excess of Ta addition (more than 5 at.\%) caused the degradation of $H_{c \perp}$ [111].

5.3.4.1. Saturation magnetizations for the co-evaporated $\mathrm{Co}-\mathrm{Cr}$ films. In the case of the low-temperature samples, we attribute the enhanced coercivities to process-induced compositional separation. In principle, this cannot be proved by the extrinsic coercive field. A better parameter for this is the saturation magnetization $M_{\mathrm{s}}$ as a function of the average chemical content. In $\mathrm{Co}-\mathrm{X}$ alloys, where the magnetic moment of $\mathrm{Co}$ can normally be reduced by the filling of its $3 \mathrm{~d}$ shell and reduction of spin-exchange energy (less Co-Co neighbours), the effect of compositional separation is a rise in saturation magnetization. The highly pure Co regions in a compositionally separated structure have high magnetic moments and the macroscopic (and average) saturation magnetization is thus enhanced as compared with homogeneous alloys. All co-evaporated samples of the $\mathrm{Co}$ - $\mathrm{Cr}$ films have such an enhancement of $M_{\mathrm{s}}$; see Fig. 46, where a comparison can be made with "homogeneous bulk" Co-Cr layers, of which the best-known approximation is given by the cross symbols. These "bulky" Co-Cr alloys are prepared in an arc melting furnace [112]. Co-Cr powder samples are shown to have approximately the same $M_{\mathrm{s}}(\% \mathrm{Cr})$ values [113].

Data for two ribbons from ref. 114 are also given in Fig. 46 for comparison. According to NMR measurements [106] these ribbons are in a more homogeneous state than the Co$\mathrm{Cr}$ powder samples. The model lines are for random distributions of $\mathrm{Co}$ and $\mathrm{Cr}$ and no $\mathrm{Cr}-\mathrm{Cr}$ bonds, according to models from Haines [115] and Fartash et al. [116]. The differences between these two models are two-fold. The first difference concerns the assumption about how much each additional $\mathrm{Cr}$ atom at a nearest-neighbour site of $\mathrm{Co}$ reduces the magnetic moment of $\mathrm{Co}$. Haines assumed that each $\mathrm{Cr}$ atom contributed equally. Fartash found, by fitting to experimental data on $\mathrm{Co}-\mathrm{Cr}$ alloys, that the first $\mathrm{Cr}$ atom at a nearest-neighbour site decreases the magnetic moment of $\mathrm{Co}$ the most, and that each additional $\mathrm{Cr}$ atom contributes less. The second difference concerns the average atomic percentage of $\mathrm{Cr}$ where $M_{\mathrm{s}}$ becomes zero. Both use the experimental data that $M_{\mathrm{s}}=0$ for an average $\mathrm{Cr}$ content of 25 at.\%. This is more or less the case for $\mathrm{Co}-\mathrm{Cr}$ samples in a "homogeneous state". Fartash projects this to the model with a random distribution, while Haines chooses the model with no $\mathrm{Cr}-\mathrm{Cr}$ bonds. In principle the latter yields a real homogeneous distribution. 


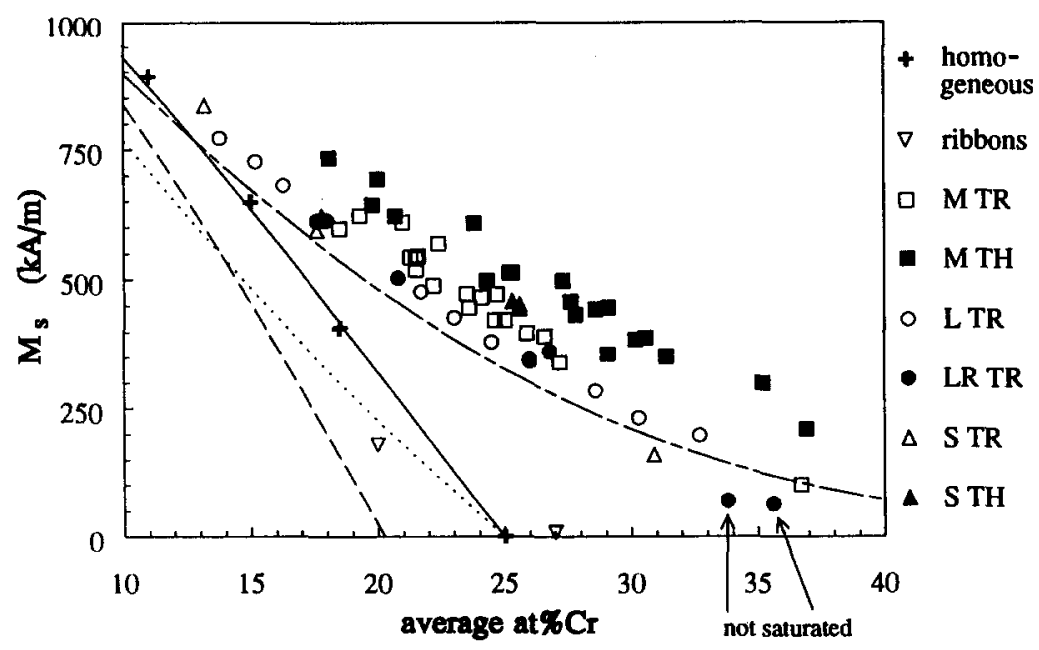

Fig. 46. Saturation magnetization as a function of the average $\mathrm{Cr}$ content for several $\mathrm{CoCr}$ samples [1]. The supposedly homogeneous $\mathrm{Co}-\mathrm{Cr}$ alloys are from ref. 112. The lines are from the following models: the random-distribution model (centre dashed from ref. 115 and solid from ref. 116) and the model with no $\mathrm{Cr}-\mathrm{Cr}$ bonds (dotted from ref. 115 and dashed from ref. 116).

The question then arises about how homogeneous the films from the experimental data really are. Most likely they are less homogeneous than in the case of no $\mathrm{Cr}-\mathrm{Cr}$ bonds. For instance, quenched $\mathrm{Co}-\mathrm{Cr}$ ribbons from ref. 114 have lower $M_{s}$ values than the data used, and from NMR measurements [106] they are known to be much more homogeneous than powder samples which had approximately the same $M_{s}(\% \mathrm{Cr})$ values as the data used from ref. 112. The data points for two ribbons are added in Fig. 46. Assuming the ribbons are truly homogeneous, it can be concluded that the model lines of Haines decrease too slowly, while those of Fartash decrease too quickly.

Both the co-evaporated $T_{\mathrm{R}}$ samples and the $T_{\mathrm{H}}$ samples have an enhanced saturation magnetization and, as explained above, the large $M_{\mathrm{s}}$ values originate from highly pure Co regions. A separation into $\mathrm{Co}$-rich and $\mathrm{Cr}$-rich regions can occur in the low-temperature samples owing to the special geometry of the opposing vapour beams. In the $T_{\mathrm{R}}$ samples this process-induced compositional separation, in which one side of the columns is Cr-rich, is believed to be the main origin of the enhanced saturation magnetization (and energy product), since the substrate temperature is very low. A rise in temperature of $10^{\circ} \mathrm{C}$ was measured during evaporation of a film $200 \mathrm{~nm}$ thick by means of a thermocouple in contact with the back of the substrate. More surface diffusion will have taken place in the $T_{H}$ samples during film growth owing to the preheated substrate. Thus a combination of processinduced and thermally enhanced compositional separation is likely to have occurred. Thereby the state of compositional separation has apparently increased, since the $M_{\mathrm{s}}$ values are larger than those of the $T_{R}$ samples.

The enhancement of $M_{\mathrm{s}}$ is larger for the $M$ series than for the $L$ series, implying that the $\mathrm{L}$ films are more homogeneous (compare data in Fig. 47, where error bars of 5\% are included). The expected higher degree of slanting of the column tops in the $M$ samples as discussed earlier is thought to be the main reason for the higher degree of processinduced compositional separation in the $\mathrm{M}$ films. The rotated asymmetric $\mathrm{Co}-\mathrm{Cr}$ samples have similar $M_{\mathrm{s}}(\% \mathrm{Cr})$ values to the non-rotated asymmetric ones, which indicates that their states of inhomogeneity are comparable as far as the Co-rich regions are involved. This, 


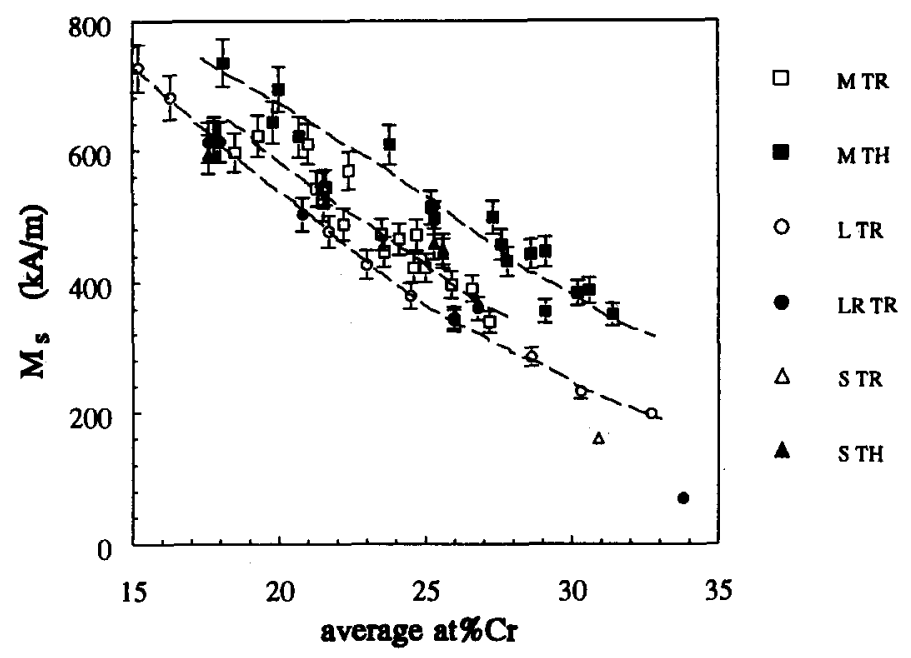

Fig. 47. Saturation magnetization as a function of the average $\mathrm{Cr}$ content for several $\mathrm{Co}-\mathrm{Cr}$ samples with error bars of $5 \%[1]$.

however, is only true if the ferromagnetic volume parts are equal, because in a highly compositionally separated sample with Co-Co clusters and $\mathrm{Cr}-\mathrm{Cr}$ clusters the "correct" saturation magnetization of the ferromagnetic volume part is obtained by excluding the non-ferromagnetic volume. Doing this raises the values of $M_{s}(\% \mathrm{Cr})$.

In a more homogeneous sample the (total mass-)volume used is closer to the real ferromagnetic volume. Assuming the LR films are more homogeneous than the L films, and since the total mass-volumes are used in Fig. 46 for both series, it is then possible that the saturation magnetizations are similar for equal average $\mathrm{Cr}$ content, although the state of inhomogeneity may differ. It might be expected from the growth that there is a more homogeneous distribution of $\mathrm{Cr}$ and $\mathrm{Co}$ in the rotated (LR) films. At every stage of film growth, $\mathrm{Cr}$ and $\mathrm{Co}$ arrive at the film surface at the same height of film development. What is actually required is that the Co is ahead of the Cr, so that first the Co forms the columns, after which the $\mathrm{Cr}$ impinges on the columnar boundaries and not on the column tops. The curving of the column tops is important. Since in the LR films the effective incoming vapour direction is perpendicular to the substrate, the column tops are not likely to be slanted towards one direction. This will not encourage process-induced separation of $\mathrm{Cr}$ and $\mathrm{Co}$ [3].

The Co-Cr samples evaporated obliquely from one alloy source ( $\mathrm{S}$ series) tend to have the most homogeneous $\mathrm{Co}$ and $\mathrm{Cr}$ distributions of all investigated evaporated $\mathrm{Co}-\mathrm{Cr}$ films (the trend of the saturation magnetization values of the $S$ series is below the trend of the $\mathrm{M}$ series). The comparison between these $\mathrm{S}$ samples and the other $\mathrm{Co}-\mathrm{Cr}$ films is probably the most reliable, since they were evaporated in the same equipment under conditions (both of deposition and characterization) that can be compared in detail. Such a comparison is more difficult with samples deposited and investigated by others. In order to realize this, one only needs to think of the surface temperature of the growing film surface, which is of vital importance for the normally occurring thermally enhanced compositional separation. Matters like substrate-holder temperature contact and measurement of substrate temperature need to be similar to make a good comparison possible.

If one does try to compare the data with other data, such as, for instance, for sputtered $\mathrm{Co}-\mathrm{Cr}$, it follows that the $\mathrm{M}_{\mathrm{s}}(\% \mathrm{Cr})$ is much higher for the evaporated $\mathrm{Co}-\mathrm{Cr}$ samples. For 
example, sputtered $\mathrm{Co}-\mathrm{Cr}$ films from Geerts et al. [117] have saturation magnetizations of $440 \mathrm{kA} \mathrm{m}^{-1}$ with an average $\mathrm{Cr}$ content of 19.5 at.\%, $371 \mathrm{kA} \mathrm{m}^{-1}$ with 20.5 at.\% $\mathrm{Cr}$ and $240 \mathrm{kA} \mathrm{m}^{-1}$ with 24 at.\% Cr; compare this with Fig. 46. Sputtering was done for these films on Si substrates with the same orientations as were used in the evaporation, and the substrate holder was water-cooled. During evaporation the substrate temperature, measured by a thermocouple at the back of the $\mathrm{Si}$, increased by $10^{\circ}$ (film thickness, $200 \mathrm{~nm}$ ). With the starting temperature being room temperature, co-evaporated $\mathrm{Co}_{80} \mathrm{Cr}_{20}$ had $M_{\mathrm{s}}$ values around $600 \mathrm{kA} \mathrm{m}^{-1}$ and $\mathrm{Co}_{76} \mathrm{Cr}_{24}$ around $460 \mathrm{kA} \mathrm{m}^{-1} . M_{\mathrm{s}}$ of the single-source samples is also higher than that of sputtered films. This can be correlated with differences in local Co-Cr distributions, differences in film densities that should be used in the XRF data and differences in surface temperatures and deposition rates.

Other researchers also found a higher $M_{\mathrm{s}}(\% \mathrm{Cr})$ for evaporated than sputtered Co-Cr; see, for example, ref. 118 , where $\mathrm{Co}-\mathrm{Cr}$ was co-evaporated under opposing angles, both of $7.4^{\circ}$, on rotated polyimide foils (50 rev $\min ^{-1}$; evaporation rate, $2 \mathrm{~nm} \mathrm{~s}^{-1}$ ). Simulations of film growth under two opposing angles of $7.5^{\circ}$ also show process-induced compositional separation, although this is less pronounced than under incidence angles of $27.5^{\circ}$. The enhanced $M_{\mathrm{s}}(\% \mathrm{Cr})$ values in the films from ref. 118 are therefore thought to have their origin in process-induced compositional separation. Their values are comparable to the ones found for the $M$ series and larger than for the LR series. The higher deposition rate used in ref. 118 probably resulted in a higher surface temperature of the growing film than was the case in the films of Fig. 46. Not much more can be said about it, since there is no information available about the film surface temperature (conduction between the $50 \mu \mathrm{m}$ thick polyimide substrates and substrate holder and the temperature contact between them).

In the case of the $S$ samples, a clear enhancement of $M_{\mathrm{s}}(\% \mathrm{Cr})$ occurs if the substrate is preheated $\left(T_{\mathrm{H}}\right.$ samples, $\left.T_{\mathrm{p}}=400^{\circ} \mathrm{C}\right)$. This is attributed to thermally enhanced compositional separation, similar to that of sputtered $\mathrm{Co}-\mathrm{Cr}$ films [119]. The enhancement of the saturation magnetization of the co-evaporated high-temperature $\mathrm{Co}-\mathrm{Cr}$ films (also with $T_{\mathrm{p}}=400{ }^{\circ} \mathrm{C}$ ) with respect to the $T_{R}$ samples is likely to arise from a change of process-induced compositional separation to thermally-enhanced compositional separation. The NMR measurements support the higher inhomogeneous state of the $T_{\mathrm{H}}$ films.

5.3.4.2. Saturation magnetizations for the co-evaporated Co-Ta films. Process-induced compositional separation is also present in the $\mathrm{Co}-\mathrm{Ag}$ and $\mathrm{Co}-\mathrm{Ta}$ films, although the degree of occurrence is different for $\mathrm{Co}-\mathrm{Ag}, \mathrm{Co}-\mathrm{Ta}$ and $\mathrm{Co}-\mathrm{Cr}$. In particular, the change of $M_{\mathrm{s}}$ upon annealing indicates this. Saturation magnetizations for co-evaporated Co-Ta values are given as a function of the average Ta content in Fig. 48. It can be seen that the saturation magnetization decreases with average $\mathrm{Ta}$ content in a similar way to that found for the symmetrically co-evaporated $\mathrm{Co}-\mathrm{Cr} T_{\mathrm{R}}$ samples. The $M_{\mathrm{s}}(\% \mathrm{X})$ data is however lower for Co-Ta than for Co-Cr. It can be concluded from the strong decrease that certainly no agreement is found with the phase diagram of Co-Ta (see ref. 120, for example) which is attained under equilibrium conditions. The decrease of $M_{\mathrm{s}}$ with increasing Ta content is much too strong for only a dilution effect between 5 and 33 at.\% Ta as expected from the phase diagram. This can be concluded by correcting for a non-magnetic volume part, since, if it were true, the only influence the Ta atoms would have on the magnetic moment of the Co atoms (between 5 and 33 at.\% $\mathrm{Ta}$ ) is that of lowering the exchange energy by reducing the number of Co-Co neighbours. This should give a linear decrease of $M_{\mathrm{s}}($ at.\%) equal to a line through 0 at. $\%, 1422 \mathrm{kA} \mathrm{m}^{-1}$ and 80 at. $\%, 0 \mathrm{kA} \mathrm{m}^{-1}$. Figure 48 gives the 


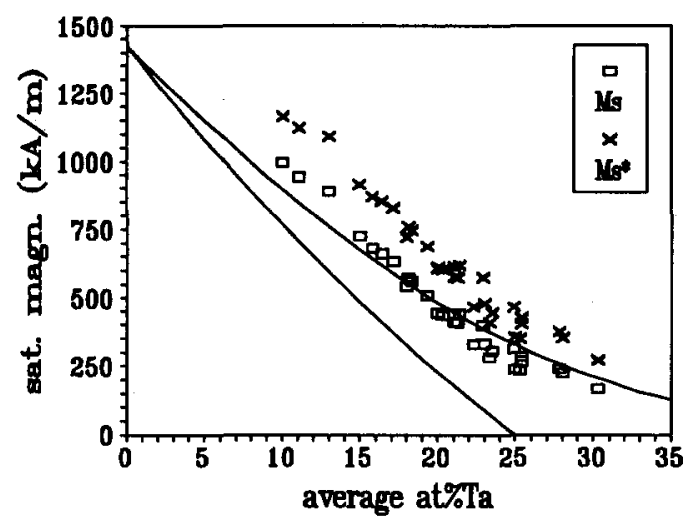

Fig. 48. Saturation magnetization as a function of the average Ta content for co-evaporated Co-Ta films. The solid lines are the model lines for $\mathrm{Co}-\mathrm{Cr}$ (from ref. 115) and are drawn for comparison [1].

saturation magnetization as a function of average Ta content, both normal and corrected values. Clearly, the corrected $M_{\mathrm{s}}$ values strongly decrease in the investigated Ta region of 10-30 at.\%, and this decrease is much stronger than can be expected for dilution alone. Thus the magnetic moment of $\mathrm{Co}$ is reduced by filling its $3 \mathrm{~d}$ shell with electrons supplied by Ta. A zero $M_{\mathrm{s}}$ with approximately $24-27$ at.\% Ta was found for sputtered Co-Ta [121-123], while 25 at.\% $\mathrm{Cr}$ is mostly found for sputtered $\mathrm{Co}-\mathrm{Cr}$ [112].

These data compared with Figs. 48 and 46 yield the conclusions that the co-evaporated Co-Ta films are more inhomogeneous than sputtered Co-Ta (thus some influence of the geometry effect of opposing vapour beams is present) and that probably less electrons of $\mathrm{Ta}$ than $\mathrm{Cr}$ are transferred to the unfilled $3 \mathrm{~d}$ shell of $\mathrm{Co}$ in the $\mathrm{Co}-\mathrm{Ta}$ and $\mathrm{Co}-\mathrm{Cr}$ alloys. Annealing at $325{ }^{\circ} \mathrm{C}$ decreased the saturation magnetization of $\mathrm{Co}_{70} \mathrm{Ta}_{30}$ and, to a much lesser degree, also that of $\mathrm{Co}_{80} \mathrm{Ta}_{20}$. This indicates that either homogenization or oxidation occurred.

Thus, to summarize: $M_{\mathrm{s}}$ in co-evaporated Co-Ta films is higher than for sputtered CoTa; after annealing $M_{\mathrm{s}}$ decreases and, owing to the geometry effect, a small degree of compositional separation is expected for the partly alloying Co-Ta. However, with the NMR measurements no Co-rich areas could be measured. The arrangement of Ta atoms is expected to be more or less "frozen" at the impingement spot (owing to the high melting point of $\mathrm{Ta}$ ). Furthermore, the columnar diameter is much smaller than in $\mathrm{Co}-\mathrm{Cr}$ (which is in agreement with the lower $T_{\mathrm{s}} / T_{\mathrm{m}}$ of Co-Ta). The columnar size is smaller than $10 \mathrm{~nm}$ in Co-Ta. Thus one columnar diameter usually contains fewer than 10 atoms of Ta and less than 30 atoms of Co. The small columnar size and the as-frozen positions of Ta with little or no relaxation to nearby positions probably yield Co-rich and non-ferromagnetic areas that are too small for even the process geometry to induce clear and distinct compositional separation.

5.3.4.3. Saturation magnetizations for the co-evaporated Co-Ag films. Figure 49 shows the $M_{\mathrm{s}}$ values for the co-evaporated Co-Ag samples. It can be seen that the saturation magnetization of the Co-Ag films decreases almost linearly with increasing $\mathrm{Ag}$ content. In order to exclude a mere dilution effect, a correction is made for the non-magnetic volume under the assumption of a complete separation of $\mathrm{Co}$ and Ag. The resulting ferromagnetic film volume $V^{*}$ is related to the total film volume $V$ by: 


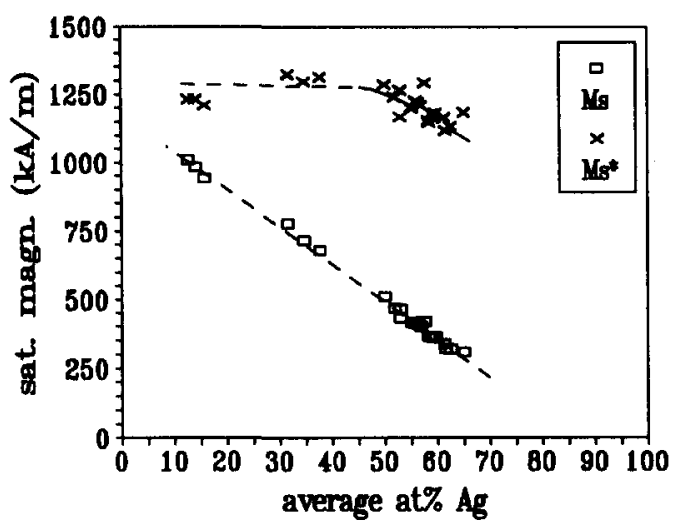

Fig. 49. Saturation magnetization as a function of the average $\mathrm{Ag}$ content for co-evaporated $\mathrm{Co}-\mathrm{Ag}$ films [1].

$$
\frac{V^{*}}{V}=\frac{\frac{4}{3} \pi r_{\mathrm{Co}}^{3} N_{\mathrm{Co}}}{\frac{4}{3} \pi r_{\mathrm{Co}}^{3} N_{\mathrm{Co}}+\frac{4}{3} \pi r_{\mathrm{Ag}}^{3} N_{\mathrm{Ag}}}
$$

In this expression the radii of the atoms are denoted as $r$ and the number of atoms as $N$, both with subscripts for $\mathrm{Ag}$ and $\mathrm{Co}$. The saturation magnetization under complete separation conditions, $M_{\mathrm{s}}^{*}$ can then be calculated by:

$M_{\mathrm{s}}^{*}=M_{\mathrm{s}} \frac{V^{*}}{V}=M_{\mathrm{s}}\left(1+\frac{\text { at. } \% \mathrm{Ag}}{\text { at. } \% \mathrm{Co}} \frac{r_{\mathrm{Ag}}{ }^{3}}{r_{\mathrm{Co}}{ }^{3}}\right)$

where $M_{\mathrm{s}}$ is the saturation magnetization if the total film volume would be ferromagnetic. Effectively this comes down to excluding the dilution effect (in clusters) that a second element, $\mathrm{Ag}$, is supposed to have on $\mathrm{Co}$. If the films consist of clusters of Co on one side and $\mathrm{Ag}$ on the other side of the columns, then this dilution is merely a volume effect and the $M_{\mathrm{s}}^{*}($ at.\% $\% \mathrm{Ag})$ should be constant. If $\mathrm{Ag}$ is, to some extent, also present within the Co matrix, then besides the volume effect it also reduces the number of $\mathrm{Co}-\mathrm{Co}$ neighbours. The exchange energy between neighbouring atoms and the Curie temperature of the film are thereby reduced. With a homogeneous distribution of the two elements, a dilution factor of 0.8 lowers the $T_{\mathrm{c}}$ to $293 \mathrm{~K}$ [124]. In that case $M_{\mathrm{s}}(\mathrm{at} . \% \mathrm{Ag})$ decreases more strongly than in the case of separation merely into clusters, and $M_{s}^{*}(\mathrm{at} . \% \mathrm{Ag})$ remains a decreasing function. In the above expressions we neglected the volume of open regions. This volume part should be added to the total volume part. However, because no accurate numbers are known in our case, we decided to disregard them. Hence, the calculated $M_{\mathrm{s}}^{*}$ is underestimated, and with decreasing atomic percentage of $\mathrm{Co}$ the error incurred by this step becomes larger.

The calculated saturation magnetization $M_{s}^{*}$ is also given in Fig. 49. At low Ag content the $M_{\mathrm{s}}^{*}$ is almost constant with an increasing amount of Ag. This indicates that in the low $\mathrm{Ag}$ content region the $M_{\mathrm{s}}(\mathrm{Ag})$ is predominantly decreased by a mere dilution effect of $\mathrm{Ag}$ $\mathrm{Ag}$ clusters. The fact that the extrapolated value of $M_{\mathrm{s}}$ is not yet zero at 80 at.\% $\mathrm{Ag}$ (and thus not only a decrease of exchange moment occurs owing to fewer Co-Co neighbours) supports this. Thus $\mathrm{Co}$ and $\mathrm{Ag}$ have been separated and compositional separation has 
occurred in those samples co-evaporated at room temperature. In the high Ag content region, $M_{\mathrm{s}}^{*}$; decreases with an increasing amount of $\mathrm{Ag}$, as does $M_{\mathrm{s}}$. The decrease of $M_{\mathrm{s}}^{*}$ with average $\mathrm{Ag}$ content tends to be less than is expected for a homogeneous dilution effect (the slope through the data points is less steep).

Another possible reason for the weaker decrease of $M_{\mathrm{s}}(\% \mathrm{Ag})$ is an electron sharing of Co and Ag. Possibly an "electron transfer" of Co to $\mathrm{Ag}$ occurs, since $\mathrm{Ag}$ is the more noble metal of the two. A similar polarization mechanism takes place in $\mathrm{Co} / \mathrm{Pd}$ multilayers [125]. However, the electron transfer from $\mathrm{Co}$ to $\mathrm{Ag}$ is less likely than the occurrence of Co-Co clusters, and if it should take place, then it is probably a minor effect. Furthermore, the presence of Co-rich regions is proved by NMR measurements.

$\mathrm{Co}_{40} \mathrm{Ag}_{60}$ films deposited at a higher substrate temperature have slightly larger saturation magnetizations. The additional energy (larger surface diffusion) is apparently used to increase the separation of $\mathrm{Co}$ and Ag. Based on Miedema's model of the alloying behaviour of metals [126], this can be expected. With opposing vapour beams of Co and Ag under low surface-diffusion conditions, some mixing of $\mathrm{Co}$ and $\mathrm{Ag}$ will obviously occur, since there is no straight and strict borderline with respect to possible positions for impinging $\mathrm{Co}$ and $\mathrm{Ag}$ atoms, and, since $\mathrm{Ag}$ and $\mathrm{Co}$ are non-alloying metals, an increase in surface diffusion will allow further separation.

Also with successive annealing steps at 225,325 and $425^{\circ} \mathrm{C}$, each of two and a half hours, an increase in $M_{s}$ is found for $\mathrm{Co}_{40} \mathrm{Ag}_{60}$. The $\mathrm{Ag}$ that was present in the Co-rich regions can, with the additional energy supplied by the heat treatment, diffuse to the Agrich clusters, whereby the number of Co-Co neighbours is increased. NMR measurements proved that a much more compositionally separated state exists after annealing with only almost-pure Co regions. Hence, the increase in $M_{s}$ is caused by more compositional separation. The same annealing steps in a $\mathrm{Co}_{60} \mathrm{Ag}_{40}$ sample had almost no effect on $M_{\mathrm{s}}$. Possibly, oxidation of the larger Co content cancelled a presumable increment of $M_{\mathrm{s}}$ due to more compositional separation.

Summing up for Co-Ag: the separation of $\mathrm{Co}$ and $\mathrm{Ag}$ is both in $\mathrm{Co}-\mathrm{Co}$ and $\mathrm{Ag}-\mathrm{Ag}$ clusters (at low percentages of $\mathrm{Cr}, M_{\mathrm{s}}(\% \mathrm{Cr})$ decreases according to a "nearly-merely" volume-dilution, and $M_{\mathrm{s}}^{*}(\% \mathrm{Cr})$ is nearly constant and $M_{\mathrm{s}}$ is not yet zero at $\left.80 \mathrm{at} . \% \mathrm{Ag}\right)$, and $\mathrm{Ag}$ is also present in the Co matrix, thereby reducing the number of Co-Co neighbours. This is supported by NMR measurements. Furthermore, the Co-Ag samples deposited at an elevated substrate temperature $\left(T_{\mathrm{H}}\right)$ have higher saturation magnetizations than the lowtemperature samples, and the $M_{\mathrm{s}}$ increases after annealing. Since Co and $\mathrm{Ag}$ are nonalloying metals, the separation between them is expected to increase if additional energy is applied to the film. The Ag that was present in the Co matrix can then diffuse to the $\mathrm{Ag}$-rich clusters, whereby the number of Co-Co neighbours is increased. NMR measurements also support this, since after annealing only the resonance peak around $220 \mathrm{MHz}$ is present, implying that the Co is present as highly pure Co.

\subsubsection{Texture}

Next a brief comment on the (role of) texture in the films is given. In the case of the $\mathrm{Co}-\mathrm{Ag}$ and $\mathrm{Co}-\mathrm{Cr}$, the process-induced compositional separation and the oblique columnar morphology are related to the phase configuration and texture. In $\mathrm{Co}-\mathrm{Ag}$ two separate lattices are present. With more than 31 at.\% $\mathrm{Ag}$, fcc $\mathrm{Co}$ and fcc $\mathrm{Ag}$ lattices are present, both with the (111) planes approximately parallel to the film surface; with $13-16$ at.\% Ag the Co: lattice is hcp. The lattice parameters of the fcc $\mathrm{Ag}$ and fcc Co lattices are smaller 
and larger, respectively, than for the pure metals. With higher substrate temperatures they get closer to the pure metal lattice parameters. Distinct compositional separation, with regions existing of $\mathrm{Co}-\mathrm{Co}$ clusters and $\mathrm{Ag}-\mathrm{Ag}$ clusters, but also $\mathrm{Co}-$-rich regions with an amount of Ag in them, have an affinity with the phase configuration described above. After annealing, an hcp Co structure is present. The more highly pure Co regions that exist after the heat treatment can then develop an hcp lattice.

The Co-Cr samples consist of an hcp polycrystalline structure $[2-4,6]$. No separate Co and $\mathrm{Cr}$ lattices could be measured, since the (110) reflection of $\mathrm{Cr}$ overlaps the $\mathrm{Co}(0002)$ reflection. As with $\mathrm{Co}-\mathrm{Ag}$, some of the $\mathrm{Cr}$ is also present in the Co-rich regions which can be deduced from the increase of the lattice parameter with increasing $\mathrm{Cr}$ content. The hcp structure develops preferentially with the $c$ axis perpendicular to the substrate $[6,127]$. However, the oblique-incidence Co vapour with low adatom diffusion makes it very difficult for the adatoms to arrange themselves in a perpendicularly oriented (0002) texture. In lowtemperature evaporation the inclined columnar morphology is therefore accompanied by an inclined texture, with the fibre axis (which is the $c$ axis in hcp) oriented closer to the film normal than the columnar axis is. In high-temperature evaporation the $c$ axis is more perpendicular than it is in the samples where the Co vapour impinges perpendicularly.

\subsubsection{Consequences for the magnetic properties}

As with obliquely deposited single-source samples, the obliquely co-evaporated samples are also magnetically anisotropic in-plane. There is an in-plane anisotropy for the investigated co-evaporated samples of Table 1 (excluding the rotated ones) which is perpendicular to the incidence plane. The resulting in-plane anisotropy has several contributors.

First of all there is the "projection" of the tilted out-of-plane columnar-shape and crystalline anisotropies. The discontinuities formed by the open regions between the columns (due to the shadowing mechanism) and the $\mathrm{Cr}$ enhancement at the columnar boundaries (due to process-induced compositional separation) give rise to a columnar-shape anisotropy oriented along the columnar axis. The hcp crystalline structure causes a magnetocrystalline anisotropy, oriented along the $c$ axis. Both lie in the evaporation plane, and for the samples of the $M$ series, for instance, they are inclined with respect to the film normal: thus an in-plane anisotropy energy results, which is in the longitudinal in-plane direction (similar to that described in Section 4.8). In the rotated samples (LR series) the out-of-plane anisotropy is parallel to the film normal, and consequently makes no in-plane contribution.

Secondly, the difference between columnar and fibre-axis tilts gives rise to stress in the films and to a large number of stacking faults, by which the stress can, to some extent, be relieved. (The number of stacking faults is expected to be smallest in the rotated samples, since these samples have their $c$ axis parallel to the columnar axis.) The resulting stress is tensile and makes an in-plane contribution to the anisotropy.

Thirdly, the discontinuities in the longitudinal direction (open regions and $\mathrm{Cr}$ enhancement at columnar boundaries) also contribute to an in-plane anisotropy, which is perpendicular to the evaporation plane (transverse direction). This shape anisotropy is even more enhanced if clustering of columns occurs, such as with the so-called bundles. It increases with film thickness, presumably because the columnar dimensions become (slightly) larger and/or because the compositionally separated state becomes more inhomogeneous (measurement result of NMR). It is less strong in the films with elongated columns in the longitudinal direction. The in-plane anisotropies and the discontinuities lead to non-isotropic in-plane behaviour, and consequently the in-plane coercivities differ in the longitudinal and transverse 
directions. The rotated samples are in-plane isotropic, obviously because the rotation averages out any anisotropic effects. In contrast to the co-evaporated samples, the effective in-plane direction for our single-source obliquely evaporated samples ( $S$ series) is parallel to the incidence plane. This is due to the small transverse anisotropy constant, which is $4 \mathrm{~kJ} \mathrm{~m}^{-3}$ or less, corresponding to the fact that no bundles are observed and no $\mathrm{Cr}$ enrichment at the columnar boundaries is present.

The different behaviour found on annealing for the $\mathrm{Co}-\mathrm{Cr}$ samples of the different series is striking. The compositionally separated state is seen as the dominant parameter for this. No significant changes in morphology were observed.

$M-T_{R}$ sample. Possible change from process-induced compositional separation to thermally-enhanced compositional separation and possibly more oxidation after $400{ }^{\circ} \mathrm{C}$ annealing, resulting in very small increases in $M_{\mathrm{s}}$ and $H_{\mathrm{c} \perp}$, see Fig. 50 . Since in the as-deposited state $\mathrm{Cr}$ is, in the majority, only present at one side of the columns, the possibility of rearrangement of $\mathrm{Cr}$ (diffusion within the columns) is limited.

$L R-T_{R}$ sample. Although for this sample the compositionally separated state is more homogeneous than for the $\mathrm{M}-T_{\mathrm{R}}$ sample, the process geometry is believed to have resulted in a small amount of $\mathrm{Cr}$ enrichment at all sides of the column boundaries. Rearrangement of $\mathrm{Cr}$, in the form of diffusion into the columns, is thus easier than for the $\mathrm{M}-T_{\mathrm{R}}$ sample. A microstructural change is also indicated by the decreasing lattice spacing of the $\{0002\}$ planes (from $2.030 \AA$ to $2.028 \AA$ ). The anneal step of $250^{\circ} \mathrm{C}$ is already sufficient to cause a homogenization $\left(M_{s}\right.$ and the tilted out-of-plane anisotropy constant, $K_{e}$, decrease drastically; $K_{\mathrm{e}}$ decreases from 116 to $68 \mathrm{~kJ} \mathrm{~m}^{-3}$ ). Annealing at $400{ }^{\circ} \mathrm{C}$ probably increases oxidation effects, whereby $M_{\mathrm{s}}, H_{\mathrm{c} \perp}$ and $K_{\mathrm{e}}$ increase (less exchange due to non-ferromagnetic oxides; $K_{\mathrm{e}}$ increases to $135 \mathrm{~kJ} \mathrm{~m}^{-3}$ ).

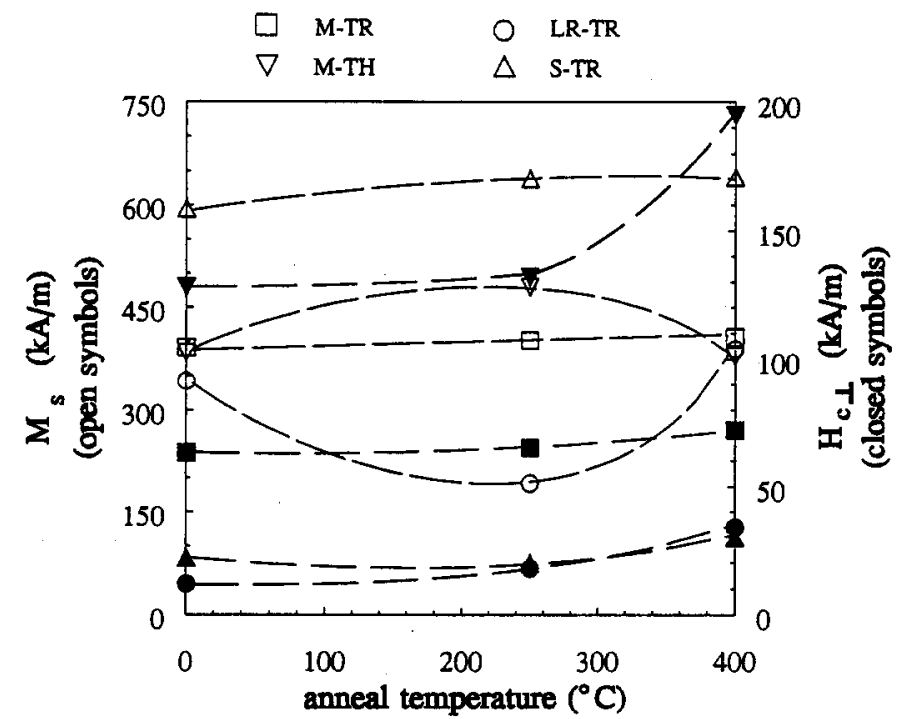

Fig. 50. Saturation magnetization and perpendicular coercivity as a function of the annealing temperature for samples of the $M, L R$ and $S$ series [1]. 
$S-T_{R}$ sample. Only small changes occur. The as-deposited sample is in a relatively homogeneous state. Annealing increases thermally enhanced compositional separation and/ or oxidation. Both effects lead to the observed increases in $M_{\mathrm{s}}$ and $H_{\mathrm{c} \perp}$.

$M-T_{H}$ sample. The as-deposited sample is in a compositionally separated state, induced by the preheating of the substrate and possibly still some effect of the opposing vapour directions. The measured substrate temperature at the back of the Si substrate was below $150{ }^{\circ} \mathrm{C}$ after preheating occurred. This temperature is below the temperature where the maximum of thermally enhanced compositional separation normally occurs (see, for example, ref. 119). The anneal step at $250^{\circ} \mathrm{C}$ can therefore increase the compositionally separated state even further, whereby $M_{\mathrm{s}}, H_{\mathrm{c} \perp}$ and $K_{\mathrm{e}}$ increase $\left(K_{\mathrm{e}}\right.$ increases from 147 to $218 \mathrm{~kJ}$ $\mathrm{m}^{-3}$ ). The anneal step at $400{ }^{\circ} \mathrm{C}$ causes homogenization and oxidation $\left(M_{\mathrm{s}}\right.$ decreases and $H_{\mathrm{c} \perp}$ increases further, see Fig. $50 ; K_{\mathrm{e}}$ decreases to $\left.180 \mathrm{~kJ} \mathrm{~m}^{-3}\right)$. Also in this sample a microstructural change is supported by the decreasing lattice spacing of the $\{0002\}$ planes upon annealing (from $2.036 \AA$ to $2.030 \AA$; similar to results from the literature (for example, ref. 128).

The change in the transverse anisotropy constants for both samples of the $M$ series is attributed to changes in column aggregation and shape, similar (but smaller) to singlesource obliquely evaporated $\mathrm{Co}-\mathrm{Cr}$ from the literature [129]. In our films, especially the rearrangement of the ferromagnetic parts will contribute.

In conclusion: using the oblique-incidence vapour flux the morphology, texture and also the anisotropy can be tailored. Applying ferromagnetic $\mathrm{Co}$ and a non-ferromagnetic metal in opposing directions the distribution of the two metals can be manipulated. If a suitable metal, such as $\mathrm{Cr}$, is chosen for the non-ferromagnetic element, then an enhanced energy product can be developed, with deposition on substrates already at ambient temperature. However, rotation of the substrate during evaporation does not yield good properties. If the non-ferromagnetic element destroys the hcp lattice; as was the case with $\mathrm{Ag}$ and $\mathrm{Ta}$, then the anisotropy is no longer strong enough to overcome the film demagnetization, and the medium loses its potential for high-density recording.

\subsection{Summary}

In Section 5 it has become clear, from the various examples given of thin films for magnetic recording applications, that anisotropic magnetic properties can be tailored by means of anisotropic morphological characteristics. These features are due to different packing densities in the longitudinal and transverse in-plane directions. With respect to the magnetic properties, the packing density of the ferromagnetic units is of importance. Generally speaking, the anisotropic morphology can be exploited for other applications as well. For instance, the electrical resistivity and optical reflectivity depend on the material packing.

By using two oblique vapour directions instead of one, an additional parameter is provided to tailor the microstructure of the film. As compared to the single-source situation, the following important differences exist. With respect to the morphology, the degree of columnar tilting is manipulated by the two incoming vapour fluxes. Further different shapes of crystallites can arise. In the low-mobility region of the investigated films, for example, elongated shapes which have their long axes parallel to the incidence plane can more easily arise than with one oblique vapour flux. In Section 5.2 examples of the morphology were discussed. Besides the above-mentioned differences, also features that the co-evaporation and single-source deposition have in common were discussed, such as columnar structure 
and bundling. The discussion included the effects of the parameters, such as the substrate temperature, that are normally used to tailor the microstructure.

Another important aspect that can be manipulated by using two oblique vapour directions is the local chemical distribution in the lateral direction. In our case of thin films for magnetic recording applications, we have shown in Section 5.3 that, by using oblique and opposing ferromagnetic and non-ferromagnetic vapour fluxes, a chemically inhomogeneous distribution can be induced in the lateral direction under the condition of low adatom mobility. We call this effect process-induced compositional separation. The existence of alternating ferromagnetic and non-ferromagnetic regions is advantageous, because it decreases the magnetostatic exchange energy locally and provides pinning points for domain walls. Process-induced compositional separation thus allows deposition at low substrate temperatures of films that have an enhanced energy product and a reversal of the magnetization that is most likely to proceed through either rotation or domain-wall movement where displacement of the domain walls is largely hindered by both the open regions between the columns and the chemical inhomogeneities (i.e. the non-ferromagnetic parts that are concentrated at one side of the columns).

Process-induced compositional separation can, by the choice of a suitable material, also be used in applications other than magnetic recording. Instead of choosing a ferromagnetic and a non-ferromagnetic metal, for instance, a high-conductivity and a low-conductivity material can be used. Anisotropic electrical properties that originate from an anisotropic morphology can thus be enhanced even further.

Even though in Section 5 we have mainly discussed thin films for (perpendicular) magnetic recording applications, the underlying mechanisms of the growth of the films and the influence they have on the magnetic properties are of greater importance than just for magnetic media alone. Because the anisotropic magnetic properties are strongly related to anisotropic morphological features, the exploitation of one and two (or more) oblique vapour directions can also be used for inducing anisotropic features for other than magnetic applications, such as optical applications and in connection with (electrical) conduction.

\section{Conclusions}

Based on both experimental data and simulations of thin-film growth, two major conclusions can be drawn from this paper. The simulations have proved to be a useful tool to predict the growth and support the interpretation of the experimental data. The two conclusions are:

1. Oblique-incidence deposition provides a tool to tailor the morphology. First of all, a columnar structure develops that is inclined, with the inclination angle depending on the incidence angle and the adatom mobility. Further different morphologies arise in the incidence plane (open regions between the columns in longitudinal direction) and in the transverse plane (dense packing or even aggregation - "bundles" - in the transverse direction). Since the morphology is related to physical parameters, such as electrical resistance, absorptional coefficients, magnetic-shape anisotropy and magnetic coercivity, its specific features can be exploited to realize anisotropic behaviour in obliquelydeposited thin films. The size, inclination and separation of the columns are important; for instance, in the example of magnetic thin films they can result in a magnetic-shape anisotropy along the columnar axes and an in-plane shape anisotropy in the transverse 
direction. Furthermore, different in-plane coercivities in the longitudinal and transverse directions can arise.

By choosing evaporation or ion-beam sputtering, the incidence angle can be satisfactorily controlled. However, this is not easy for the adatom mobility, since it depends on several deposition parameters, as discussed in Section 4.2. Furthermore, another critical point about tailoring the morphology is the packing density in the transverse direction. The (non)-appearance of bundles is sensitive to many deposition parameters (as became clear from the examples in Section 4.6), and an optimum is likely to exist. The tailoring parameter in the incidence plane, being the incidence angle, is in this context a much stronger tool than the optimum of all the other deposition parameters which together form the tailoring tools in the transverse direction. Non-bundling may be most easily attained under conditions of higher adatom mobility, since in this case the density of the nuclei is less and their dimensions and separation are larger. The most favourable conditions for an increased separation between columnar units are thus given by a higher ratio of substrate and melting temperatures, a lower deposition rate, lower partial pressures and higher incidence angles.

2. Oblique co-deposition provides an additional tool, under low-energy conditions, to tailor the local composition. By using opposing directions of materials with different characteristics, the distribution of the materials used in the film yields a different behaviour locally. The manipulation of the distribution of the materials used is done by making use of the shadowing effect during growth of the thin film. Thus, once again the incidence angles and adatom mobility are the crucial parameters for controlling the structure. In Section 5 we showed examples of the manipulation of two metals, using opposing directions of ferromagnetic Co and a non-ferromagnetic metal. We called this effect process-induced compositional separation. Here the existence of alternating ferromagnetic and nonferromagnetic regions was advantageous for magnetic-recording applications. The possibility to induce such regions without an increased substrate temperature enables the use of the relatively cheaper polyester substrates.

Besides the chemically inhomogeneous distribution in the lateral direction, the second oblique vapour direction also influences the morphology. In Section 5 we also discussed differences and similarities between the single- and dual-source geometries. These included the degree of columnar tilting, the shape of the crystallites and bundling (see the summary section 5.4).

\section{References}

1 H. van Kranenburg, Obliquely co-evaporated thin films for magnetic recording, Ph.D. Thesis, Twente University, Enschede, 1992, ISBN 90-9005476-6.

2 H. van Kranenburg, J.C. Lodder, Y. Maeda, L. Toth and Th.J.A. Popma, IEEE Trans. Magn., MAG-26 (1990) 1620.

3 H. van Kranenburg, J.C. Lodder and Th.J.A. Popma, J. Magn. Magn. Mat., 120 (1993) 225.

4 H. van Kranenburg, J.C. Lodder, Th.J.A. Popma, K. Takei and Y. Maeda, J. Magn. Soc. Jpn., 15, Suppl. No. S2 (1991) 33.

5 H. van Kranenburg, J.C. Lodder and Th.J.A. Popma, J. Magn. Magn. Mat., 120 (1993) 353.

6 S. Müller-Pfeiffer, H. van Kranenburg and J.C. Lodder, Thin Solid Films, 213 (1992) 143.

7 L. Holland, J. Opt. Sci. Am., 43 (1953) 376.

8 M. Kamiya, K. Hara and H. Fujiwara, J. Phys. Soc. Jpn., 47 (1979) 1371.

9 M.S. Cohen, J. Appl. Phys., 32 (1961) 87S.

10 D.O. Smith, M.S. Cohen and G.P. Weiss, J. Appl. Phys., 31 (1960) 1755. 
11 D.O. Smith, J. Appl. Phys., 30 (1959) 264 S.

12 K. Kuwahara and J. Hirota, Jpn. J. Appl. Phys., 13 (1974) 1093.

13 E.W. Pugh, E.L. Boyd and J.F. Freedman, IBM J., 4 (1960) 163.

14 E.M. Rossi, G. McDonough, A. Tietze, T. Arnoldussen, A. Brunsch, S. Doss, M. Henneberg, F. Lin, R. Lyn, A. Ting and G. Trippel, J. Appl. Phys., 55 (1984) 2254; T.C. Arnoldussen, E.M. Rossi, A. Ting, A. Brunsch, J. Schneider and G. Trippel, IEEE Trans. Magn., MAG-20 (1984) 821.

15 T. Motohiro and Y. Taga, Appl. Opt., 28 (1989) 2466.

16 M. Suzuki and Y. Taga, J. Appl. Phys., 71 (1992) 2848.

17 K.L. Chopra, Thin Film Phenomena, McGraw-Hill, New York, 1969, p. 27.

18 M. Klaua, in H. Bith and J. Heidenreich (eds.), Electron Microscopy in Solid State Physics, Materials Science Monographs, 40, Elsevier, Amsterdam, 1987, p. 454.

19 J.V. Sanders, in J.R. Anderson (ed.), Chemisorption and Reaction on Metallic Films, Academic Press, London and New York, 1971, p. 1.

20 P.B. Barna, in L. Eckertova and T. Ruzicka (eds.), Diagnostics and Applications of Thin Films, IOP Publishing, Bristol, UK, 1992, p. 295.

21 Y. Arima and T. Irisawa, J. Cryst. Growth, 115 (1991) 428.

22 A.I. Vovsi, L.P. Strakhov and O.A. Yakovuk, Sov. Phys. - Solid State, 14 (1972) 1251.

23 B.A. Movchan and A.V. Demchishin, Fiz. Metal. Metalloved., 28 (1969) 653.

24 J.A. Thornton, J. Vac. Sci. Technol., 11 (1974) 666.

25 J.A. Thornton, J. Vac. Sci. Technol., 12 (1975) 830.

26 C.R.M. Grovenor, H.T.G. Hentzell and D.A. Smith, Acta Metall., 32 (1984) 773.

27 R. Messier, A.P. Giri and R.A. Roy, J. Vac. Sci. Technol. A, 2 (1984) 500.

28 B.G. Demczyk, J. Magn. Mat., 102 (1991) 238

29 B. Messier and J.E. Yehoda, J. Appl. Phys., 58 (1985) 3739.

30 J.E. Yehoda and R. Messier, Appl. Surf. Sci., 22/23 (1985) 590.

31 R. Messier, J. Vac. Sci. Technol. A, 4 (1986) 490.

32 A. van der Drift, Philips Res. Rep., 22 (1967) 267.

33 R. Hergt and H. Pfeiffer, Phys. Stat. Sol. (a), 92 (1985) K89.

34 G.S. Bales and A. Zangwill, J. Vac. Sci. Technol. A, 9 (1991) 145.

35 A.G. Dirks, R.A.M. Wolters and A.E.M. De Veirman, Thin Solid Films, 208 (1992) 181.

36 H. König and G. Helwig, Optik, 6 (1950) 111 (in German).

37 A.G. Dirks and H.J. Leamy, Thin Solid Films, 47 (1977) 219.

38 N.G. Nakhodkin and A.I. Shaldervan, Thin Solid Films, 10 (1972) 109.

39 J.G.W. van de Waterbeemd and G.W. van Oosterhout, Philips Res. Rep., 22 (1967) 375.

40 V. Kambersky, Z. Málek, Z. Frait and M. Ondris, Czech. J. Phys. B, 11 (1961) 171.

41 M. Hashimoto, Thin Solid Films, 115 (1984) 309.

42 B. Lewis and J.C. Anderson, Nucleation and Growth of Thin Films, Academic Press, New York, 1978, Chapter 3.IV.

43 D.K. Pandya, A.C. Rastogi and K.L. Chopra, J. Appl. Phys., 46 (1975) 2966.

44 K. Yoshida, K. Imagawa, Y. Honda, M. Futamoto and H. Daimon, Jpn. J. Appl. Phys., 27 (1988) 1240.

45 L. Holland, Vacuum Deposition of Thin Films, Chapman and Hall, London, 1963, Chapter 5.

46 L. Bangjun and H.A. Macleod, SPIE Southwest Conference on Optics, 540 (1985) 150.

47 D. Henderson, M.H. Brodsky and P. Chaudhary, Appl. Phys. Lett., 25 (1974) 641.

48 K.H. Müller, Phys. Rev. B, 35 (1987) 7906.

49 S. Müller-Pfeiffer, H.J. Anklam and W. Raubenreisser, Phys. Stat. Sol. (b), 160 (1990) 491.

50 J.M. Nieuwenhuizen and H.B. Haanstra, Philips technisch tijdschrif, 27 (1965/66) 115 (in Dutch); also in Philips Tech. Rev., 27 (1966) 87.

51 H. Fujiwara, K. Hara, M. Kamiya, T. Hashimoto and K. Okamoto, Thin Solid Films, 163 (1988) 387.

52 H.J. Leamy and A.G. Dirks, J. Appl. Phys., 49 (1978) 3430.

53 P. Meakin, P. Ramanlal, L.M. Sander and R.C. Ball, Phys. Rev. A, 34 (1986) 5091.

54 K. Okamoto, T. Hashimoto, K. Hara, M. Kamiya and H. Fujiwara, Thin Solid Films, 129 (1985) 299.

55 K. Okamoto, K. Hara, M. Kamiya, T. Hashimoto and H. Fujiwara, Thin Solid Films, 176 (1989) 255.

56 M. Kamiya, K. Hara, T. Hashimoto, K. Okamoto and H. Fujiwara, J. Phys. Soc. Jpn., 52 (1983) 3583.

57 K. Hara, M. Kamiya, T. Hashimoto, K. Okamoto and H. Fujiwara, Thin Solid Films, 158 (1988) 239.

58 K. Okamoto, T. Hashimoto, H. Fujiwara, K. Hara and M. Kamiya, J. Magn. Mat., 81 (1989) 374.

59 Y. Takeno and Y. Iwama, J. Magn. Mat., 35 (1983) 293.

60 E.I. Kondorsky and P.P Denisov, IEEE Trans. Magn., MAG-6 (1970) 167.

61 K. Hara, T. Kamimori, H. Fujiwara and T. Hashimoto, Thin Solid Films, 66 (1980) 185.

62 T. Hashimoto, K. Okamoto, H. Fujiwara, K. Itob, K. Hara and M. Kamiya, Thin Solid Films, 192 (1990) 335.

63 S. Nakahara, K. Kuwahara and A. Nishimura, Thin Solid Films, 72 (1980) 297.

64 E. Münster and J. Richter-Mendau, Experimentelle Technik der Physik XXIV, Heft 1 (1976) 51 (in German). 
65 K. Hara, K. Itoh, M. Kamiya, K. Okamoto, T. Hashimoto and H. Fujiwara, J. Magn. Magn. Mat., 102 (1991) 247.

66 T. Tymosz, K. Swiderczak and K. Branska, Phys. Stat. Sol. (a), 118 (1990) 519.

67 N.G. Dhere, R.G. Pinheiro and N.R. Parikh, J. Vac. Sci. Technol., 11 (1974) 599.

68 A.G. Dirks and H.J. Leamy, IEEE Trans. Magn., MAG-14 (1978) 835.

69 E. Bauer, in M.H. Francombe and H. Sato (eds.), Single-Crystal Films, Pergamon Press, Oxford, 1964, p. 43.

70 K. Okamoto, T. Hashimoto, K. Hara, M. Kamiya and H. Fujiwara, Thin Solid Films, 147 (1987) 299.

71 K. Itoh, J. Magn. Magn. Mat., 95 (1991) 237.

72 K. Okamoto, K. Itoh and T. Hashimoto, J. Magn. Magn. Mat., 87 (1990) 379.

73 E.I. Kondorsky and P.P. Denisov, IEEE Trans. Magn., MAG-6 (1970) 167.

74 K. Ozawa, T. Yanada, H. Masuya, M. Sato, S. Ishio and M. Takahashi, J. Magn. Magn. Mat., 35 (1983) 289.

75 J.M. Alameda, M. Torres and F. López, J. Magn. Magn. Mat., 62 (1986) 209.

76 R. Sugita, IEEE Trans. Magn., MAG-20 (1984) 687.

77 R. Sugita, T. Nambu and Y. Sakamoto, IEEE Trans. Magn., MAG-23 (1987) 2449.

78 H. Hibst, in K.H.J. Buschow, G.J. Long and F. Grandjean (eds.), High Density Digital Recording, Kluwer Academic Press, 1993, Chapter 5.

79 S. Keitoku, S. Negishi, I. Tsuchitori and M. Goto, IEEE Transl. J. Magn. Jpn., TJMJ-5 (1990) 1114.

80 H.J. Leamy and A.G. Dirks, J. Phys. D: Appl. Phys., 10 (1977) L95.

81 J.G.W. van de Waterbeemd and G.W. van Oosterhout, Philips Res. Rep., 22 (1967) 375.

82 R. Harman, J. Liday and M. Veselý, Proc. 2nd Int. Conf. on Solid Surfaces 1974, Jpn. J. Appl. Phys., Suppl. 2(2) (1974) 823.

83 J.E. Snyder and M.H. Kryder, Phys. Rev. (1993), in press.

84 S. Keitoku and K. Nishioka, Jpn. J. Appl. Phys., 20 (1981) 1249.

85 J. Kim, S.H. Wen and D. Yee, J. Vac. Sci. Technol. A, 6 (1988) 2366.

86 F.A. Pronk and J.C. Lodder, IEEE Trans. Magn., MAG-24 (1988) 1744.

87 F.A. Pronk and J.C. Lodder, J. Phys. C, 8, Suppl. No. 12 (1988) 1991.

88 M. Atzmon, D.A. Kessler and D.J. Srolovitz, J. Appl. Phys., 72 (1992) 442.

89 M. Hasebe, K. Oikawa and T. Nishizawa, J. Jpn. Inst. Met., 46 (1982) 577 (in Japanese).

90 K. Ishida and T. Nishizawa, Bull. Alloy Phase Diagrams, 11 (1990) 357.

91 J.E. Snyder and M.H. Kryder, J. Appl. Phys., 73 (1993) 5551.

92 A.R. Miedema, Philips Tech. Rev., 33 (1973) 149.

93 S. Keitoku, T. Kamimori and M. Goto, Jpn. J. Appl. Phys., 25 (1986) 1668.

94 S. Keitoku, M. Goto and T. Kamimori, in M. Takahashi, S. Maekawa, Y. Gondō and H. Nosé (eds.), Proc. Int. Symp. Physics of Magnetic Materials, Sendai, Japan, 8-11 April 1987, World Scientific, Singapore, 1987, p. 101.

95 M. Suzuki and Y. Taga, J. Appl. Phys., 71 (1992) 2848.

96 Y. Takeda, T. Motohiro and S. Noda, in M. Doyama, T. Suzuki, J. Kihara and R. Yamamoto (eds.), Computer Aided Innovation of New Materials, North-Holland, Amsterdam, 1991, p. 965.

97 T. Motohiro, Y. Takeda, Y. Watanabe and S. Noda, in R.S. Averback, J. Bernholc and D.L. Nelson (eds.), Clusters and cluster assembled materials, Mat. Res. Soc. Symp. Proc., 206 (1991) 423.

98 H.J. Leamy and A.G. Dirks, J. Appl. Phys., 50 (1979) 2871.

99 Y. Maeda and M. Asahi, J. Appl. Phys., 61 (1987) 1972.

100 J.N. Chapman, I.R. McFadyen and J.P.C. Bernards, J. Magn. Magn. Mat., 62 (1986) 359.

101 D.J. Rogers, J.N. Chapman, J.P.C. Bernards and S.B. Luitjens, IEEE Trans. Magn., MAG-25 (1989) 4180.

102 K. Hono, Y. Maeda, J-L. Li and T. Sakurai, J. Magn. Magn. Mat., 110 (1992)

103 K. Hono, S.S. Babu, Y. Maeda, N. Hasegawa and T. Sakurai, Appl. Phys. Lett., 62 (1993) 2504.

104 K. Takei and Y. Maeda, Jpn. J. Appl. Phys., 30 (1991) L1125.

105 K. Yoshida, H. Kakibayashi and H. Yasuoka, J. Appl. Phys., 68 (1990) 705.

106 J.C. Lodder, H. van Kranenburg, K. Takei and Y. Maeda, J. Magn. Magn. Mat., 118 (1993) 248.

107 F.A. Pronk and J.C. Lodder, IEEE Trans. Magn., MAG-24 (1988) 1744.

108 S. Honda and J. Storer, IEEE Trans. Magn., MAG-22 (1986) 337.

109 R. Sugita and F. Kobayashi, IEEE Trans. Magn., MAG-18 (1982) 1818.

110 K. Ouchi and S. Iwasaki, IEEE Trans. Magn., MAG-23 (1987) 2443.

111 S. Nakagawa, Y. Furuto and M. Naoe, J. Magn. Magn. Mat., 104-107 (1992) 1801.

112 F. Bolzoni, F. Leccabue, R. Panizzieri and L. Pareti, J. Magn. Magn. Mat., 31-34 (1983) 845.

113 K. Yoshida, H. Kakibayashi and H. Yasuoka, Mat. Res. Soc. Symp. Proc., 232 (1991) 47.

114 N. Zhang and J.C. Lodder, J. Magn. Magn. Mat., 89 (1990) 284.

115 W.G. Haines, J. Appl. Phys., 55 (1984) 2263.

116 A. Fartash and H. Oesterreicher, J. Appl. Phys., 66 (1989) 3275.

117 W.J.M.A. Geerts, J.G.Th. te Lintelo, J.C. Lodder and Th.J.A. Popma, IEEE Trans. Magn., MAG-26 (1990) 36.

118 A. Werner, H. Hibst and H. Mannsperger, IEEE Trans. Magn., MAG-26 (1990) 103.

119 Y. Maeda and M. Asahi, IEEE Trans. Magn., MAG-23 (1987) 2061. 
120 M. Hansen, Constitution of Binary Alloys, McGraw-Hill, New York, 1958; R.P. Elliot, Constitution of Binary Alloys, Ist suppl., McGraw-Hill, New York, 1965.

121 K. Hayashi, M. Hayakawa, Y. Ochiai, H. Matsuda, W. Ishikawa and K. Aso, J. Appl. Phys., 61 (1987) 2983.

122 M. Naoe, M. Kodaira, Y. Hoshi and S. Yamanaka, IEEE Trans. Magn., MAG-17 (1981) 3062.

123 M. Naoe, H. Kazama, Y. Hoshi and S. Yamanaka, J. Appl. Phys., 53 (1982) 7846.

124 T. Wielinga, Investigations on perpendicular magnetic recording, Ph.D. Thesis, Twente University, Enschede, 1983, pp. 37-38.

125 P. de Haan, Q. Meng, T. Katayama and J.C. Lodder, J. Magn. Magn. Mat., 113 (1992) 29.

126 A.R. Miedema, Philips Tech. Rev., 33 (1973) 149.

127 K. Yoshida, K. Imagawa, Y. Honda, M. Futamoto and H. Daimon, Jpn. J. Appl. Phys., 27 (1988) 1240.

128 Y. Uchiyama, K. Ishibashi, H. Sato, T. Ueda and U.-M. Hwang, Jpn. J. Appl. Phys., 29 (1990) 1450.

129 T. Tymosz, K. Swiderczak and K. Branska, Phys. Stat. Sol. (a), 118 (1990) 519. 